Copyright

by

Paul John Huxel

2006 
The Dissertation Committee for Paul John Huxel

certifies that this is the approved version of the following dissertation:

\section{Navigation Algorithms and Observability Analysis for Formation Flying Missions}

Committee:

Robert H. Bishop, Supervisor

Maruthi R. Akella

David G. Hull

Cesar A. Ocampo

James R. Carpenter 


\title{
Navigation Algorithms and Observability Analysis for Formation Flying Missions
}

\author{
by
}

Paul John Huxel, B.S., M.S.

\author{
DISSERTATION \\ Presented to the Faculty of the Graduate School of \\ The University of Texas at Austin \\ in Partial Fulfillment \\ of the Requirements \\ for the Degree of \\ DOCTOR OF PHILOSOPHY
}

THE UNIVERSITY OF TEXAS AT AUSTIN

May 2006 
In Loving Memory Of

My Dad

\&

My Brother 


\section{Acknowledgments}

I wish to express my sincere gratitude to my advisor, Dr. Robert $\mathrm{H}$. Bishop, for his inspiration and guidance. His enthusiastic, yet patient tutelage has deepened my understanding and appreciation of spacecraft navigation. Thank you for the opportunities this experience has already provided, as well as those yet to come.

I would also like to extend this appreciation to my committee members, Dr. Maruthi R. Akella, Dr. David G. Hull, Dr. Cesar A. Ocampo, and Dr. James R. Carpenter of the NASA Goddard Space Flight Center for their assistance in the preparation of this dissertation.

I should also recognize Mike Moreau and the NASA Goddard Space Flight Center, as well as the UT Department of Aerospace Engineering and Engineering Mechanics, for their financial support.

My graduate experience at UT has benefited intellectually, as well as personally, from the camaraderie of my fourth floor "laboratory colleagues." In particular I wish to acknowledge Renato Zanetti, Kyle Demars, and Nick Lin.

I wish to thank all of my friends and family for their unwavering support throughout the years: The Meske family, Jay Anseth, Mike Ruiz, and Matt Porro just to name a few. And last, but certainly not least, I wish to extend 
my eternal gratefulness to those who have provided me with strength and determination: my Grandma, my sisters Michele, Suzanne, and Jackie, and above all, my Mom. 


\title{
Navigation Algorithms and Observability Analysis for Formation Flying Missions
}

\author{
Publication No. \\ Paul John Huxel, Ph.D. \\ The University of Texas at Austin, 2006
}

Supervisor: Robert H. Bishop

\begin{abstract}
Navigation algorithms and the corresponding observability analysis for formation flying missions are developed. The methodology of the observability analysis relates the physical geometry of the observers, as well as the spacecraft formation, to several measures of system observability. Relationships between these observability measures and the state error covariance are then derived to provided estimated bounds or forecasts for the expected navigation accuracy. These methods range from conservative time-invariant analytic bounds to more representative numerical forecasts using common dilution of precision metrics.

The research also examines the robustness of the extended Kalman filter when simultaneously processing inertial and relative range measurements. It has been shown that processing relative range measurements in conjunction with inertial range measurements can directly increase the accuracy of the inertial state estimate. However, it has also been shown that when there
\end{abstract}


is relatively large uncertainty in the state estimate the addition of relative measurements can cause an otherwise convergent filter to diverge. This dissertation considers several methods for preventing this divergence, as well as an in-depth examination of second-order terms to explain the basis of the problem. In particular, to illustrate their potential significance, analytical bounds are derived for the second-order terms.

viii 


\section{Table of Contents}

Acknowledgments $\quad$ v

Abstract vii

List of Tables $\quad$ xi

List of Figures $\quad$ xii

Chapter 1. Introduction 1

1.1 Review of Existing Literature . . . . . . . . . . . . . . 2

1.2 Research Contributions . . . . . . . . . . . . . . 8

1.3 Organization of the Dissertation . . . . . . . . . . . . 10

Chapter 2. Foundation of Benchmark Formation 11

2.1 Mathematical Notation and Parameters . . . . . . . . . . . . 11

2.2 Coordinate Systems and Frame Transformations . . . . . . . . 13

2.3 Lunar Benchmark Problem Formulation . . . . . . . . . . . . . 17

2.3.1 Mission and Formation Description . . . . . . . . . 18

2.3.2 System and Measurement Filter Models . . . . . . . . . 31

$\begin{array}{lll}\text { Chapter 3. Filter Algorithms } & 36\end{array}$

3.1 General Models . . . . . . . . . . . . . . . . . 37

3.2 Model Linearization . . . . . . . . . . . . . . . . . . . . 39

3.3 State and State Error Covariance Propagation . . . . . . . . . 43

3.4 State and State Error Covariance Update . . . . . . . . . . . . 44

3.5 Numerical Issues . . . . . . . . . . . . . . . . . . . . . . . . . . . . . . . . . . . . . . . . . . . . .

3.6 Algorithm Summary . . . . . . . . . . . . . . . . 48 
Chapter 4. Navigation Observability Analysis $\quad 50$

4.1 Observability Matrices and Measures . . . . . . . . . . 51

4.1 .1 Linear Time-Invariant . . . . . . . . . . . . . . . 51

4.1 .2 Nonlinear . . . . . . . . . . . . . . . . . . . . 55

4.1 .3 Linear Time-Varying . . . . . . . . . . . . . . 56

4.2 Observer Geometry Significance . . . . . . . . . . . . . 59

4.2 .1 Cluster-Observer Geometry . . . . . . . . . . . . 60

4.2 .2 Formation-Observer Geometry . . . . . . . . . . . . 69

4.2 .3 Constant Formation Geometry . . . . . . . . . . . 84

4.3 Observability-Error Covariance Relationship . . . . . . . . 93

4.3.1 LTI-Based Covariance Bound . . . . . . . . . . . . . 94

4.3.2 Cramér-Rao Covariance Bound . . . . . . . . . . . . 98

4.3.3 DOP-Based Covariance Forecast . . . . . . . . . . . . 109

4.4 Summary of Observability Simulation Results . . . . . . . . 113

Chapter 5. Relative Range Measurement Fusion 115

5.1 Fusion Complications . . . . . . . . . . . . . . . . . . 117

5.2 First-Order Robustness Enhancement Algorithms . . . . . . . 121

5.2 .1 Scalar Update Scheme . . . . . . . . . . . . . . . . 122

5.2.2 Process and Measurement Noise Schemes . . . . . . . 124

5.2 .3 Selective Measurement Scheme . . . . . . . . . . . . 127

5.3 Second-Order Robustness Enhancement Algorithms . . . . . . 128

5.4 Robustness Enhancement Simulation Results . . . . . . . . . . 141

$\begin{array}{lll}\text { Chapter 6. Conclusions } & 143\end{array}$

6.1 Research Summary . . . . . . . . . . . . . . . . . . . 143

6.2 Supplementary Research . . . . . . . . . . . . . . . . . . . 144

6.3 Future Research Considerations . . . . . . . . . . . . . . 146

$\begin{array}{ll}\text { Bibliography } & 149\end{array}$

$\begin{array}{ll}\text { Vita } & 157\end{array}$ 


\section{List of Tables}

2.1 Celestial Body Parameters . . . . . . . . . . . . . . . 13

2.2 Lunar Formation Parameters . . . . . . . . . . . . . . . . . . 22

2.3 Initial Libration Point Locations . . . . . . . . . . . . . . . . 26

3.1 Filter Algorithm Summary . . . . . . . . . . . . . . . . . 48

3.2 Second-Order Term Summary . . . . . . . . . . . . . . . . . . 49

4.1 Lunar Mission Cases . . . . . . . . . . . . . . . . . . . 59

4.2 Mission Cases Performance Comparison . . . . . . . . . . . . . 84

5.1 State Estimate Error Covariance Levels . . . . . . . . . . . . . 116

5.2 Fusion Simulation Test Cases . . . . . . . . . . . . . . . . 117

5.3 Scalar Update Algorithm . . . . . . . . . . . . . . . . . . . 123

5.4 Adaptive Measurement Noise Algorithm . . . . . . . . . . . . 126

5.5 Robustness Enhancement Scheme Performance Comparison . . 141 


\section{List of Figures}

1.1 Navigation Analysis Flowchart . . . . . . . . . . . . . 8

2.1 Geometry of the MCI frame and the LVLH frame. . . . . . . . 15

2.2 Cislunar transfer trajectory and libration point geometry. . . . 19

2.3 Exaggerated lunar formation orbit planes. . . . . . . . . . . . 21

2.4 Formation in the MCI frame centered at the reference point. . 24

2.5 Formation in the LVLH frame centered at the reference point. 25

2.6 Equilateral libration point tracking station visibility. . . . . . . 29

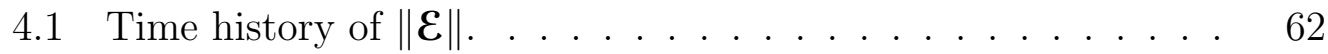

4.2 Numerical detection for Case 0. . . . . . . . . . . . . . . 64

4.3 Geometrical detection for Case 0 . . . . . . . . . . . . . . 65

4.4 Time history of the inertial position errors for Case 0 . . . . . 66

4.5 Time history of the inertial velocity errors for Case 0. . . . . . 67

4.6 Time history of the range residuals for Case 0 . . . . . . . . . 68

4.7 Numerical detection for Case 1. . . . . . . . . . . . . . . . . . 73

4.8 Geometrical arrangement for Case 1. . . . . . . . . . . . . . . 74

4.9 Geometrical detection for Case 1. . . . . . . . . . . . . . . . . 75

4.10 Time history of the inertial position errors for Case 1.0 . . . . . 77

4.11 Time history of the inertial velocity errors for Case 1.0. . . . . 78

4.12 Numerical detection for Case 2. . . . . . . . . . . . . . . . . . 81

4.13 Time history of the inertial position errors for Case 2.1. . . . . 82

4.14 Time history of the inertial velocity errors for Case 2.1. . . . . 83

4.15 Ideal three spacecraft formation in two dimensions. . . . . . . 88

4.16 Evaluation of $J$ for three spacecraft in two dimensions. . . . . 89

4.17 Proposed four spacecraft formation in two dimensions. . . . . 90

4.18 Proposed four spacecraft formation in three dimensions. . . . . 91

4.19 Evaluation of $J$ for four spacecraft restricted to a hemisphere. $\quad 92$ 
4.20 Evaluation of $J$ for four spacecraft restricted to a hemisphere. $\quad 93$

4.21 Time history of the position $\sigma_{\rho}^{2} \boldsymbol{\Gamma}^{-1}$ "upper bound" for Case 2.1. 97

4.22 Time history of the position $\mathfrak{J}^{-1}$ lower bound for Case 2.1. . . 102

4.23 Time history of the velocity $\mathcal{J}^{-1}$ lower bound for Case 2.1. . . 103

4.24 Time history of the position $\boldsymbol{J}_{\infty}^{-1}$ "upper bound" for Case 2.1. 105

4.25 Time history of the velocity $\mathcal{J}_{\infty}^{-1}$ "upper bound" for Case 2.1. . 106

4.26 Time history of the position $\sigma_{\rho}^{2} \mathcal{M}^{-1}$ forecast for Case 2.1. . . . 107

4.27 Time history of the velocity $\sigma_{\rho}^{2} \mathcal{M}^{-1}$ forecast for Case 2.1. . . . 108

4.28 Time history of the position $\sigma_{\rho}^{2}\left\|\mathcal{D}_{\mathbf{r}}\right\| \mathbf{I}$ forecast for Case 2.1. . . 111

4.29 Time history of the velocity $\sigma_{\rho}^{2}\left\|\mathcal{D}_{\mathbf{v}}\right\| \mathbf{I}$ forecast for Case 2.1. . . 112

5.1 Time history of the position errors for Case 3.1, Scheme 0. . . 118

5.2 Time history of the velocity errors for Case 3.1, Scheme 0. . . 119

5.3 Evaluation of second-order term $b_{f_{i}}$ for $\left\|\mathbf{r}_{j}\right\|=R_{\zeta}$. . . . . . 133

5.4 Evaluation of second-order terms $b_{h_{k}}$ and $B_{h_{k k}}$ for $\rho_{i j}=500 \mathrm{~km} .134$

5.5 Evaluation of second-order terms $b_{h_{k}}$ and $B_{h_{k k}}$ for $\rho_{i j}=10 \mathrm{~km} . \quad 135$

5.6 Navigation performance for various instances of Case 1.1. . . . 140 


\section{Chapter 1}

\section{Introduction}

As space exploration and space technologies advance, so do the ambition and complexity of space missions. In particular, the interest in formation flying missions is on the rise. Using innovative formations of multiple spacecraft, these missions can accomplish what would be extremely difficult and often too costly using only one spacecraft. With missions ranging from small formations for remote sensing and mapping to elaborate formations acting as interferometers and telescopes, precise and accurate inertial navigation will be vital for success. Although the increase in spacecraft creates a more complex mission, it also provides a measurement rich environment. For example, employing relative range measurements (between each spacecraft), a formation of $p$ spacecraft can provide $p(p-1) / 2$ unique relative measurements. Moreover, the use of these relative range measurements can directly contribute to improved navigation accuracy of the inertial state estimate. This increase in navigation accuracy comes at the cost of a decrease in robustness. Robustness, in this context, is defined to be the ability of the navigation filter to converge for a wide regime of state error covariances. This research provides a framework to analyze navigation performance for formation flying missions, and also presents methods to enhance robustness. 


\subsection{Review of Existing Literature}

The goal of navigation system design for formation flying missions is to maximize the navigation performance for the given mission under realistic conditions in the presence of uncertainties. This translates into a need for accurate design tools to aid in the development of formation flying mission architectures. For example, suppose there is flexibility in the spacecraft formation geometry or the location of the observers (Earth-based antennas, orbiting platforms, lunar beacons, etc.). It would be advantageous to have a straightforward methodology to compare different proposed navigation architectures based on the expected or desired resulting navigation performance. There is a similar need for robustness. For formation flying missions, this means accounting for the decrease in robustness experienced when fusing inertial and relative range measurements.

The notion of relating observability to the expected estimation error covariance is not new. For example, Ham [23] showed that the eigenvalues and eigenvectors of the state error covariance matrix can be used to provide insight into the observability of various linear combinations of the states. The problem considered here differs significantly from what is found in Ham, thus the solution approach deviates from what is found in the literature to date.

Observability of linear systems has been well studied. The condition for determining observability of linear systems is elegant and straightforward. Kalman [30-32] showed that the problem of determining observability reduces to a problem of linear algebra. Unfortunately, most real-world problems are 
modeled most accurately by nonlinear systems, and linearization methods (based on Taylor series expansions) are not satisfactory. Although conditions for nonlinear observability have also been well studied [22, 24, 29, 34], practically speaking implementing the resulting observability algorithms is typically extremely cumbersome and often ad hoc. It is quite common to examine a simplified mathematical model that has been linearized or made pseudo-linear.

Perhaps the two most popular examples found in literature connecting observability and navigation accuracy are the bearings-only targeting (BOT) problem $[10,42]$ and the target tracking problem using range-only measurements (ROM) $[48,49]$. In order to make the BOT and ROM problems manageable, the tracking scenarios are typically confined to two dimensions with the additional assumptions that the target is fixed or that the motion is such that it can be modeled as a piecewise constant linear system. While assumptions such as these can be justified for land and sea applications, they are not applicable to practical orbital applications. Consequently, it is not surprising that far less work has been done regarding the observability of spacecraft in orbital applications.

In the available literature, the work reported by Schneider [48] is nearest to the concept of observability considered here. The major differences being the two-dimensional assumption and that Schneider considers only relative navigation. Most other studies consider observability more as performance index to a control problem than as a measure representative of the expected navigation performance $[10,42,49]$. Generally the Fisher information matrix 
(which is related to observability) is used to determine the optimal observer maneuvers in terms of the reconstructability of the target state. Using the Fisher information matrix as a performance index is also common in missile tracking [37] and missile guidance [26] problems. Since each problem has it own set of unique assumptions, the corresponding Fisher information matrix, as well the resulting observability analysis, is specific to the given problem.

Observability can be used as a bound on the expected navigation accuracy. One of the most common examples of bounding the state error covariance is the Cramér-Rao lower bound [51]. Since this bound is based on the Fisher information matrix, literature on this topic is typically problem specific. Other notable examples of state error covariance bounds for Kalman filtering have been noted in regards to relating observability and controllability [16], augmented state applications [15], and multi-target multi-sensor tracking systems $[5,41]$. In the latter example, Bishop and Nabba [5] examine the use of the least-squares covariance to serve as a reference for the extended Kalman filter performance, and to study optimal sensor placement for a defense corridor guarding against approaching aircraft. Sands et al. [46] considers the use of a generalized version of the geometric dilution of precision metric commonly used in global positioning system receivers $[43,56]$ to provide a rough estimate of the solution accuracy.

To maximize the benefits of the measurement rich environment associated with formation flying missions, it is desirable to fuse relative range measurements whenever possible. It is known that fusing relative range mea- 
surements with inertial range measurements can lead to navigation filter divergence $[27,28]$. It would be helpful in the design process to understand the circumstances, as well as the critical operating regimes, that causes filter divergence when fusing relative range measurements.

Examination of the convergence properties of the Kalman filter is not new. Engineers have been studying the causes of Kalman filter divergence since the early 1960s. Filter divergence occurs when the errors of the filter state estimate are significantly larger than the corresponding estimation error covariances predicted by filter theory. Early studies of orbital applications report nonlinearities as a major contributor to filter divergence in the linearized and extended versions of the Kalman filter $[17,47,52]$. The effects of nonlinearities arises in a variety of ways. Large errors in the initial state estimate or inaccuracy of the state error covariance can increase nonlinearities, which may lead to divergence [52]. Evaluating the linearization about a more accurate state estimate reduces the effect of nonlinearities [47]. Another interesting finding is the somewhat paradoxical issue of measurement accuracy. Ironically, the smaller the measurement noise (that is, the more accurate the sensor), the more significant the neglected nonlinearities become, which in turn results in a greater risk of filter performance degradation and divergence $[17,47,52]$.

Denham and Pines [17] state that when the measurement nonlinearity is comparable to the measurement error, the calculated state error covariance becomes inaccurate, leading to "nonoptimal" filter performance. They illustrate this by examining the iterative use of a linear estimator scheme and 
second-order techniques to reduce the nonlinearities yielding a more accurate state estimate error covariance. While the methods of Denham and Pines are only valid for a restricted class of problems, one interesting finding was that the nonlinearities acted as a nonzero-mean bias which must be accounted for to accurately compute the state estimate error covariance. Updated algorithms accounting for the nonzero-mean bias could require the inclusion of third and fourth order moments of the state estimate error. Coincidentally, advances in nonlinear filter theory soon thereafter led to the Gaussian second-order filter (discussed later in this dissertation) that considers third and fourth order moments and incorporates the appropriate residual bias compensation and residual covariance increase.

The work on filter divergence served as the basis for future studies on filter performance for rendezvous and radar tracking. In particular, Widnall [55] used Gaussian second-order filter terms to enlarge the region of convergence when employing range measurements during rendezvous. His rendezvous simulation examines only the second-order terms associated with a scalar range measurement. Widnall demonstrated that while the linearized and extended Kalman filters perform satisfactorily for an initial set of range measurements, the processing of a second set results in filter divergence due to a large discrepancy between the actual state errors and the computed state error covariance. While Widnall notes that this is caused by the filter having a false sense of outof-plane observability, in light of the results presented later in this dissertation it should also be noted here that due to the rendezvous maneuvers the second 
set of range measurements is an order of magnitude smaller than the first. Next, by including the second-order terms to estimate the bias and covariance increase due to nonlinearities, the estimation algorithm successfully processed the second set of range measurements, thereby demonstrating an increase in the region of convergence. Other studies examined the use of artificial measurement noise to enlarge the covariance region by avoiding the measurement accuracy paradox $[17,52]$. The Gaussian second-order filter and artificial measurement noise are among the techniques considered in this dissertation for application to formation flying.

More recently, radar tracking algorithms have been reported that employ statistical methods to estimate the bias and covariance due to using converted measurements $[36,44]$. The so-called converted measurement algorithms attempt to minimize measurement nonlinearities by "converting" the nonlinear polar radar measurements of range, azimuth, and elevation angle into a pseudo-linear cartesian measurement of the components of the position. While this method has demonstrated better performance than the standard extended Kalman filter, the additional computation of a bias and covariance resulting from the original measurement nonlinearity is required [36]. Examining the converted measurement Kalman filter, Park and Lee [44] derived a "mixed coordinate" extended Kalman filter (no measurement conversion) that implements a scalar update scheme and uses an estimated azimuth covariance to compute the range measurement noise covariance in an adaptive manner. The scalar update scheme and adaptive measurement noise are also techniques 
considered in this dissertation.

\subsection{Research Contributions}

One contribution of this research is the navigation observability analysis methodology, which provides a means of forecasting the approximate state estimate accuracy based on the formation-observer geometry (time history). This is accomplished using measures of system observability to relate the formationobserver geometry to the resulting state error covariance, as described in Figure 1.1. The proposed techniques can also be used in design of the navigation infrastructure. For example, the observability measure can be used during the design process to determine which observer geometries have the capacity to increase observability and which observer geometries should be avoided.

Figure 1.1: Navigation Analysis Flowchart

\begin{tabular}{|c|c|c|c|c|}
\hline Observer & $\S 4.2$ & Observability & $\S 4.3$ & Covariance \\
\hline Geometry & $\Longleftrightarrow$ & Measure & $\Longrightarrow$ & Forecast \\
\hline
\end{tabular}

Examination of the validity of several different measures of observability within in the context of orbital applications is the first step in the process. In particular, $(i)$ linear time-invariant, $($ ii $)$ nonlinear time-invariant, and (iii) linear time-varying observability measures are compared. A relationship between the linear and nonlinear time-invariant observability measures is derived. 
The linear time-invariant measure is then used to demonstrate how the spacecraft formation-observer geometry can effect observability and correspondingly effect the navigation performance. The linear observability measures are directly related to the state error covariance to offer bounds (or rough forecasts) of the expected solution accuracy. In the time-varying case, an explicit relationship between the observability Gramian and the Fisher information matrix is derived such that the state error covariance can then be related to the observability using the Cramér-Rao lower bound. This last result is compared with the existing generalized geometric dilution of precision metric.

The issue of fusing relative and inertial range measurements in the formation flying scenario is solved here yielding a practical algorithm consistent with the second-order filter studied in the 1960s. An explanation of why previous ad hoc methods worked is presented. In particular, this work thoroughly examines the significance of the quadratic nonlinearities in the measurement update, as well as in the propagation of the dynamics. Moreover, analytical bounds are constructed that explicitly link the state estimate error covariance to the second-order terms, which in turn also provides a method to determine exactly when the quadratic nonlinearities become significant. These results definitively explain what causes nonlinearities in relative range measurements to become more significant than in inertial range measurements. Finally, all of this is accomplished in the natural context of an inertial frame and without the artificial conversion of the range measurements or the need for azimuth to compute the range measurement noise covariance. 


\subsection{Organization of the Dissertation}

The organization of this dissertation is as follows: The necessary mathematical models such as coordinate systems are discussed in Chapter 2. Also, the benchmark formation flying scenarios employed in the analysis are presented. The filter algorithms are presented in Chapter 3. The extended Kalman filter serving as the basis for much of the navigation analysis is described. The modified Gaussian second-order filter, which is an essential part of the measurement fusion analysis, is also described. Chapter 4 presents the analysis of the impact of system observability on overall navigation performance. In particular, several key observability metrics are examined assessing the effect of observer and formation geometries, as well as the corresponding solution accuracy that can be expected. Chapter 5 considers the complications and overall decrease in robustness due to fusing inertial and relative range measurements. Among these considerations are several first-order fixes and a thorough examination of the second-order terms, which results in a practical design rule-of-thumb regarding robustness. Finally, Chapter 6 summarizes the major results of the research and offers direction for future research. 


\section{Chapter 2}

\section{Foundation of Benchmark Formation}

This chapter describes the notation, coordinate systems, and benchmark problem used throughout this dissertation.

\subsection{Mathematical Notation and Parameters}

The following notation convention is adopted: lowercase bold letters (or symbols) denote vectors, uppercase bold letters (or symbols) denote matrices, lowercase italicized letters (or symbols) denote scalars, and uppercase italicized letters typically denote either a scalar component of a matrix, an object label, or an operator. For example, the operation $E[x]$ denotes the expected value of the random variable $x$, which is also known as the mean or average value

of $x$, and can be used to define the variance of $x$ as $\sigma^{2}=E\left[x^{2}\right]-E[x]^{2}$. The vector or matrix 2-norm is denoted by $\|\cdot\|$. The $i^{\text {th }}$ component of the set of eigenvalues $\boldsymbol{\lambda}(\cdot)$ is denoted by $\lambda_{i}(\cdot)$.

The symbol $\mathbb{R}$ represents the field of all real numbers. The function $\delta(t-\tau)$ represents the Dirac delta function (zero for all $t \neq \tau$ ) and is defined by the integral

$$
\int_{-\infty}^{\infty} f(t) \delta(t-\tau) d t=f(\tau)
$$


for finite-valued functions $f(t)$ that are continuous at $t=\tau$. The Kronecker delta $\delta_{i j}$ is defined by

$$
\delta_{i j}:= \begin{cases}1 & i=j \\ 0 & i \neq j\end{cases}
$$

In general, an overbar $\left(^{-}\right)$denotes a mean or average value (e.g. $\bar{x}=$ $\left.\left(x_{1}+\cdots+x_{n}\right) / n\right)$, a hat accent $\left(^{\wedge}\right)$ denotes an estimated quantity (e.g. $\hat{x}$ is the estimated value of $x$ ), a dot accent $\left({ }^{*}\right)$ indicates a temporal derivative (e.g. $\dot{x}=d x / d t$ ), and a prime accent (') indicates a spatial derivative (e.g. $f^{\prime}=\partial f(\partial x)$. The superscripts " $T$ " and " -1 " indicate the transpose and inverse, respectively; while the superscripts "-" and "+" indicate an a priori value and an a posteriori value (i.e. values immediately before and after a measurement update), respectively.

The subscript "*" indicates a value associated with the spacecraft formation reference point, while the subscripts " $\oplus$ " and " " denote values of the celestial bodies the Earth and the Moon, respectively. In particular, Table 2.1 lists key Earth and lunar constants used in this study, where $\mu$ is the gravitational constant of the body, $R_{\oplus}$ and $R_{\hookrightarrow}$ are the mean equatorial radii of the bodies, $a_{\oplus / \mathcal{\Omega}}$ is the mean distance between the Earth and the Moon, and $\omega_{\oplus / \mathcal{B}} \approx 13.2^{\circ}$ day is the mean motion of the Earth-Moon system. It is assumed that the Earth and Moon are uniform mass, spherical bodies of

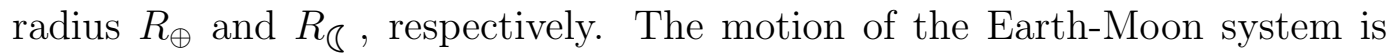
assumed circular such the bodies remain a constant distance $a_{\oplus / \varsigma}$ apart while rotating about their common center of mass at a rate of $\omega_{\oplus / \varsigma}$. (Although in 
subsequent sections $R$ is reserved to represent a component of the measurement noise covariance, the radius of a body is easily distinguished by noting the celestial subscript.)

Table 2.1: Celestial Body Parameters [6]

\begin{tabular}{|c|c|c|c|c|c|}
\hline$\mu_{\oplus}$ & $=$ & $398,600.4 \mathrm{~km}^{3} / \mathrm{s}^{2}$ & $\mu_{\zeta}$ & $=$ & $4,902.8 \mathrm{~km}^{3} / \mathrm{s}^{2}$ \\
\hline$R_{\oplus}$ & $=$ & $6,378.14 \mathrm{~km}$ & $R_{\overparen{乃}}$ & $=$ & $1,737.4 \mathrm{~km}$ \\
\hline$a_{\oplus / \varsigma}$ & $=$ & $384,400 \mathrm{~km}$ & $\omega_{\oplus / ठ}$ & $=$ & 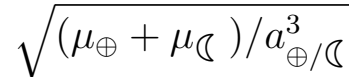 \\
\hline
\end{tabular}

\subsection{Coordinate Systems and Frame Transformations}

Three basic coordinate systems are used. The inertial (celestial) bodycentered equatorial system has its origin at the body center of mass and its fundamental plane $\left(X_{I^{-}} Y_{I}\right)$ is aligned with the orientation of the equatorial plane of the central body at the J2000 epoch (January 1, 2000, 12 noon Terres-

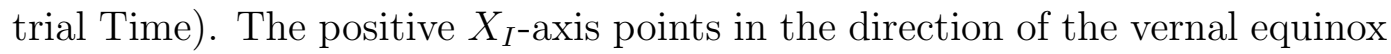

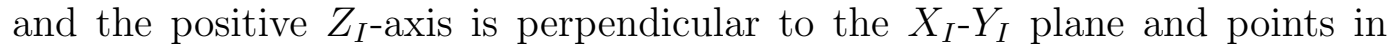
the direction of the body north pole vector, thus completing the right-handed orthogonal basis for the system. Depending upon the central body, two variations of the body-centered inertial frame are employed: the geocentric inertial frame (not to be confused with the standard Earth-centered inertial frame) and the Moon-centered inertial (MCI) frame. While the geocentric inertial 
frame is centered at the Earth, the axes of this frame are aligned with those of the MCI frame.

These frames are referred to as "inertial" because they are fixed with respect to the stars (except for the precision of the equinoxes) and do not rotate with the central body. However, this terminology only applies in the absence of perturbing bodies. If perturbing bodies were included in the dynamics, although the frames would still be body-centered and fixed (non-rotating), they would no longer be inertial. Since, as discussed in Section 2.3.2, the dynamics considered in this dissertation do not included perturbing bodies, these frames are appropriately considered inertial. Figure 2.1 illustrates the body-centered inertial coordinate system.

The local-vertical local-horizontal (LVLH) frame is used to describe the spacecraft formation. The LVLH frame has its origin at an orbiting reference point and its fundamental plane $\left(X_{L^{-}} Z_{L}\right)$ aligned with the orbit plane of the reference point. The positive $Z_{L}$-axis points in the direction opposite the reference point radius vector and the positive $Y_{L}$-axis is perpendicular to the orbital plane and points in the direction opposite the orbital angular momentum vector, thus completing the righthanded orthogonal basis for the system. The LVLH frame is also illustrated in Figure 2.1.

The transformation matrix between the inertial and local coordinate systems can be obtained using the directional unit vectors of the LVLH frame expressed in the body-centered inertial frame. The resulting transformation matrix $\mathbf{T}$, with functional dependencies on time suppressed for compactness, 


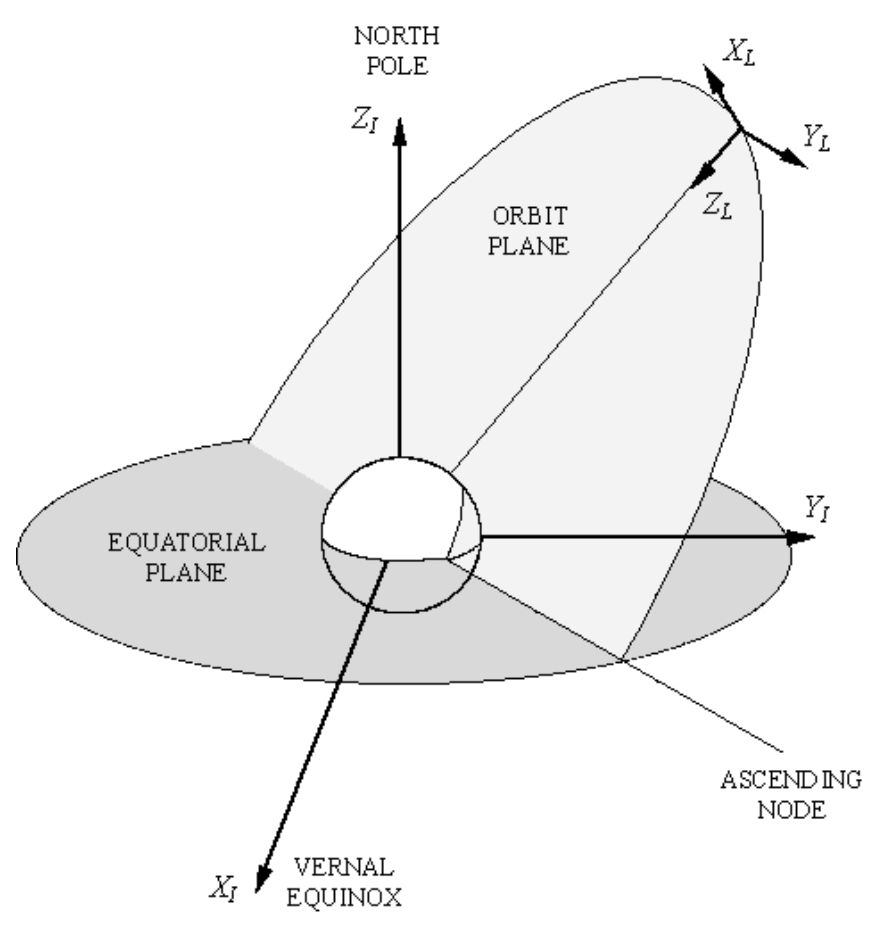

Figure 2.1: Geometry of the MCI coordinate system and the LVLH coordinate system. Image adapted courtesy of NASA [2].

is expressed as

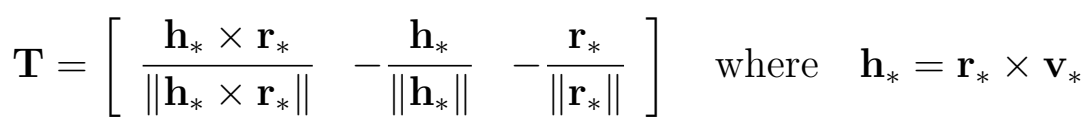

where the subscript " $*$ " indicates values for the reference point, and the vectors $\mathbf{r}, \mathbf{v}$, and $\mathbf{h}$ are body-centered inertial representations of the position, velocity, and specific angular momentum vectors, respectively. (Although outside of this section $\mathbf{h}$ represents the measurement model, the specific angular momentum of the reference point is easily distinguished by noting the "*" subscript.)

If the reference orbit is circular, $\mathbf{v}_{*}$ is perpendicular to $\mathbf{r}_{*}$, consequently 
$\mathbf{V}_{*}$ is parallel to $\mathbf{h}_{*} \times \mathbf{r}_{*}$ and can be used in its place in Eq. (2.3). Additionally, for a circular reference orbit the following properties hold for all time:

$$
\begin{array}{ll}
\left\|\mathbf{r}_{*}\right\|=a_{*} & \left\|\mathbf{v}_{*}\right\|=\sqrt{\mu / a_{*}} \\
\left\|\mathbf{h}_{*}\right\|=\left\|\mathbf{r}_{*}\right\|\left\|\mathbf{v}_{*}\right\|=\sqrt{\mu a_{*}} & \left\|\boldsymbol{\omega}_{*}\right\|=\omega_{*}=\left\|\mathbf{v}_{*}\right\| /\left\|\mathbf{r}_{*}\right\|=\sqrt{\mu / a_{*}^{3}}
\end{array}
$$

where $\mu$ is the gravitational constant of the central body, $a_{*}$ is the semi-major axis of the reference point orbit, and $\boldsymbol{\omega}_{*}$ is the angular velocity vector of the reference point orbit. Furthermore, when no perturbations are present the angular velocity vector is constant and parallel to the specific angular momentum vector such that $\boldsymbol{\omega}_{*}=\left[\begin{array}{lll}\omega_{1} & \omega_{2} & \omega_{3}\end{array}\right]^{T}=\mathbf{h}_{*} / a_{*}^{2}$.

With the angular velocity vector known, the time-derivative of the transformation matrix, $\mathbf{\mathbf { T }}$, can then be computed using Eq. (2.5), yielding

$$
\dot{\mathbf{T}}=\left[\boldsymbol{\omega}_{*} \times\right] \mathbf{T} \quad \text { where } \quad\left[\boldsymbol{\omega}_{*} \times\right]:=\left[\begin{array}{ccc}
0 & -\omega_{3} & \omega_{2} \\
\omega_{3} & 0 & -\omega_{1} \\
-\omega_{2} & \omega_{1} & 0
\end{array}\right]
$$

Once $\mathbf{T}$ and $\dot{\mathbf{T}}$ are known, any local (LVLH) position vector $\mathbf{r}^{L}$ and corresponding local velocity vector $\mathbf{v}^{L}$ can be expressed in the body-centered inertial frame using the following transformations:

$$
\begin{aligned}
& \mathbf{r}(t)=\mathbf{r}_{*}(t)+\mathbf{T}(t) \mathbf{r}^{L}(t) \\
& \mathbf{v}(t)=\mathbf{v}_{*}(t)+\mathbf{T}(t) \mathbf{v}^{L}(t)+\dot{\mathbf{T}}(t) \mathbf{r}^{L}(t)
\end{aligned}
$$

The third coordinate system employed is the body-centered rotating frame and is used to describe the equilibrium positions resulting from the circular restricted three-body problem dynamics of the Earth-Moon system. The rotating frame is aligned with the body-centered inertial frame (at time $t_{0}$ ) 
and the axes of its fundamental plane $\left(X_{R^{-}} Y_{R}\right)$ rotate at a constant rate $\dot{\theta}$ about

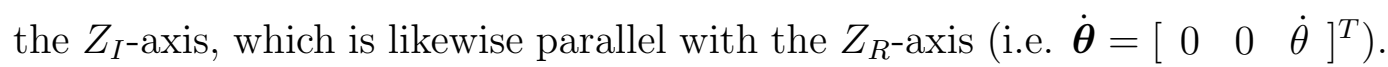

The transformation matrix from the body-centered rotating frame to the body-centered inertial frame is given by

$$
\mathbf{T}_{Z_{I}}(t)=\left[\begin{array}{ccc}
\cos \theta & -\sin \theta & 0 \\
\sin \theta & \cos \theta & 0 \\
0 & 0 & 1
\end{array}\right]
$$

where $\theta=\theta(t)$ is the angle from the $X_{I}$-axis to the $X_{R}$-axis measured positive counterclockwise about the $Z_{I^{-}}$axis. The time-derivative of the transformation matrix in Eq. 2.7 is

$$
\dot{\mathbf{T}}_{Z_{I}}(t)=[\dot{\boldsymbol{\theta}} \times] \mathbf{T}_{Z_{I}}(t)=\dot{\theta}\left[\begin{array}{ccc}
-\sin \theta & -\cos \theta & 0 \\
\cos \theta & -\sin \theta & 0 \\
0 & 0 & 0
\end{array}\right]
$$

Rotating frame position vectors, $\mathbf{r}^{R}$, and corresponding rotating frame velocity vectors, $\mathbf{v}^{R}$, can be expressed in the body-centered inertial frame as follows:

$$
\begin{aligned}
& \mathbf{r}(t)=\mathbf{T}_{Z_{I}}(t) \mathbf{r}^{R}(t) \\
& \mathbf{v}(t)=\mathbf{T}_{Z_{I}}(t) \mathbf{v}^{R}(t)+\dot{\mathbf{T}}_{Z_{I}}(t) \mathbf{r}^{R}(t)
\end{aligned}
$$

\subsection{Lunar Benchmark Problem Formulation}

A two-part lunar mission was chosen to illustrate the concepts introduced in Chapter 1. This section describes each segment of the lunar mission and develops the models that will be used in the navigation analysis. 


\subsubsection{Mission and Formation Description}

The lunar mission under consideration consists of two flight segments: a cislunar (i.e. Earth-Moon) transfer trajectory and a circular polar orbit. To simplify the geometry it has been assumed in both segments that the equatorial plane of the Moon is coplanar with the Earth-Moon orbit plane. This is not unreasonable considering that the inclination between these planes is less than $7^{\circ}[3,6]$.

During the cislunar transfer trajectory a single cluster of spacecraft is examined in two dimensions in the geocentric inertial frame. However, while this frame has been centered at the Earth to make visualization more intuitive, the axes of this frame are actually aligned with the MCI frame. This allows for two-dimensional analysis using the MCI $X_{I^{-}} Y_{I}$ plane by assuming the transfer trajectory is coplanar with the Earth-Moon orbit plane. Figure 2.2 shows the cislunar transfer trajectory (black), the trajectory of the Moon (red), and the Earth (cyan) at the origin. The dotted lines denote parts of the complete trajectories not traversed during the transfer. The triangles and diamond denote the "snapshot" locations of the five Earth-Moon libration points when the Moon crosses the $X_{I^{-}}$axis at time $t_{\text {trans }}$.

The significance of the libration points will be addressed in more detail after describing the circular polar orbit segment. For now consider the placement of tracking stations at $L_{4}$ and $L_{5}$ during the cislunar transfer segment. These tracking stations provide range measurements that have cyclical trends

with distances (from the stations to the cluster) ranging from a minimum of 


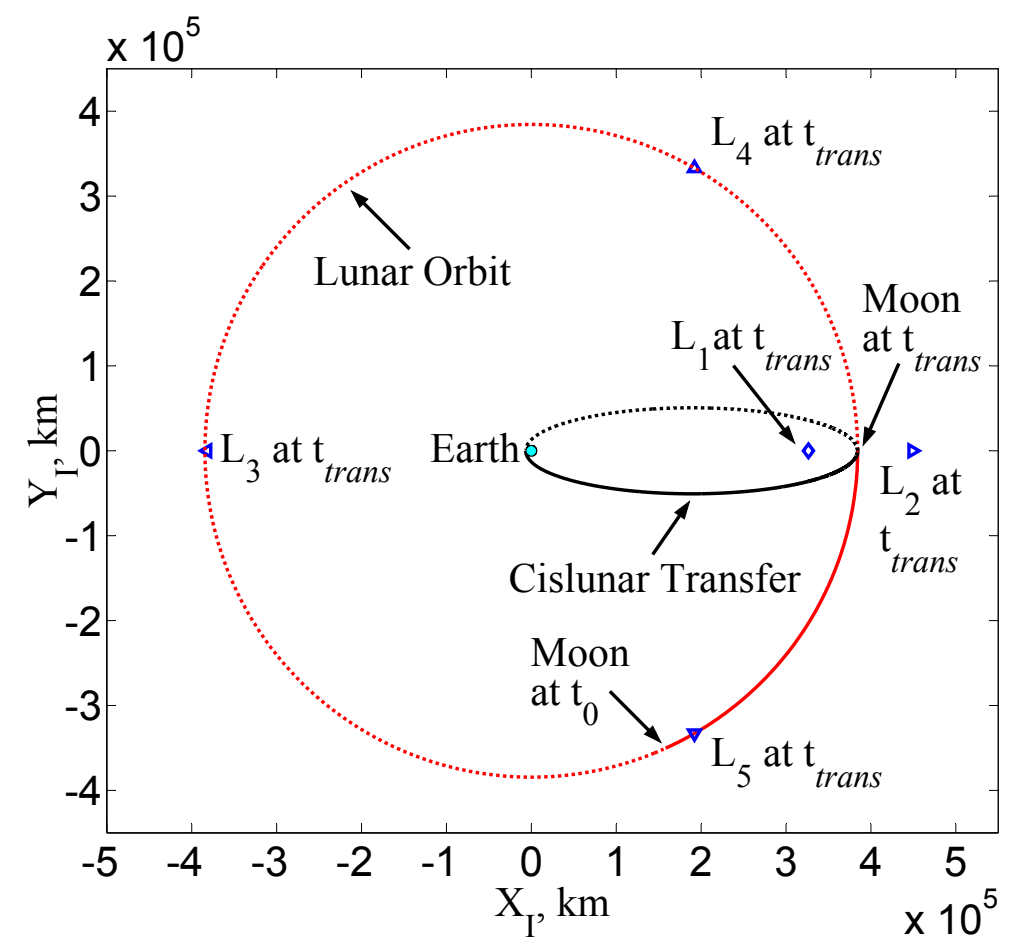

Figure 2.2: Cislunar transfer trajectory and libration point geometry (at time $\left.t_{\text {trans }}\right)$.

about $1.89 \times 10^{5} \mathrm{~km}$ to a maximum of about $4.85 \times 10^{5} \mathrm{~km}$.

The transfer is accomplished by ignoring the gravitational effect of the Moon and implementing a Hohmann transfer, which is the minimum $\Delta v$ transfer possible [3]. Applying the principles of a Hohmann transfer to the cislunar transfer results in the following initial conditions,

$$
\mathbf{r}\left(t_{0}\right)=\left[\begin{array}{c}
-r_{\text {peri }} \\
0
\end{array}\right] \quad, \quad \mathbf{v}\left(t_{0}\right)=\left[\begin{array}{c}
0 \\
-v_{\text {peri }}
\end{array}\right]
$$

where $r_{\text {peri }}$ and $v_{\text {peri }}$ are the perigee radius and perigee velocity of the transfer 
orbit. Choosing a value of $r_{\text {peri }}=R_{\oplus}+300 \mathrm{~km}$, the semi-major axis $a_{*}$ and perigee velocity $v_{\text {peri }}$ of the transfer orbit can be computed via,

$$
a_{*}=\frac{a_{\oplus / \varsigma}+r_{\text {peri }}}{2}, \quad v_{\text {peri }}=\sqrt{\mu_{\oplus}\left(\frac{2}{r_{\text {peri }}}-\frac{1}{a_{*}}\right)}
$$

which results in a transfer time of $t_{\text {trans }}=\pi \sqrt{a_{*}^{3} / \mu_{\oplus}} \approx 5$ days. Although ignoring lunar gravity oversimplifies the dynamics during the lunar approach, it provides a sufficient trajectory to analyze the observer location geometry, the main focus of this mission segment. This simplification also allows for the use of a consistent dynamical model equation throughout all mission segments, since only two-body dynamics are used in the subsequent mission cases.

Upon reaching the Moon the cluster separates and multiple spacecraft in formation about a reference point in a $100 \mathrm{~km}$ altitude circular polar orbit are examined in the MCI frame. As illustrated in Figure 2.3, the polar orbit is oriented such that the line of nodes is parallel to the $Y_{I^{-}}$axis, which results in the orbit plane being coplanar with the $Y_{I^{-}} Z_{I}$ plane. As shown in Figure 2.2, at the start of this mission segment the orientation of the Earth is such that the Earth-Moon line is initially aligned with the $X_{I}$-axis and then slowly rotates $\left(13.2^{\circ} /\right.$ day $)$ in the $X_{I^{-}} Y_{I}$ plane counterclockwise about the $Z_{I^{-}}$axis. Consequently, if it were desired to keep the orbit plane perpendicular to the Earth-Moon line scheduled maneuvers would be required (no lunar orbit exists such that the regression of nodes due to the second zonal harmonic, $J_{2}$, matches the angular rotation rate of the Earth-Moon system). The significance of the geometry between the node line and the Earth-Moon line is discussed more 


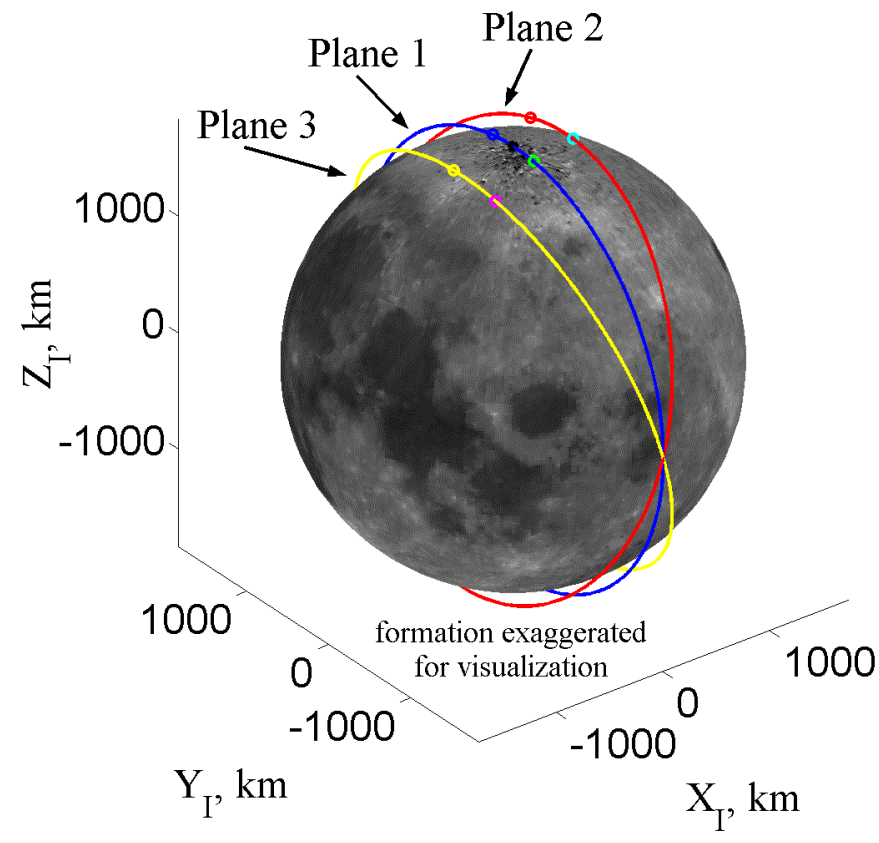

Figure 2.3: Exaggerated lunar formation orbit planes.

fully in Section 4.2.2. This mission configuration can be used for estimating the far side lunar gravity coefficients, lunar surface mapping, or as a far side communication relay.

The navigation and observability analysis of the circular polar orbit mission segment examines two formation variations: a one-plane, two-spacecraft formation and a three-plane, six-spacecraft formation. In both formations, each plane consists of two spacecraft travelling in a $100 \mathrm{~km}$ altitude circular

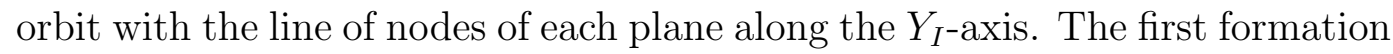


plane employs the reference point orbit. The second formation builds on the first by adding two secondary planes, each tilted a distance defined as $b=10$ $\mathrm{km}$ in opposite directions away from the first plane. Within each of the three planes, two spacecraft slots are staggered about the projected argument of latitude of the reference point such that the range between coplanar spacecraft is also chosen to be $b=10 \mathrm{~km}$. In terms of orbital elements the distance $b$ corresponds to an inclination difference between the secondary planes and the reference point plane $(\Delta \iota)$ and an angular spacing between coplanar spacecraft $(\Delta \nu)$ of $2 \sin ^{-1}\left(b /\left(2 a_{*}\right)\right) \approx 0.3^{\circ}$, where $a_{*}$ is the semi-major axis of the circular

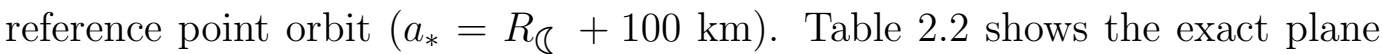
tilt angle $\left(\Delta \iota_{i}\right)$ and lead/lag slot angle $\left(\Delta \nu_{i}\right)$ for spacecraft $i$ relative to the reference point plane and the projected argument of the latitude, respectively. Figure 2.3 illustrates an exaggerated formation $(b=500 \mathrm{~km})$ for visualization purposes. The location of each spacecraft in the exaggerated formation at time $t_{0}$ is indicated by an "O" marker.

Table 2.2: Lunar Formation Parameters

\begin{tabular}{|c|c|c|c|c|c|c|c|}
\hline$\Delta \iota_{i}=\tau_{i} \Delta \iota$ & $\Delta \nu$ & \multicolumn{2}{|c|}{ Plane 1} & \multicolumn{2}{|c|}{ Plane 2} & \multicolumn{2}{|c|}{ Plane 3} \\
\hline Spacecraft Number: & $i=$ & 1 & 2 & 3 & 4 & 5 & 6 \\
\hline Plane Tilt Factor: & $\tau_{i}=$ & 0 & 0 & 1 & 1 & -1 & -1 \\
\hline $\begin{array}{l}\text { Slot Lead/Lag Factor: } \\
\text { Trajectory Color: }\end{array}$ & $\gamma_{i}=$ & $-1 / 2$ & $1 / 2$ & $-5 / 6$ & $1 / 6$ & $-1 / 6$ & $5 / 6$ \\
\hline
\end{tabular}

The reference point has a circular orbital velocity of $v_{*}=\sqrt{\mu_{\zeta} / a_{*}}$ and an orbital period of $t_{o p}=2 \pi \sqrt{a_{*}^{3} / \mu_{\S}} \approx 2$ hours. The initial position $\left(\mathbf{r}_{i}\right)$ and velocity $\left(\mathbf{v}_{i}\right)$ of the $i^{t h}$ spacecraft in the formation at time $t=t_{0}$ is obtained by 
rotating the initial position $\left(\mathbf{r}_{*}\right)$ and velocity $\left(\mathbf{v}_{*}\right)$ of the reference point such that,

$$
\mathbf{r}_{i}\left(t_{0}\right)=\mathbf{T}_{Y_{I}}\left(t_{0}\right) \mathbf{T}_{X_{I}}\left(t_{0}\right) \mathbf{r}_{*}\left(t_{0}\right) \quad, \quad \mathbf{v}_{i}\left(t_{0}\right)=\mathbf{T}_{Y_{I}}\left(t_{0}\right) \mathbf{T}_{X_{I}}\left(t_{0}\right) \mathbf{v}_{*}\left(t_{0}\right)
$$

where

$$
\mathbf{r}_{*}\left(t_{0}\right)=\left[\begin{array}{c}
0 \\
0 \\
a_{*}
\end{array}\right] \quad, \quad \mathbf{T}_{Y_{I}}\left(t_{0}\right)=\left[\begin{array}{ccc}
\cos \left(\Delta \iota_{i}\right) & 0 & \sin \left(\Delta \iota_{i}\right) \\
0 & 1 & 0 \\
-\sin \left(\Delta \iota_{i}\right) & 0 & \cos \left(\Delta \iota_{i}\right)
\end{array}\right]
$$

and

$$
\mathbf{v}_{*}\left(t_{0}\right)=\left[\begin{array}{c}
0 \\
-v_{*} \\
0
\end{array}\right] \quad, \quad \mathbf{T}_{X_{I}}\left(t_{0}\right)=\left[\begin{array}{ccc}
1 & 0 & 0 \\
0 & \cos \left(\Delta \nu_{i}\right) & -\sin \left(\Delta \nu_{i}\right) \\
0 & \sin \left(\Delta \nu_{i}\right) & \cos \left(\Delta \nu_{i}\right)
\end{array}\right]
$$

Using these initial conditions, trajectories for each spacecraft can be generated for $t \in\left[t_{0}, t_{f}\right]$. Figures 2.4 and 2.5 show the spacecraft trajectories about the reference point for $t_{f}=t_{o p} / 2$ in the MCI and LVLH frames, respectively. A final time of $t_{f}=t_{o p} / 2$ was used to allow for easier viewing of the individual spacecraft trajectories in Figure 2.4 by preventing overlapping trajectories. The trajectory color code for each spacecraft can be found in Table 2.2. The location of each spacecraft at time $t_{0}$ is indicated by an "o" marker.

A well known consequence of a system of two celestial bodies is the existence of libration (or Lagrange) points. Libration points are equilibrium points resulting from the natural dynamics of the system. The study of these dynamics is commonly known as the circular restricted three-body problem (CR3BP) [50], where the third body is of relatively negligible mass, such as 

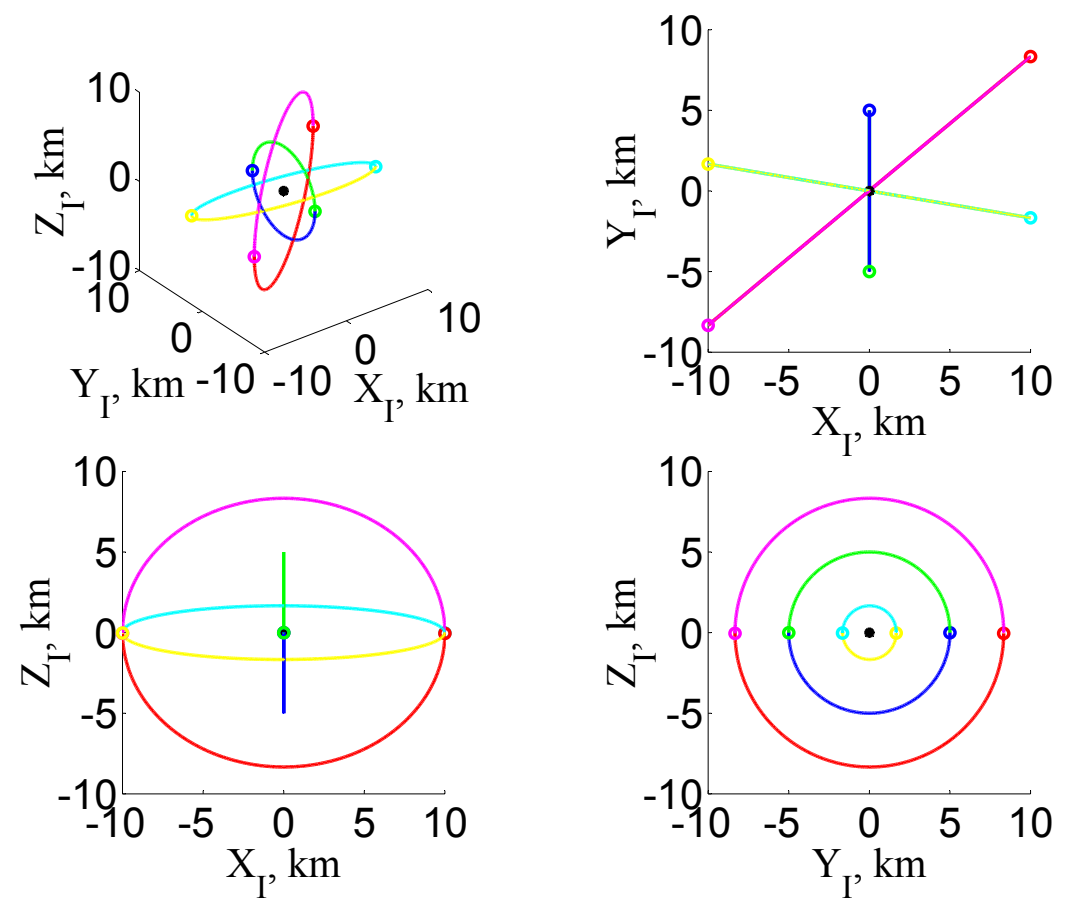

Figure 2.4: Formation in the MCI frame centered at the reference point.

a spacecraft. The relative locations of the libration points of the CR3BP are found by solving the equations of motion in the rotating frame for the locations where the velocity and acceleration are zero. The resulting locations for the libration points are fixed in the rotating frame. Since the libration points naturally maintain the same geometry with respect to the two celestial bodies, in this case the Earth and the Moon, they make ideal observer locations in navigation architectures for long-term Earth-Moon operations. Figure 2.2 shows the geometry of the five libration points $\left(L_{1}-L_{5}\right)$ in the geocentric inertial 

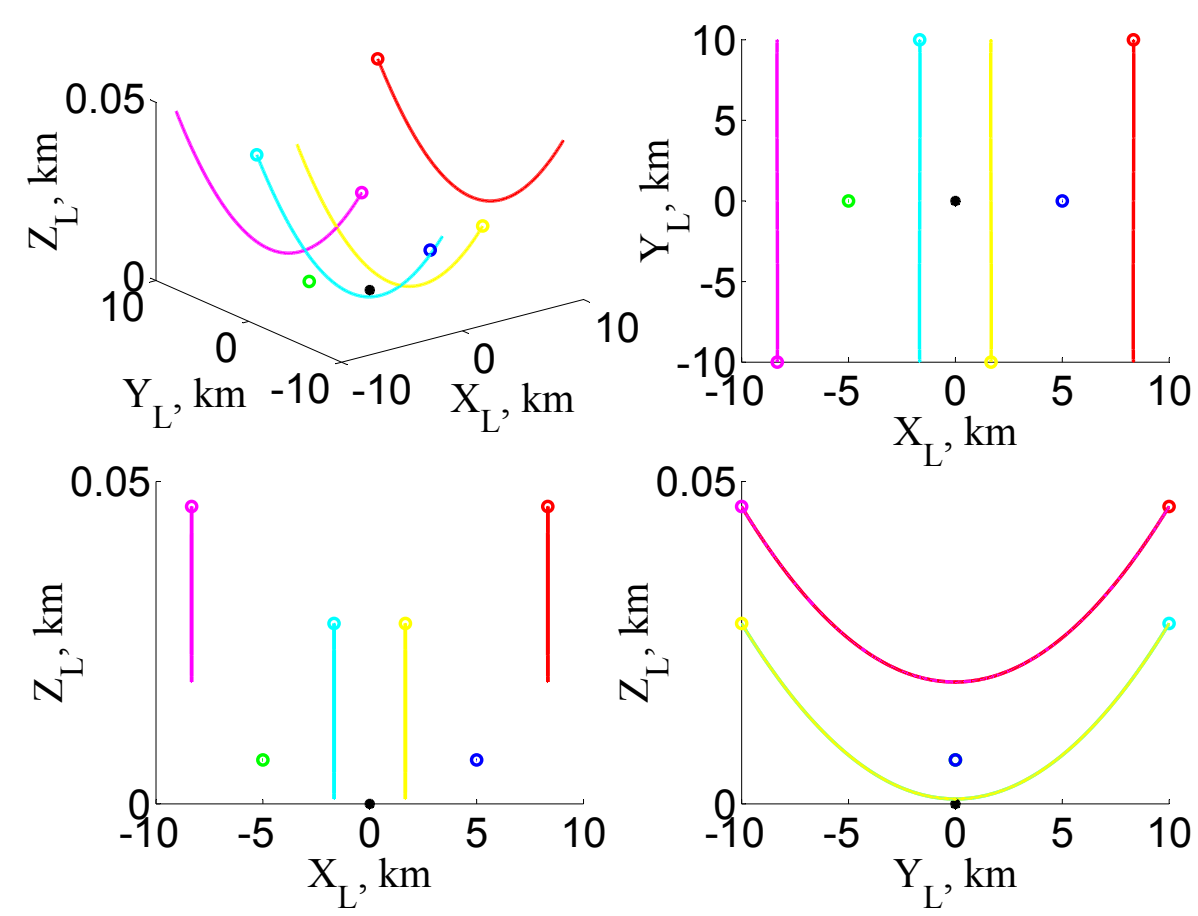

Figure 2.5: Formation in the LVLH frame centered at the reference point.

frame when the Earth-Moon line is aligned with the $X_{I \text {-axis. }}$

The exact location of the Earth-Moon libration points in the Mooncentered rotating frame, which has the $X_{R}$-axis aligned with the Earth-Moon line and $\dot{\theta}=\omega_{\oplus / \mathcal{C}}$, can be expressed for all time as seen in Table 2.3 (to four decimal places). Note that the $x$ components of the positions in Table 2.3 must be shifted by $a_{\oplus / ৫}$ when used for the cislunar transfer segment since this mission instead uses an Earth-centered frame. The location of the libration points at anytime $t$ is found in the body-centered inertial frame by rotating $\mathbf{r}_{L_{i}}^{R}$ 


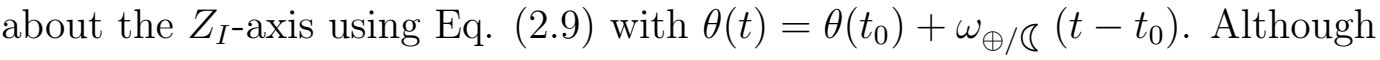
$\theta\left(t_{0}\right)$ is different for each mission segment, they are related since the cislunar transfer segment ends where the circular polar orbit segment begins. In particular, $\theta\left(t_{0}\right)$ for the first segment must be such that after a transfer time of $t_{\text {trans }}, \theta\left(t_{0}+t_{\text {trans }}\right)$ for the first segment is equal to $\theta\left(t_{0}\right)=0$ for the second segment. Consequently, $\theta\left(t_{0}\right)=-\omega_{\oplus / \diamond} t_{\text {trans }}$ for the first segment.

\section{Table 2.3: Initial Libration Point Locations}

\begin{tabular}{|c|c|c|c|c|c|}
\hline & $\mathbf{r}_{L_{i}}^{R}$ & $x_{L_{i}}^{R}$ & $z_{L_{i}}^{R}$ & \multicolumn{2}{|c|}{$T$} \\
\hline$i=$ & 1 & 2 & 3 & 4 & 5 \\
\hline$x_{L_{i}}^{R}=a_{\oplus / \Omega} \times$ & -0.1509 & +0.1678 & -1.9929 & -0.5000 & -0.5000 \\
\hline$y_{L_{i}}^{R}=a_{\oplus / \Omega} \times$ & 0 & 0 & 0 & +0.8660 & -0.8660 \\
\hline$z_{L_{i}}^{R}=a_{\oplus / \Omega} \times$ & 0 & 0 & 0 & 0 & 0 \\
\hline
\end{tabular}

In the context of the CR3BP (no additional perturbations), the collinear libration points $\left(L_{1}-L_{3}\right)$ are unstable, while the equilateral libration points $\left(L_{4}\right.$ and $\left.L_{5}\right)$ are stable [50]. For an unstable equilibrium point, unless an object is placed at exactly the correct point (and there are no outside perturbing forces), the object will not remain near the equilibrium point. Since exact placement is impossible, in practice libration point missions are designed such that the spacecraft orbits the libration point instead (examples include NASA's recent Genesis Discovery Mission [8] and NASA's proposed James Webb Space Telescope Mission [38]). Furthermore, since perturbing forces are inevitable, routine station keeping correction maneuvers are required to maintain the orbit. However, do to the inherent stability of the equilateral libration points, 
orbits about these points are much more efficient (than about the collinear points) since they naturally require less station keeping maneuvering. For this reason, both the cislunar transfer segment and the circular polar orbit segment use the equilateral libration points for observer location placement. Additionally, in order to illustrate some of the observability issues discussed in Section 4.2.2, the circular polar orbit segment also uses the collinear libration point on the far-side of the Moon $\left(L_{2}\right)$. Since the radial magnitude of a libration point orbit relative to its distance to the Moon is negligible (especially for $L_{4}$ and $L_{5}$ ), for simplicity the exact location of these tracking station observers is assumed to be at the libration point.

The location of the libration point tracking stations is assumed to be well known. This could be accomplished by first "surveying-in" the tracking stations at $L_{4}$ and $L_{5}$ using the Earth-based Deep Space Network (DSN) of tracking antennas. If the $L_{2}$ tracking station is in an orbit that remains blocked by the Moon, the $L_{4}$ and $L_{5}$ tracking stations could be used to survey the $L_{2}$ station. While such an architecture would require significant overhead, it may be beneficial in the long run (if long-term lunar operations are desired). Once surveyed, the architecture would require only occasional use of the DSN to station keep the equilateral libration point tracking stations. Thus, such an architecture not only offers an alternative to the already overworked DSN stations, but also provides access to spacecraft operating on the far-side of the Moon.

The ranges from the libration point tracking stations for the circular 
polar orbit segment have the same cyclical trends exhibited in Figure 2.6. Ranges from the station at $L_{2}$ are approximately $0.645 \times 10^{5} \mathrm{~km}$, while those from the stations at $L_{4}$ and $L_{5}$ have a minimum of about $3.83 \times 10^{5} \mathrm{~km}$ and a maximum of about $3.86 \times 10^{5} \mathrm{~km}$; a difference roughly equal to $2 a_{*}$. Due to the geometric symmetry of the problem, this corresponds to the nodal crossings on each side of the orbit. The geometry is fairly constant during the span of time considered in the analysis of Chapter 4, but as discussed earlier, eventually the Earth-Moon line will rotate such that it is $60^{\circ}$ and then $0^{\circ}$ relative to the node line of the formation. Such configurations quickly demonstrate that the tracking station that is inline with the node line will suffer a loss of visibility when the formation passes directly behind the planet. However, due to the low altitude of the formation, similar reductions in visibility are common for the equilateral libration points for all orientations of the Earth-Moon line.

In particular, the visibility factor for the first orbit of the reference point can be seen in Figure 2.6. The visibility factor is a measure of visibility based on the line of sight analysis presented in Vallado [54]. Defined to be the ratio of the angles $\beta$ and $\beta_{\max }$, the visibility factor is greater than or equal to zero and indicates visibility when less than one. The numerator $\beta$, which is the angle between the $i^{\text {th }}$ tracking station position vector $\mathbf{r}_{s_{i}}$ and the reference point position vector $\mathbf{r}_{*}$, can be computed using the vector inner product, yielding

$$
\beta=\cos ^{-1}\left(\frac{\mathbf{r}_{s_{i}}^{T} \mathbf{r}_{*}}{\left\|\mathbf{r}_{s_{i}}\right\|\left\|\mathbf{r}_{*}\right\|}\right)
$$

The denominator, $\beta_{\max }$, is the maximum angle between two vectors of these 


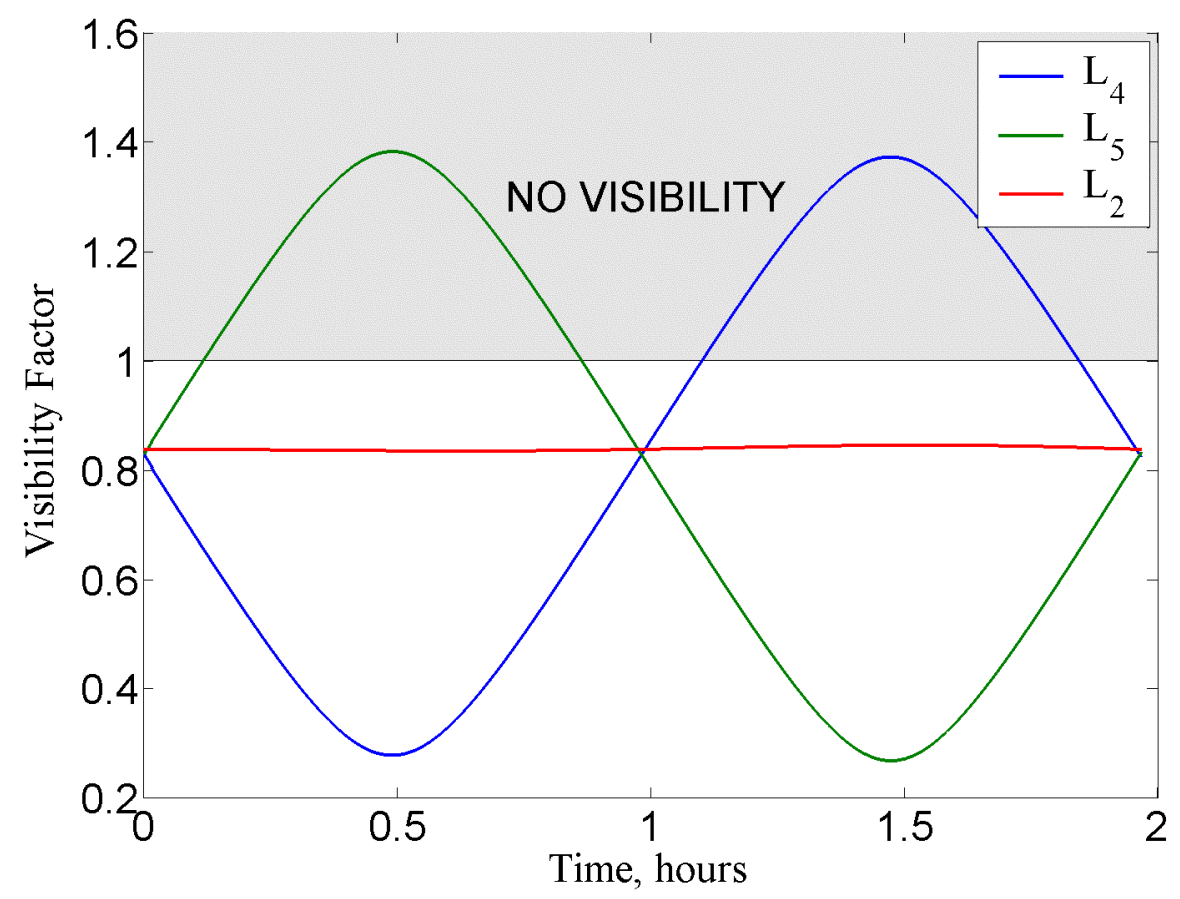

Figure 2.6: Equilateral libration point tracking station visibility.

magnitudes $\left(\left\|\mathbf{r}_{s_{i}}\right\|\right.$ and $\left.\left\|\mathbf{r}_{*}\right\|\right)$ that still allows visibility about a spherical body of radius $R$, in this case $R=R_{\zeta}$. Since this corresponds to when a line connecting two body-centered vectors of these magnitudes is tangential to the surface of the body, $\beta_{\max }$ is the sum of the angles that each vector makes with the resulting normal, such that

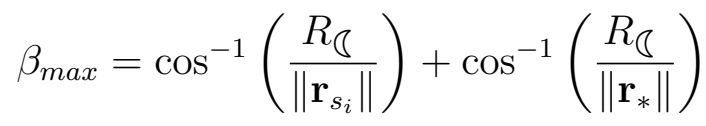

Consequently, if $\beta<\beta_{\max }$ the visibility factor is less than one and the tracking station has visibility of the formation reference point. Since relative to the 
tracking station the formation is practically collocated at the reference point, the visibility for each spacecraft is essentially the same as that of the reference point.

The three intersecting points of visibility factor curves for $L_{4}$ and $L_{5}$ in Figure 2.6 occur when the reference point crosses directly "over" or "under" the Earth-Moon line. At these points the reference point is visible by both equilateral stations since it is "above" and "below" the Moon. As the reference point travels "behind" the Moon however, the corresponding tracking station loses visibility. Although this results in a cyclical loss of visibility to one equilateral station at a time, instead of using only the stations with visibility, it has been assumed that no blackouts occur and all three stations are always used. This has been done in the observability analysis of Chapter 4 to demonstrate some interesting anomalies that result when using three libration point tracking stations, and has been continued elsewhere for continuity and comparison sake.

The cross-link (spacecraft-to-spacecraft) range measurement profile on the other hand, is periodic with the orbit; since in the absence of perturbations the trajectories in Figures 2.4 and 2.5 will repeat every orbital period. In particular, the ranges between spacecraft oscillate in an interval with a minimum of about $3 \mathrm{~km}$ and a maximum of about $26 \mathrm{~km}$, while coplanar spacecraft maintain a distance of $10 \mathrm{~km}$. This large difference in magnitude between station range and cross-link range measurements will be of particular interests in light of the discussion presented in Chapter 5. 
Finally, the geometric simplicity of this formation was intentionally designed to allow for easy visualization of the anomalies described in the observability analysis demonstrated Section 4.2.2.

\subsubsection{System and Measurement Filter Models}

In general, the state to be estimated consists of the body-centered inertial position (r) and velocity ( $\mathbf{v})$ vectors for $p$ spacecraft, such that:

$$
\mathbf{x}=\left[\begin{array}{lll|lll}
\mathbf{r}_{1}^{T} & \cdots & \mathbf{r}_{p}^{T} & \mathbf{v}_{1}^{T} & \cdots & \mathbf{v}_{p}^{T}
\end{array}\right]^{T}
$$

In order to focus on the issues discussed in Chapter 1, extraneous error sources, noise corruptions, and potential numerical instabilities have been avoided whenever possible. For this reason simple Keplerian motion without perturbations is used for both the true environment and the filter dynamical models, where

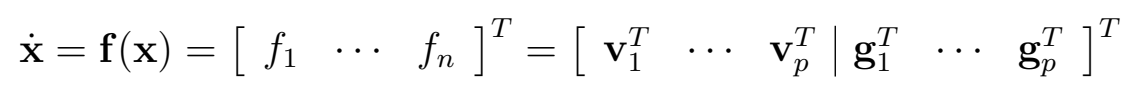

where

$$
\mathbf{g}_{j}=\ddot{\mathbf{r}}_{j}=-\frac{\mu}{\left\|\mathbf{r}_{j}\right\|^{3}} \mathbf{r}_{j}
$$

for $j=1, \ldots, p$ and $\mu$ is the gravitational constant of the central body. Moreover, unless otherwise stated, system noise is not implemented (i.e. $\mathbf{Q}_{\text {spec }}=\mathbf{0}$, see Sections 3.1 and 3.3).

Another reason for choosing the Keplerian dynamical model was to simplify the derivation and computation of the Jacobian and Hessian matrices 
that will later be required by the Kalman filter (see Chapter 3). Using Eqs. (2.17) and (2.18), the system dynamics matrix can be partitioned as,

$$
\frac{\partial \mathbf{f}}{\partial \mathbf{x}}=\left[\begin{array}{c|c}
\mathbf{0} & \mathbf{I} \\
\hline \mathbf{G} & \mathbf{0}
\end{array}\right]_{n \times n} \quad, \quad \mathbf{G}=\left[\begin{array}{ccc}
\mathbf{G}_{1} & \mathbf{0} & \mathbf{0} \\
\mathbf{0} & \ddots & \mathbf{0} \\
\mathbf{0} & \mathbf{0} & \mathbf{G}_{p}
\end{array}\right]
$$

where for $j=1, \ldots, p$,

$$
\mathbf{G}_{j}:=\frac{\partial \mathbf{g}_{j}}{\partial \mathbf{r}_{j}}=\frac{\mu}{\left\|\mathbf{r}_{j}\right\|^{5}}\left(3 \mathbf{r}_{j} \mathbf{r}_{j}^{T}-\left\|\mathbf{r}_{j}\right\|^{2} \mathbf{I}\right)
$$

The Hessian can be similarly partitioned as

$$
\frac{\partial^{2} f_{i}}{\partial \mathbf{x}^{2}}=\left[\begin{array}{c|c}
\boldsymbol{\Delta}_{f_{i}} & \mathbf{0} \\
\hline \mathbf{0} & \mathbf{0}
\end{array}\right]_{n \times n}
$$

The $n / 2 \times n / 2$ matrix $\boldsymbol{\Delta}_{f_{i}}$ is $\mathbf{0}$ for $i \leq n / 2$, and sparse otherwise. In particular, for $i=n / 2+3(j-1)+k>n / 2$ the matrix $\boldsymbol{\Delta}_{f_{i}}$ has $\mathbf{0}$ submatrices everywhere except for the $3 \times 3$ nonzero submatrix in rows and columns $3 j-2,3 j-1,3 j$ denoted $\mathbf{G}_{j k}^{\prime}$ where

$$
\mathbf{G}_{j k}^{\prime}:=\frac{\partial^{2} g_{j_{k}}}{\partial \mathbf{r}_{j}^{2}}=\frac{3 \mu}{\left\|\mathbf{r}_{j}\right\|^{5}}\left(\mathbf{r}_{j} \mathbf{I}_{k}^{T}+\mathbf{I}_{k} \mathbf{r}_{j}^{T}+r_{j_{k}} \mathbf{I}-5 r_{j_{k}} \frac{\mathbf{r}_{j} \mathbf{r}_{j}^{T}}{\left\|\mathbf{r}_{j}\right\|^{2}}\right)
$$

The subscript $j$ refers to the spacecraft number $(j=1, \ldots, p)$, the subscript $k$ refers to the $x, y$, or, $z$ component of $\mathbf{v}_{j}$ or $\mathbf{g}_{j}(k=1,2,3$, respectively) corresponding to $f_{i}$, and $\mathbf{I}_{k}$ refers to the $k^{\text {th }}$ column of the $3 \times 3$ identity matrix $\mathbf{I}$.

For comparison, whenever a formation is examined two measurement scenarios are considered. The first scenario involves the use of only the iner- 
tial $^{1}$ range measurements from the three libration point tracking stations to each spacecraft $\left(m=m_{\text {in }}=3 p\right)$. The second scenario employs inertial range measurements with the addition of $m_{\text {rel }}$ relative range measurements between each spacecraft $\left(m=m_{\text {in }}+m_{\text {rel }} \text { and } m_{r e l}=p(p-1) / 2\right)^{2}$. Thus, the measurement model for $k=1, \ldots, m$ where $1 \leq k \leq m_{\text {in }}$ refers to an inertial range measurement and $k>m_{\text {in }}$ refers to a relative range measurement can be constructed as,

$$
\mathbf{h}(\mathbf{x})=\left[\begin{array}{lll}
h_{1} & \cdots & h_{m}
\end{array}\right]^{T} \quad, \quad h_{k}=\rho_{i j}=\left\|\boldsymbol{\rho}_{i j}\right\|
$$

where

$$
\boldsymbol{\rho}_{i j}= \begin{cases}\mathbf{r}_{j}-\mathbf{r}_{s_{i}} & 1 \leq k \leq m_{i n} \\ \mathbf{r}_{j}-\mathbf{r}_{i} & k>m_{\text {in }}\end{cases}
$$

The subscript $j$ refers to the primary spacecraft number, while the subscript $i$ refers to the station number (for inertial measurements) or the secondary spacecraft number (for relative measurements) that is tracking the primary. Consequently, $\mathbf{r}_{s_{i}}$ refers to the position vector of the $i^{\text {th }}$ tracking station, where $i$ corresponds to the station number of $L_{4}, L_{5}$, or $L_{2}$.

To avoid potential numerical instabilities and to ensure that the complications when fusing relative measurements are not a result of the previously

\footnotetext{
${ }^{1}$ Practically speaking station range measurements are also relative in that they are taken relative to a non-inertial observer. However, for discussion purposes, the term "inertial range measurement" is used to refer to a range measurement taken from an observer with a known ephemeris in the inertial frame; where as the term "relative range measurement" is reserved for a range measurement taken between spacecraft contained in the estimated state.

${ }^{2}$ When a single cluster of spacecraft $(p=1)$ is examined during the cislunar transfer segment, only the two equilateral stations are used and $m=2$.
} 
mentioned measurement accuracy paradox, all inertial and relative range measurements are simulated using an identical measurement noise, which has a standard deviation of $\sigma_{\rho}=5 \mathrm{~m}$. Likewise, unless otherwise stated, the filter uses the same corresponding measurement noise covariance (i.e. $\mathbf{R}=\sigma_{\rho}^{2} \mathbf{I}$, see Sections 3.1 and 3.4). All measurements are available simultaneously at equally spaced intervals, and unless otherwise stated, the measurements are processed simultaneously the instant they become available. The cislunar transfer segment uses a measurement frequency of one hour, while the circular polar orbit segment uses a frequency of one minute. Finally, since designing the logistics required to implement such a filter is not the objective of this dissertation, it has been assumed here that the spacecraft can instantly exchange serial data messages containing the inertial and relative measurement information of the other spacecraft in the formation.

With the measurement model defined in Eq. (2.24), the derivation and computation of the Jacobian and Hessian matrices, which will later be required by the Kalman filter, can be obtained. In particular, reexamining Eq. (2.17) and noting that the measurements are not functions of velocity, the measurement mapping matrix can be partitioned as,

$$
\frac{\partial \mathbf{h}}{\partial \mathbf{x}}=[\boldsymbol{\Theta} \mid \mathbf{0}]_{m \times n} \quad \text { where } \quad \boldsymbol{\Theta}:=\left[\begin{array}{lll}
\frac{\partial \mathbf{h}}{\partial \mathbf{r}_{1}} & \cdots & \frac{\partial \mathbf{h}}{\partial \mathbf{r}_{p}}
\end{array}\right]
$$

which has nonzero components

$$
\frac{\partial h_{k}}{\partial \mathbf{r}_{j}}=\frac{\partial \rho_{i j}}{\partial \mathbf{r}_{j}}=+\frac{\boldsymbol{\rho}_{i j}^{T}}{\rho_{i j}} \quad \text { and } \quad \frac{\partial h_{k}}{\partial \mathbf{r}_{i}}=\frac{\partial \rho_{i j}}{\partial \mathbf{r}_{i}}=\left\{\begin{array}{rl}
\mathbf{0}^{T} & 1 \leq k \leq m_{i n} \\
-\frac{\boldsymbol{\rho}_{i j}^{T}}{\rho_{i j}} & k>m_{i n}
\end{array}\right.
$$


and zero components elsewhere. Partitioning the corresponding Hessian results in

$$
\frac{\partial^{2} h_{k}}{\partial \mathbf{x}^{2}}=\left[\begin{array}{c|c}
\boldsymbol{\Delta}_{h_{k}} & \mathbf{0} \\
\hline \mathbf{0} & \mathbf{0}
\end{array}\right]_{n \times n}
$$

The $n / 2 \times n / 2$ matrix $\boldsymbol{\Delta}_{h_{k}}$ has $\mathbf{0}$ submatrices everywhere except for the $3 \times 3$ nonzero submatrix in rows and columns $3 j-2,3 j-1,3 j$ denoted $\Theta_{i j}^{\prime}$,

$$
\boldsymbol{\Theta}_{i j}^{\prime}:=\frac{\partial^{2} \rho_{i j}}{\partial \mathbf{r}_{j}^{2}}=\frac{1}{\rho_{i j}^{3}}\left(\rho_{i j}^{2} \mathbf{I}-\boldsymbol{\rho}_{i j} \boldsymbol{\rho}_{i j}^{T}\right)
$$

The subscript $j$ refers to the primary spacecraft number, while the subscript $i$ refers to the station number (for inertial measurements) or the secondary spacecraft number (for relative measurements) that is tracking the primary. Finally, for relative measurements $\left(k>m_{i n}\right)$ the submatrix $\boldsymbol{\Theta}_{i j}^{\prime}$ appears four times due to the additional rows and columns $3 i-2,3 i-1,3 i$, except the two off diagonal submatrices are negative $\left(-\Theta_{i j}^{\prime}\right)$. 


\section{Chapter 3}

\section{Filter Algorithms}

Two navigation algorithms are considered. The first algorithm is the continuous-discrete extended Kalman filter (EKF) (see Gelb [19] for more details). The EKF is an extension of the optimal linear Kalman filter. The Kalman filter is a recursive data processing algorithm that uses measurement data and prior knowledge about the system and measurement models to produce an estimate of the desired state variables such that the mean squared estimate error is minimized. When the system and measurement models are linear and the system and measurement noise are modeled as white Gaussian random processes the linear Kalman filter is the optimal filter [39]. No other filter structure, such as a nonlinear filter, provides better state estimates. While the white noise assumptions can often be justified, for a typical orbital mechanics problem, the system and measurement models cannot be accurately described as linear. The EKF accommodates these nonlinearities by using first-order Taylor series expansions to linearize the models about the current state estimate. Thus, given the continuous nonlinear equations of motion and discrete measurements, the EKF provides the minimum variance mean square estimate of the state at each instance of time. 
The second navigation algorithm considered is a variation of the EKF known as the modified Gaussian second-order filter (GSF) (see Maybeck [40] for more details). The GSF is an EKF that incorporates second-order terms of the Taylor series expansions in the linearization process. This is accomplished by assuming the conditional density is Gaussian and that third and higherorder odd central moments are zero, while fourth and higher-order even central moments can be expressed in terms of the covariance. The GSF is implemented in Section 5.3. However, since the GSF can easily be reduced to the EKF by setting the second-order terms equal to zero, the GSF algorithm is presented

here and the EKF follows directly. Finally, when possible without loss of clarity, functional dependencies on the state and time have been suppressed for compactness and readability.

\subsection{General Models}

The system model as a function of the state $\mathbf{x} \in \mathbb{R}^{n}$ has the form,

$$
\dot{\mathbf{x}}(t)=\mathbf{f}(\mathbf{x}(t), t)+\mathbf{w}(t)
$$

with

$$
E[\mathbf{w}(t)]=\mathbf{0} \quad \text { and } \quad E\left[\mathbf{w}(t) \mathbf{w}^{T}(\tau)\right]=\mathbf{Q}_{\text {spec }} \delta(t-\tau)
$$

where $\delta(t-\tau)$ is Dirac delta function defined by Eq. (2.1). The nonlinear dynamical model $\mathbf{f}$ is assumed to be sufficiently differentiable. The process noise, denoted $\mathbf{w}(t)$, is modeled as a zero-mean white noise process with constant power spectral density $\mathbf{Q}_{\text {spec }} \in \mathbb{R}^{n \times n}$. The constant power spectral density 
implies that the stochastic process has infinite variance. This is an idealized concept that is not physically realizable, however it does serve as a useful mechanism for modeling system uncertainties and disturbances. The matrix $\mathbf{Q}_{\text {spec }} \geq \mathbf{0}$ is a filter input. The noise $\mathbf{w}(t)$ represents those disturbances that are unmodeled because they are unknown, as well as those intentionally excluded due to model complexity or computational limitations. Consequently, in simulation, the true environment dynamics are not simulated by integrating the filter dynamical model plus noise as Eq. (3.1) would suggest, but instead by integrating an environment dynamical model that is of a higher fidelity than the filter dynamical model. Eq. (3.1) is the model employed in the EKF design.

Similarly, the measurement model for the measurement vector $\mathbf{z} \in \mathbb{R}^{m}$ has the form,

$$
\mathbf{z}\left(t_{d}\right)=\mathbf{h}\left(\mathbf{x}\left(t_{d}\right)\right)+\mathbf{u}\left(t_{d}\right)
$$

with

$$
E\left[\mathbf{u}\left(t_{d}\right)\right]=\mathbf{0} \quad \text { and } \quad E\left[\mathbf{u}\left(t_{c}\right) \mathbf{u}^{T}\left(t_{d}\right)\right]=\mathbf{R}\left(t_{d}\right) \delta_{c d}
$$

where $\delta_{c d}$ is the Kronecker delta defined by Eq. (2.2). The nonlinear measurement model $\mathbf{h}$ is assumed to be sufficiently differentiable. The measurement noise, denoted $\mathbf{u}\left(t_{d}\right)$, is modeled as a zero-mean white noise sequence with noise covariance $\mathbf{R} \in \mathbb{R}^{m \times m}$. The matrix $\mathbf{R}$ is a filter input.

It is also assumed that the system and measurement noise are uncor- 
related in time, that is,

$$
E\left[\mathbf{w}(t) \mathbf{u}^{T}\left(t_{d}\right)\right]=\mathbf{0} \quad \forall \quad t, t_{d}
$$

The state estimate error $\mathbf{e}$ is defined as

$$
\mathbf{e}(t)=\mathbf{x}(t)-\hat{\mathbf{x}}(t)
$$

with

$$
E[\mathbf{e}(t)]=\mathbf{0} \quad \text { and } \quad E\left[\mathbf{e}(t) \mathbf{e}^{T}(t)\right]=\mathbf{P}(t)
$$

and the initial state estimate $\hat{\mathbf{x}}\left(t_{0}\right)$ is such that the initial state error $\mathbf{e}\left(t_{0}\right)$ is zero-mean with covariance $\mathbf{P}\left(t_{0}\right)$. Since the computed state error covariance is only an approximation to the true state error covariance (in the nonlinear setting), the actual filter performance must be verified through Monte Carlo analysis.

\subsection{Model Linearization}

An essential element of the linearization process is the use of Taylor series expansions to obtain a linear representation of the nonlinear models. In the design of the EKF and GSF, the linearization process retains up to first and second-order terms, respectively, of the Taylor series expansion about the current state estimate. To illustrate this, first consider the expansion of an arbitrary (infinitely continuously differentiable) scalar function $y(x) \in \mathbb{R}$, where $x \in \mathbb{R}$, about a reference point $\hat{x}[20]$ :

$$
y(x)=\sum_{k=1}^{\infty} \frac{y^{(k)}(\hat{x})}{k !}(x-\hat{x})^{k}=y(\hat{x})+y^{\prime}(\hat{x})(x-\hat{x})+\frac{y^{\prime \prime}(\hat{x})}{2}(x-\hat{x})^{2}+\ldots
$$


where $y^{\prime}(\hat{x}):=\partial y /\left.\partial x\right|_{x=\hat{x}}, y^{\prime \prime}(\hat{x}):=\partial^{2} y /\left.\partial x^{2}\right|_{x=\hat{x}}$, and so on. Retaining terms up to $k=2$, yields a second-order approximation of $y(x)$ for $x$ values near $\hat{x}$.

In order to use this concept to develop the filter equations, it is necessary to consider that $\mathbf{y}(\mathbf{x})$ is now an $m$-dimensional vector function, where $\mathbf{x}$ is an $n$-dimensional vector. To make the problem more manageable, only one scalar component of $\mathbf{y}$ is consider at a time, and the expansion of the $i^{\text {th }}$ component becomes,

$$
y_{i}(\mathbf{x})=y_{i}(\hat{\mathbf{x}})+\mathbf{Y}_{i}(\mathbf{x}-\hat{\mathbf{x}})+\frac{1}{2}(\mathbf{x}-\hat{\mathbf{x}})^{T} \mathbf{Y}_{i}^{\prime}(\mathbf{x}-\hat{\mathbf{x}})+\ldots
$$

where the Jacobian $\mathbf{Y}_{i}$ and Hessian $\mathbf{Y}_{i}^{\prime}$ are defined as

$$
\mathbf{Y}_{i}(\hat{\mathbf{x}}):=\left.\frac{\partial y_{i}}{\partial \mathbf{x}}\right|_{\mathbf{x}=\hat{\mathbf{x}}} \in \mathbb{R}^{1 \times n} \quad \text { and } \quad \mathbf{Y}_{i}^{\prime}(\hat{\mathbf{x}}):=\left.\frac{\partial^{2} y_{i}}{\partial \mathbf{x}^{2}}\right|_{\mathbf{x}=\hat{\mathbf{x}}} \in \mathbb{R}^{n \times n}
$$

with the shorthand notation $\partial^{2} y_{i} / \partial \mathbf{x}^{2} \equiv \partial\left(\partial y_{i} / \partial \mathbf{x}\right)^{T} / \partial \mathbf{x}$. It is also useful to note that since the terms of Eq. (3.9) are scalars, the properties of the trace operation can be used to rewrite the quadratic term as,

$$
\frac{1}{2}(\mathbf{x}-\hat{\mathbf{x}})^{T} \mathbf{Y}_{i}^{\prime}(\mathbf{x}-\hat{\mathbf{x}})=\frac{1}{2} \operatorname{trace}\left(\mathbf{e}^{T} \mathbf{Y}_{i}^{\prime} \mathbf{e}\right)=\frac{1}{2} \operatorname{trace}\left(\mathbf{Y}_{i}^{\prime} \mathbf{e e}^{T}\right)
$$

Thus, by considering $\mathbf{y}=\mathbf{f}$ or $\mathbf{y}=\mathbf{h}$ the dynamical and measurement models, respectively, can be approximated up to second-order using Eq. (3.9).

The filter algorithm architecture has two phases: a propagation phase and an update phase. The algorithms (both the EKF and GSF) are hybrid continuous-discrete systems wherein the measurements are processed at discrete times (as they become available) and otherwise the state estimate is 
maintained by employing a model-based propagation scheme. Representing the state estimate as $\hat{\mathbf{x}} \in \mathbb{R}^{n}$, at time $t_{d}$ before a measurement is processed, the state estimate is denoted by $\hat{\mathbf{x}}\left(t_{d}^{-}\right)$. After the measurement is processed and the state estimate is updated accordingly, it is denoted by $\hat{\mathbf{x}}\left(t_{d}^{+}\right)$. During the time interval $t_{d}$ to $t_{d+1}$, the state estimate is propagated according to a model of the system, and at $t_{d+1}$ before the measurement is incorporated, the state estimate is $\hat{\mathbf{x}}\left(t_{d+1}^{-}\right)$.

In particular, the first-order state estimate is obtained via integration of

$$
\dot{\hat{\mathbf{x}}}(t)=\mathbf{f}(\hat{\mathbf{x}}(t)) \quad, \quad t_{d-1} \leq t \leq t_{d}
$$

with $\hat{\mathbf{x}}\left(t_{d-1}\right)=\hat{\mathbf{x}}\left(t_{d-1}^{+}\right)$. Then, differentiating the state estimate error, $\mathbf{e}$, in Eq. (3.6), the continuous linearized error dynamics (to first-order) can be written as,

$$
\begin{aligned}
\dot{\mathbf{e}}(t) & =\dot{\mathbf{x}}(t)-\dot{\hat{\mathbf{x}}}(t)=\mathbf{f}(\mathbf{x}(t))-\mathbf{f}(\hat{\mathbf{x}}(t))+\mathbf{w}(t) \\
& \approx \mathbf{F}(\hat{\mathbf{x}}(t)) \mathbf{e}(t)+\mathbf{w}(t)
\end{aligned}
$$

where using Eq. (3.10) with $\mathbf{y}=\mathbf{f}$ the so-called system dynamics matrix is defined as:

$$
\mathbf{F}(\hat{\mathbf{x}}(t)):=\left.\frac{\partial \mathbf{f}}{\partial \mathbf{x}}\right|_{\mathbf{x}=\hat{\mathbf{x}}(t)} \in \mathbb{R}^{n \times n}
$$

The discrete time solution to the linearized differential equation in Eq. (3.13) is

$$
\mathbf{e}\left(t_{d}\right)=\boldsymbol{\Phi}\left(t_{d}, t_{d-1}\right) \mathbf{e}\left(t_{d-1}\right)+\mathbf{w}\left(t_{d}\right)
$$

where $\boldsymbol{\Phi}\left(t_{d}, t_{d-1}\right)$ is obtained by integrating

$$
\dot{\boldsymbol{\Phi}}\left(t, t_{d-1}\right)=\mathbf{F}(\hat{\mathbf{x}}(t)) \boldsymbol{\Phi}\left(t, t_{d-1}\right) \quad, \quad t_{d-1} \leq t \leq t_{d}
$$


with $\boldsymbol{\Phi}\left(t_{d-1}, t_{d-1}\right)=\mathbf{I}$. The sequence $\mathbf{w}\left(t_{d}\right)$ is formally defined from the white noise process $\mathbf{w}(t)$ as,

$$
\mathbf{w}\left(t_{d}\right):=\int_{t_{d-1}}^{t_{d}} \boldsymbol{\Phi}\left(t_{d}, \tau\right) \mathbf{w}(\tau) d \tau
$$

and has the properties,

$$
E\left[\mathbf{w}\left(t_{d}\right)\right]=\mathbf{0} \quad \text { and } \quad E\left[\mathbf{w}\left(t_{c}\right) \mathbf{w}^{T}\left(t_{d}\right)\right]=\mathbf{Q}\left(t_{d}\right) \delta_{c d}
$$

where $\delta_{c d}$ is the Kronecker delta defined by Eq. (2.2).

A similar linearization process is followed for the nonlinear measurement model. At the time a measurement is available, there are two pieces of information: the measurement itself and an estimate of the expected measurement. The difference between the two, known as the measurement residual, is defined as

$$
\boldsymbol{\epsilon}\left(t_{d}\right):=\mathbf{z}\left(t_{d}\right)-\mathbf{h}\left(\hat{\mathbf{x}}\left(t_{d}^{-}\right)\right)
$$

where $\hat{\mathbf{x}}\left(t_{d}^{-}\right)$is the state estimate before the state update. Then, with the measurement model as in Eq. (3.3) it follows that the discrete linearized measurement residual (to first-order) can be written as,

$$
\begin{aligned}
\boldsymbol{\epsilon}\left(t_{d}\right) & =\mathbf{h}\left(\mathbf{x}\left(t_{d}\right)\right)-\mathbf{h}\left(\hat{\mathbf{x}}\left(t_{d}^{-}\right)\right)+\mathbf{u}\left(t_{d}\right) \\
& \approx \mathbf{H}\left(\hat{\mathbf{x}}\left(t_{d}^{-}\right)\right) \mathbf{e}\left(t_{d}\right)+\mathbf{u}\left(t_{d}\right)
\end{aligned}
$$

where using Eq. (3.10) with $\mathbf{y}=\mathbf{h}$ the so-called measurement mapping matrix is defined as:

$$
\mathbf{H}\left(\hat{\mathbf{x}}\left(t_{d}^{-}\right)\right):=\left.\frac{\partial \mathbf{h}}{\partial \mathbf{x}}\right|_{\mathbf{x}=\hat{\mathbf{x}}\left(t_{d}^{-}\right)} \in \mathbb{R}^{m \times n}
$$




\subsection{State and State Error Covariance Propagation}

The second-order state estimate is propagated between measurements using the dynamical model $\mathbf{f}$ such that

$$
\dot{\hat{\mathbf{x}}}(t)=\mathbf{f}(\hat{\mathbf{x}}(t))+\mathbf{b}_{f}(t) \quad, \quad t_{d-1} \leq t \leq t_{d}
$$

where $\mathbf{b}_{f}$ is the bias compensation term resulting from the inclusion of secondorder terms. Consequently, if second-order terms are ignored $\mathbf{b}_{f}=\mathbf{0}$ and Eq. (3.22) reduces to the standard state estimate propagation equation used by the EKF. The $i^{t h}$ component of the bias term, denoted by $b_{f_{i}}$, can be written as a function of the state error covariance $\mathbf{P}$ (defined in Eq. (3.7)) as

$$
b_{f_{i}}(t)=\frac{1}{2} \operatorname{trace}\left(\mathbf{F}_{i}^{\prime}(\hat{\mathbf{x}}(t)) \mathbf{P}(t)\right) \quad, \quad i=1, \ldots, n
$$

where $\mathbf{F}_{i}^{\prime}$ is the Hessian of the $i^{\text {th }}$ component of the dynamical model $f_{i}$ with respect to the state and evaluated at the current state estimate. Using Eq. (3.10) with $\mathbf{y}=\mathbf{f}$, the matrix $\mathbf{F}_{i}^{\prime}$ is defined as

$$
\mathbf{F}_{i}^{\prime}(\hat{\mathbf{x}}(t)):=\left.\frac{\partial^{2} f_{i}}{\partial \mathbf{x}^{2}}\right|_{\mathbf{x}=\hat{\mathbf{x}}(t)}
$$

The state error covariance is propagated between measurements by directly integrating

$$
\dot{\mathbf{P}}(t)=\mathbf{F}(\hat{\mathbf{x}}(t)) \mathbf{P}(t)+\mathbf{P}(t) \mathbf{F}^{T}(\hat{\mathbf{x}}(t))+\mathbf{Q}_{\text {spec }} \quad, \quad t_{d-1} \leq t \leq t_{d}
$$

with initial condition $\mathbf{P}\left(t_{d-1}\right)=\mathbf{P}\left(t_{d-1}^{+}\right)$, or by using the state transition matrix and the process noise covariance $\mathbf{Q}\left(t_{d}\right)$, such that

$$
\mathbf{P}\left(t_{d}^{-}\right)=\boldsymbol{\Phi}\left(t_{d}, t_{d-1}\right) \mathbf{P}\left(t_{d-1}^{+}\right) \boldsymbol{\Phi}^{T}\left(t_{d}, t_{d-1}\right)+\mathbf{Q}\left(t_{d}\right)
$$


The latter method requires the integration of the process noise covariance via

$$
\dot{\mathbf{Q}}(t)=\mathbf{F}(\hat{\mathbf{x}}(t)) \mathbf{Q}(t)+\mathbf{Q}(t) \mathbf{F}^{T}(\hat{\mathbf{x}}(t))+\mathbf{Q}_{\text {spec }} \quad, \quad t_{d-1} \leq t \leq t_{d}
$$

with $\mathbf{Q}\left(t_{d-1}\right)=\mathbf{0}$. The state transition matrix has many useful applications including state observability analysis. Consequently, the filters implemented in this dissertation use the state transition matrix to propagate the state error covariance.

\subsection{State and State Error Covariance Update}

The state estimate and state error covariance are propagated until a measurement becomes available at time $t_{d}$. At $t_{d}$, the state estimate and state error covariance are updated by incorporating the measurement. As described in Section 2.1, values immediately prior to the measurement update are referred to as a priori values and are denoted with a superscript "-". Conversely, values immediately after the measurement update are referred to as a posteriori values and are denoted with a superscript "+". Thus, the state estimate update at time $t_{d}$ can be written as

$$
\hat{\mathbf{x}}\left(t_{d}^{+}\right)=\hat{\mathbf{x}}\left(t_{d}^{-}\right)+\mathbf{K}\left(t_{d}\right) \boldsymbol{\epsilon}\left(t_{d}\right)
$$

where $\mathbf{K}$ is the Kalman gain, $\boldsymbol{\epsilon}$ is the second-order measurement residual defined as,

$$
\boldsymbol{\epsilon}\left(t_{d}\right):=\mathbf{z}\left(t_{d}\right)-\mathbf{h}\left(\hat{\mathbf{x}}\left(t_{d}^{-}\right)\right)-\mathbf{b}_{h}\left(t_{d}\right)
$$

and $\mathbf{b}_{h}$ is the bias compensation term resulting from the inclusion of secondorder terms. The term $\mathbf{b}_{h}$ is absent in the first-order (EKF) formulation. The 
$k^{\text {th }}$ component of the bias term, denoted by $b_{h_{k}}$, can be written as a function of the state error covariance $\mathbf{P}$ as

$$
b_{h_{k}}\left(t_{d}\right)=\frac{1}{2} \operatorname{trace}\left(\mathbf{H}_{k}^{\prime}\left(\hat{\mathbf{x}}\left(t_{d}^{-}\right)\right) \mathbf{P}\left(t_{d}^{-}\right)\right) \quad, \quad k=1, \ldots, m
$$

where $\mathbf{H}_{k}^{\prime}$ is the Hessian of the $k^{t h}$ component of the measurement model $h_{k}$ with respect to the state and evaluated at the a priori state estimate. Using Eq. (3.10) with $\mathbf{y}=\mathbf{h}$, the matrix $\mathbf{H}_{k}^{\prime}$ is defined as

$$
\mathbf{H}_{k}^{\prime}\left(\hat{\mathbf{x}}\left(t_{d}^{-}\right)\right):=\left.\frac{\partial^{2} h_{k}}{\partial \mathbf{x}^{2}}\right|_{\mathbf{x}=\hat{\mathbf{x}}\left(t_{d}^{-}\right)}
$$

The Kalman gain at time $t_{d}$ is given by

$$
\mathbf{K}\left(t_{d}\right)=\mathbf{P}\left(t_{d}^{-}\right) \mathbf{H}^{T}\left(\hat{\mathbf{x}}\left(t_{d}^{-}\right)\right) \mathbf{W}^{-1}\left(t_{d}\right)
$$

where $\mathbf{W}$ is the measurement residual covariance defined as

$$
\mathbf{W}\left(t_{d}\right):=\mathbf{H}\left(\hat{\mathbf{x}}\left(t_{d}^{-}\right)\right) \mathbf{P}\left(t_{d}^{-}\right) \mathbf{H}^{T}\left(\hat{\mathbf{x}}\left(t_{d}^{-}\right)\right)+\mathbf{R}\left(t_{d}\right)+\mathbf{B}_{h}\left(t_{d}\right)
$$

and $\mathbf{B}_{h}$ is the additional covariance term resulting from the inclusion of secondorder terms. The $j k^{t h}$ component $(j=1, \ldots, m)$ of the covariance term $B_{h_{j k}}$ can be written as a function of the state error covariance $\mathbf{P}$ as follows:

$$
B_{h_{j k}}\left(t_{d}\right)=\frac{1}{2} \operatorname{trace}\left(\mathbf{H}_{j}^{\prime}\left(\hat{\mathbf{x}}\left(t_{d}^{-}\right)\right) \mathbf{P}\left(t_{d}^{-}\right) \mathbf{H}_{k}^{\prime}\left(\hat{\mathbf{x}}\left(t_{d}^{-}\right)\right) \mathbf{P}\left(t_{d}^{-}\right)\right)
$$

Once the Kalman gain has been computed the state error covariance is also updated at time $t_{d}$ using the Joseph formula:

$$
\begin{aligned}
\mathbf{P}\left(t_{d}^{+}\right)= & {\left[\mathbf{I}-\mathbf{K}\left(t_{d}\right) \mathbf{H}\left(\hat{\mathbf{x}}\left(t_{d}^{-}\right)\right)\right] \mathbf{P}\left(t_{d}^{-}\right)\left[\mathbf{I}-\mathbf{K}\left(t_{d}\right) \mathbf{H}\left(\hat{\mathbf{x}}\left(t_{d}^{-}\right)\right)\right]^{T} } \\
& +\mathbf{K}\left(t_{d}\right) \mathbf{R}\left(t_{d}\right) \mathbf{K}^{T}\left(t_{d}\right)
\end{aligned}
$$


Although by combining Eqs. (3.32) and (3.35), the Joseph formula is reduced to the more compact and computationally efficient equation,

$$
\mathbf{P}\left(t_{d}^{+}\right)=\left[\mathbf{I}-\mathbf{K}\left(t_{d}\right) \mathbf{H}\left(\hat{\mathbf{x}}\left(t_{d}^{-}\right)\right)\right] \mathbf{P}\left(t_{d}^{-}\right)
$$

this form of the update is usually undesirable because it has poor numerical properties. The state error covariance is re-symmetrized after the update.

If the second-order terms are ignored, that is, if $\mathbf{b}_{h}=\mathbf{0}$ and $\mathbf{B}_{h}=\mathbf{0}$, then Eqs. (3.28), (3.32), and (3.35) reduce to the standard update equations used by the EKF. Applying this result to Eq. (3.29), as in Eq. (3.19), the EKF residual is the difference between the actual measurement and the modeled measurement evaluated at the a priori state estimate at the time of the measurement. When the filter is operating properly, the residual is expected to be a zero-mean, white noise sequence with covariance W. In other words,

$$
E\left[\boldsymbol{\epsilon}\left(t_{d}\right)\right]=\mathbf{0} \quad \text { and } \quad E\left[\boldsymbol{\epsilon}\left(t_{c}\right) \boldsymbol{\epsilon}^{T}\left(t_{d}\right)\right]=\mathbf{W}\left(t_{d}\right) \delta_{c d}
$$

where $\delta_{c d}$ is the Kronecker delta defined by Eq. (2.2). In fact, by examining Eqs. (3.19) and (3.33) for the EKF, it can be shown that in the theoretical case when the state estimate is perfect $(\hat{\mathbf{x}}=\mathbf{x}, \mathbf{P}=0)$, then it follows that $\boldsymbol{\epsilon}=\mathbf{u}$ and $\mathbf{W}=\mathbf{R}$.

\subsection{Numerical Issues}

Alternative update algorithms have been developed to avoid the computational and numerical issues associated with the required matrix inversion 
of the residual covariance. The size of the matrix to be inverted is $m \times m$, where $m$ is the dimension of the measurement vector. Consequently, when processing a scalar measurement the algorithm only requires the inversion of a scalar. The vector measurement case can be reduced to the scalar measurement case by considering the simultaneous measurement components to occur consecutively over an infinitesimal span of time. Applying this method results in a modified algorithm described in Chapter 5. It is important to note that this method causes the order in which measurements are processed to become significant. In particular, if the state estimate is updated as each scalar measurement is processed, each subsequent linearization of the measurement model is about a more accurate state estimate. This is an interesting detail that will be exploited in Section 5.2.1.

There are additional ways to avoid computing the matrix inverse without artificially inferring importance to the order in which measurements are processed. By rearranging Eq. (3.32), notice that the update does not explicitly require the computation of the inverse of the residual covariance matrix, but only the solution $\mathbf{K}$ of the following linear system: $\mathbf{K W}=\mathbf{P}^{-} \mathbf{H}^{T}$. One method for solving for $\mathbf{K}$ is to triangularize the system and then use forward and/or backward substitution to obtain the solution. In particular, since the residual covariance matrix is symmetric positive definite, Cholesky decomposition [21] can be used to factor $\mathbf{W}$ into the product of a lower-triangular matrix $\mathbf{L}$ and its transpose, such that $\mathbf{W}=\mathbf{L} \mathbf{L}^{T}$. Then, since $\mathbf{K} \mathbf{L} \mathbf{L}^{T}=\mathbf{P}^{-} \mathbf{H}^{T}$ and $\mathbf{L}^{T}$ is an upper-triangular matrix, the matrix $\mathbf{K L}$ can quickly be solved for 
using forward substitution. Similarly, with KL known and L being a lowertriangular matrix, $\mathbf{K}$ can be solved for using backward substitution.

To ensure numerical stability, unless otherwise stated, the filters implemented in this dissertation employ the matrix form of the equations with Cholesky decomposition to obtain the Kalman gain, and the Joseph formula to update the state error covariance.

\subsection{Algorithm Summary}

The EKF and GSF algorithms are summarized in Table 3.1. Functional dependencies on the state and time have been suppressed for compactness and readability. For example, the notation $\hat{\mathbf{x}}^{+}$and $\mathbf{P}^{-}$denotes $\hat{\mathbf{x}}\left(t_{d}^{+}\right)$and $\mathbf{P}\left(t_{d}^{-}\right)$, respectively.

Table 3.1: Filter Algorithm Summary

\begin{tabular}{|c|c|c|c|}
\hline & & $\mathrm{EKF}$ & GSF \\
\hline \multirow{2}{*}{$\begin{array}{l}\text { Propagation } \\
t \in\left[t_{d-1}, t_{d}\right]\end{array}$} & & $\dot{\hat{\mathbf{x}}}=\mathbf{f}(\hat{\mathbf{x}})$ & $\dot{\hat{\mathbf{x}}}=\mathbf{f}(\hat{\mathbf{x}})+\mathbf{b}_{f}$ \\
\hline & \multicolumn{3}{|c|}{$\dot{\mathbf{P}}=\mathbf{F P}+\mathbf{P F}^{T}+\mathbf{Q}_{\text {spec }} \quad$ (or Eq. (3.26)) } \\
\hline \multirow{2}{*}{$\begin{array}{l}\text { Update } \\
t=t_{d}\end{array}$} & \multicolumn{3}{|c|}{$\begin{aligned} \hat{\mathbf{x}}^{+} & =\hat{\mathbf{x}}^{-}+\mathbf{K} \boldsymbol{\epsilon} \quad, \quad \mathbf{K}=\mathbf{P}^{-} \mathbf{H}^{T} \mathbf{W}^{-1} \\
\mathbf{P}^{+} & =[\mathbf{I}-\mathbf{K H}] \mathbf{P}^{-}[\mathbf{I}-\mathbf{K H}]^{T}+\mathbf{K} \mathbf{R} \mathbf{K}^{T}\end{aligned}$} \\
\hline & & $\begin{array}{l}:=\mathbf{z}-\mathbf{h}\left(\hat{\mathbf{x}}^{-}\right) \\
:=\mathbf{H P}^{-} \mathbf{H}^{T}+\mathbf{R}\end{array}$ & $\begin{aligned} \boldsymbol{\epsilon} & :=\mathbf{z}-\mathbf{h}\left(\hat{\mathbf{x}}^{-}\right)-\mathbf{b}_{h} \\
\mathbf{W} & :=\mathbf{H P}^{-} \mathbf{H}^{T}+\mathbf{R}+\mathbf{B}_{h}\end{aligned}$ \\
\hline Definitions & & $\mathbf{F}:=\left.\frac{\partial \mathbf{f}}{\partial \mathbf{x}}\right|_{\mathbf{x}=\hat{\mathbf{x}}(t)}$ & $\quad \mathbf{H}:=\left.\frac{\partial \mathbf{h}}{\partial \mathbf{x}}\right|_{\mathbf{x}=\hat{\mathbf{x}}\left(t_{d}^{-}\right)}$ \\
\hline
\end{tabular}

Table 3.2 summarizes the additional second-order terms required by the 
GSF. The terms are presented component-wise such that $\mathbf{b}_{f}=\left[b_{f_{i}}\right], \mathbf{b}_{h}=\left[b_{h_{k}}\right]$, and $\mathbf{B}_{h}=\left[B_{h_{j k}}\right]$. The notation $\partial^{2}(\cdot) / \partial \mathbf{x}^{2}$ is short for $\partial(\partial(\cdot) / \partial \mathbf{x})^{T} / \partial \mathbf{x}$.

Table 3.2: Second-Order Term Summary

\begin{tabular}{|l|c|}
\hline & GSF \\
\hline $\begin{array}{l}\text { Propagation } \\
t \in\left[t_{d-1}, t_{d}\right]\end{array}$ & $b_{f_{i}}=\frac{1}{2} \operatorname{trace}\left(\mathbf{F}_{i}^{\prime} \mathbf{P}\right)$ \\
\hline $\begin{array}{l}\text { Update } \\
t=t_{d}\end{array}$ & $b_{h_{k}}=\frac{1}{2} \operatorname{trace}\left(\mathbf{H}_{k}^{\prime} \mathbf{P}^{-}\right) \quad, \quad B_{h_{j k}}=\frac{1}{2} \operatorname{trace}\left(\mathbf{H}_{j}^{\prime} \mathbf{P}^{-} \mathbf{H}_{k}^{\prime} \mathbf{P}^{-}\right)$ \\
\hline Definitions & $\mathbf{F}_{i}^{\prime}:=\left.\frac{\partial^{2} f_{i}}{\partial \mathbf{x}^{2}}\right|_{\mathbf{x}=\hat{\mathbf{x}}(t)}, \quad \mathbf{H}_{k}^{\prime}:=\left.\frac{\partial^{2} h_{k}}{\partial \mathbf{x}^{2}}\right|_{\mathbf{x}=\hat{\mathbf{x}}\left(t_{d}^{-}\right)}$ \\
\hline
\end{tabular}

In the plots that follow, filter errors (or residuals) are displayed in blue, while the square root of the corresponding filter covariances are displayed in red. When appropriate, the square root of the corresponding covariance bounds or forecasts (Section 4.3) are displayed in green. 


\section{Chapter 4}

\section{Navigation Observability Analysis}

The observability analysis examines the relationship between various observability measures and the navigation performance of the EKF for several

different lunar mission cases. A summary of these mission cases can be found in Table 4.1. In a sense, observability measures the ability to estimate the state at time $t$ using measurements of the output of the system. More explicitly, observability is defined as follows:

Definition 4.0.1 (Observability). The state-space of a system is said to be observable if and only if, for any unknown initial state there exists a finite time such that knowledge of the input and the output over that finite time suffices to determine uniquely the initial state. [13]

In other words, a useful measure of observability should provide a sense of the ability to obtain an estimate of the state vector $\mathbf{x} \in \mathbb{R}^{n}$ given the time history of the measurement vector $\mathbf{z} \in \mathbb{R}^{m}$. Section 4.1 defines the observability measures used in this dissertation. 


\subsection{Observability Matrices and Measures}

Three measures of local observability are used in this dissertation: linear time-invariant, nonlinear, and linear time-varying. In the following sections the exact implications of the terms "observability" and "observable" should be inferred from the context of the observability measure that is being considered.

\subsubsection{Linear Time-Invariant}

The most common measure of observability is linear time-invariant (LTI) observability. Consider the following discrete deterministic, $n^{\text {th }}$-order linear system with time-invariant (i.e. constant) matrices $\boldsymbol{\Phi}$ and $\mathbf{H}$,

$$
\begin{aligned}
& \mathbf{x}\left(t_{d}\right)=\mathbf{\Phi} \mathbf{x}\left(t_{d-1}\right) \\
& \mathbf{z}\left(t_{d}\right)=\mathbf{H} \mathbf{x}\left(t_{d}\right)
\end{aligned}
$$

which has no inputs and $m$ noise-free outputs. Thus a series of $n$ measurements for $d=0,1, \ldots, n-1$ results in,

$$
\begin{aligned}
& \mathbf{z}\left(t_{1}\right)= \mathbf{H x}\left(t_{1}\right)=\mathbf{H x}\left(t_{1}\right) \\
& \mathbf{z}\left(t_{2}\right)=\mathbf{H x}\left(t_{2}\right)=\mathbf{H} \mathbf{\Phi} \mathbf{x}\left(t_{1}\right) \\
& \vdots \\
& \mathbf{z}\left(t_{n}\right)=\mathbf{H x}\left(t_{n}\right)=\mathbf{H} \boldsymbol{\Phi}^{n-1} \mathbf{x}\left(t_{1}\right)
\end{aligned}
$$

which in matrix form can then be written as,

$$
\boldsymbol{\zeta}=\boldsymbol{\Xi} \mathbf{x}\left(t_{1}\right)
$$

where $\boldsymbol{\zeta}$ and $\boldsymbol{\Xi}$ are defined as

$$
\boldsymbol{\zeta}:=\left[\begin{array}{c}
\mathbf{z}\left(t_{1}\right) \\
\mathbf{z}\left(t_{2}\right) \\
\vdots \\
\mathbf{z}\left(t_{n}\right)
\end{array}\right]_{n m \times 1} \quad \text { and } \quad \boldsymbol{\Xi}:=\left[\begin{array}{c}
\mathbf{H} \\
\mathbf{H} \boldsymbol{\Phi} \\
\vdots \\
\mathbf{H} \boldsymbol{\Phi}^{n-1}
\end{array}\right]_{n m \times n}
$$


Consequently, for $m=1$ in order for $\mathbf{x}\left(t_{1}\right)$ to be determined uniquely, the inverse of $\boldsymbol{\Xi}$ must exist. Although $m=1$ is a fairly limiting case, by considering the pseudo-inverse of the least-squares solution this concept can be expanded to not only incorporate instances for $m>1$, but also allow for noisy measurements. The unweighted (or equally weighted) least squares solution of Eq. (4.3) is as follows:

$$
\mathbf{x}\left(t_{1}\right)=\left(\boldsymbol{\Xi}^{T} \boldsymbol{\Xi}\right)^{-1} \boldsymbol{\Xi}^{T} \boldsymbol{\zeta}
$$

Notice that if $\boldsymbol{\Xi}$ is square, the pseudo-inverse reduces to the standard matrix inverse. If $\boldsymbol{\Xi}$ is not square however, the requirement for $\mathbf{x}\left(t_{1}\right)$ to be determined uniquely is now that the inverse of the Gram matrix $\boldsymbol{\Xi}^{T} \boldsymbol{\Xi}$ must exist. Since the existence of a matrix inverse is equivalent to the matrix being full rank, and since the rank of a Gram matrix is equivalent to the rank of the original matrix, then in either case the system of Eq. (4.1) is said to be observable by Definition 4.0.1 if, and only if, $\Xi$ is rank $n$ (full rank).

One drawback with the rank observability condition is its inherent polarity, the matrix is either rank $n$ or not rank $n$, that is, the system is either observable or not observable. Although when the rank is less than $n$ it still offers some integer measure of the number of linear combinations of the state that are observable, its inherent step-like nature does not allow for the visualization of the overall trend of the observability. Not only is there is no way to project the rank based on previous values, but also there is no means of observability comparison when the rank remains constant. Consequently the 
rank observability measure offers no continuous measure of the degree of the observability.

Alternatively, a necessary and sufficient condition for a square matrix to be full rank is that the determinant of the matrix is nonzero [25]. Thus, in the special case that $\boldsymbol{\Xi}$ is square $(m=1)$ the determinant is defined and can be used as an observability measure. In particular, the system is observable if, and only if, $\operatorname{det}(\boldsymbol{\Xi}) \neq 0$. While this again may seem like a polar or binary observability condition, the continuous nature of the determinant can show if the determinant is approaching zero before the singularity actually occurs. Furthermore, recalling that the rank of a Gram matrix is equivalent to the rank of the original matrix, it is not surprising that a non-square matrix is full rank, if, and only if, the determinant of its Gram matrix is a nonzero [13]. Thus, the natural extension of the determinant observability condition is that $\operatorname{det}\left(\boldsymbol{\Xi}^{T} \boldsymbol{\Xi}\right) \neq 0$. However, since when $\boldsymbol{\Xi}$ is square $\operatorname{det}\left(\boldsymbol{\Xi}^{T} \boldsymbol{\Xi}\right)=\operatorname{det}(\boldsymbol{\Xi})^{2}$ [25], for consistency between the $m=1$ determinant and the $m>1$ "pseudodeterminant," the actual LTI observability measure computed is $\operatorname{det}\left(\boldsymbol{\Xi}^{T} \boldsymbol{\Xi}\right)^{1 / 2}$. Expanding this concept to the $n^{\text {th }}$-order continuous LTI system with vector outputs (no inputs),

$$
\begin{aligned}
& \dot{\mathbf{x}}=\mathbf{F x} \\
& \mathbf{z}=\mathbf{H} \mathbf{x}
\end{aligned}
$$

results in what is commonly referred to as the LTI observability matrix $\mathcal{O}$ [19],

$$
\mathcal{O}:=\left[\begin{array}{c}
\mathbf{H} \\
\mathbf{H F} \\
\vdots \\
\mathbf{H F}^{n-1}
\end{array}\right]_{n m \times n}
$$


where again $n$ is the length of the state vector $\mathbf{x}$ and $m$ is the length of the measurement vector $\mathbf{z}$. This similarly requires that the matrix $\mathcal{O}$ be rank $n$, which is also equivalent to $\mathcal{O}$ having $n$ linearly independent rows [25]. Thus, considering the previous discussion on the benefit of using the determinant observability measure, results in the LTI observability condition:

Definition 4.1.1 (LTI Observability Condition). The continuous $n^{\text {th }}$ order, linear time-invariant system of Eq. (4.6) is said to be locally observable if, and only if, $\operatorname{det}\left(\boldsymbol{\mathcal { O }}^{T} \boldsymbol{\mathcal { O }}\right)^{1 / 2} \neq 0$, where the matrix $\mathcal{O}$ is defined in Eq. (4.7).

Although the systems examined in this dissertation are neither linear nor time-invariant, by defining $\mathbf{F}$ and $\mathbf{H}$ as the evaluation of Eqs. (3.14) and (3.21) on some nominal or reference trajectory at a "frozen" instant in time, examination of the LTI observability condition can still provide useful insight into the local observability of the systems at that time. However, since in doing so the time-invariant observability measure is examined at essentially independent instances in time, it should be noted that the measure will not account for any observability gained from a build-up of measurement information. Thus the time-invariant analysis is typically most applicable during early portions of the navigation. Also, it is known that even if the system is observable at "frozen" points in time, it may not be observable. 


\subsubsection{Nonlinear}

The concept of observability has also been extended to certain classes of continuous, $n^{\text {th }}$-order nonlinear systems of the form,

$$
\begin{aligned}
& \dot{\mathbf{x}}=\mathbf{f}(\mathbf{x}) \\
& \mathbf{z}=\mathbf{h}(\mathbf{x})
\end{aligned}
$$

which, aside from the noise-free assumptions and measurement discretization, is equivalent to the formulation of the systems presented in Section 3.1. Consider for the moment that it is desirable in Eq. (4.8) to find a coordinate transformation in some neighborhood of an initial $\mathbf{x}$, such that the resulting error dynamics are linear. Isidori [29] calls this problem the Observer Linearization Problem and use the LTI rank observability condition of the transformed system to prove that the problem is solvable only if the so-called nonlinear observability matrix, $\mathcal{N}$, is rank $n$. The matrix $\mathcal{N}$ is composed of the Lie derivatives of the system in Eq. (4.8), such that

$$
\mathcal{N}:=\left[\begin{array}{c}
\mathcal{N}_{1} \\
\mathcal{N}_{2} \\
\vdots \\
\mathcal{N}_{n}
\end{array}\right]_{n m \times n}=\frac{\partial}{\partial \mathbf{x}}\left[\begin{array}{c}
\mathbf{h} \\
\mathcal{N}_{1} \mathbf{f} \\
\vdots \\
\mathcal{N}_{n-1} \mathbf{f}
\end{array}\right] \quad, \quad \begin{aligned}
& \mathcal{N}_{1}=\mathbf{H} \\
& \mathcal{N}_{i}=\frac{\partial}{\partial \mathbf{x}}\left(\mathcal{N}_{i-1} \mathbf{f}\right)
\end{aligned}
$$

for $i=2, \ldots, n$ and where again $n$ is the dimension of the state vector $\mathbf{x}$ and $m$ is the dimension of the measurement vector $\mathbf{z}$.

The concept of the Observer Linearization Problem and the resulting observability condition are relevant for the systems of this dissertation, especially considering that this is essentially what is already done in the linearized and extended Kalman filters. Recall from Section 3.2 that the resulting error 
dynamics are linear such that $\dot{\mathbf{e}} \approx \mathbf{F e}$ (for the noise free assumption). Additional validity is given to this (first-order) linearization since, as will be shown in Section 5.3, the second-order terms are negligible.

Due to the limitations of the rank observability condition discussed in Section 4.1.1, the determinant observability condition is again applied and Isidori's condition results in the definition:

Definition 4.1.2 (Nonlinear Observability Condition). The continuous $n^{\text {th }}$-order, nonlinear system of Eq. (4.8), is said to be locally observable only if $\operatorname{det}\left(\mathcal{N}^{T} \mathcal{N}\right)^{1 / 2} \neq 0$, where the matrix $\mathcal{N}$ is defined in Eq. (4.9).

As with the LTI observability measure, since the systems being examined instead employ discrete measurements, the nonlinear observability measure is considered at "frozen" instances in time. The analogous formulation of the two measures allows for a direct comparison of the effects of the nonlinearities. The limitation of the nonlinear observability measure is the inherent requirement of the computation of $n$ partial derivatives. Consequently, for most applications nonlinear observability analysis is impractical or requires simplifying assumptions to reduce the state-space to a more manageable size.

\subsubsection{Linear Time-Varying}

As discussed in the previous sections, the prominent shortcoming in the time-invariant analysis is the inability to account for a build-up in observability due to accumulated measurement information. Recall that the reason for this 
was that in order to treat $\mathbf{F}$ and $\mathbf{H}$ as roughly time-invariant the matrices needed to be examined at separate "frozen" instances in time. Consequently, reconsidering Eq. (4.1) with time-varying $\boldsymbol{\Phi}=\boldsymbol{\Phi}\left(t_{d}, t_{d-1}\right)$ and $\mathbf{H}=\mathbf{H}\left(t_{d}\right)$, a series of $d$ measurements now results in,

$$
\begin{aligned}
& \mathbf{z}\left(t_{1}\right)=\mathbf{H}\left(t_{1}\right) \mathbf{x}\left(t_{1}\right)=\mathbf{H}\left(t_{1}\right) \mathbf{x}\left(t_{1}\right) \\
& \mathbf{z}\left(t_{2}\right)=\mathbf{H}\left(t_{2}\right) \mathbf{x}\left(t_{2}\right)=\mathbf{H}\left(t_{2}\right) \boldsymbol{\Phi}\left(t_{2}, t_{1}\right) \mathbf{x}\left(t_{1}\right) \\
& \vdots \\
& \mathbf{z}\left(t_{d}\right)=\mathbf{H}\left(t_{d}\right) \mathbf{x}\left(t_{d}\right)=\mathbf{H}\left(t_{d}\right) \boldsymbol{\Phi}\left(t_{d}, t_{1}\right) \mathbf{x}\left(t_{1}\right)
\end{aligned}
$$

which in matrix form can again be written as,

$$
\boldsymbol{\zeta}\left(t_{d}\right)=\boldsymbol{\Xi}\left(t_{d}\right) \mathbf{x}\left(t_{1}\right)
$$

except $\boldsymbol{\zeta}$ and $\boldsymbol{\Xi}$ are now defined as

$$
\boldsymbol{\zeta}\left(t_{d}\right):=\left[\begin{array}{c}
\mathbf{z}\left(t_{1}\right) \\
\mathbf{z}\left(t_{2}\right) \\
\vdots \\
\mathbf{z}\left(t_{d}\right)
\end{array}\right]_{d m \times 1} \quad \text { and } \quad \boldsymbol{\Xi}\left(t_{d}\right):=\left[\begin{array}{c}
\mathbf{H}\left(t_{1}\right) \\
\mathbf{H}\left(t_{2}\right) \boldsymbol{\Phi}\left(t_{2}, t_{1}\right) \\
\vdots \\
\mathbf{H}\left(t_{d}\right) \mathbf{\Phi}\left(t_{d}, t_{1}\right)
\end{array}\right]_{d m \times n}
$$

Notice that since by definition,

$$
\boldsymbol{\Phi}\left(t_{d}, t_{1}\right)=\prod_{i=d}^{2} \boldsymbol{\Phi}\left(t_{i}, t_{i-1}\right)
$$

a time-invariant $\boldsymbol{\Phi}\left(t_{i}, t_{i-1}\right)=\boldsymbol{\Phi}$ results in $\boldsymbol{\Phi}\left(t_{d}, t_{1}\right)=\boldsymbol{\Phi}^{d-1}$. Thus, for a timeinvariant $\mathbf{H}\left(t_{d}\right)=\mathbf{H}$ with $d=n$, Eq. (4.12) reduces exactly to Eq. (4.4).

As in Section 4.1.1, the time-varying version of the system of Eq. (4.1) is said to be observable by Definition 4.0 .1 if, and only if, $\boldsymbol{\Xi}^{T}\left(t_{d-1}\right) \boldsymbol{\Xi}\left(t_{d-1}\right)$ is rank $n$. For a discrete-time, time-varying system this Gram matrix is appropriately 
named the observability Gramian and is more commonly written in the form,

$$
\mathcal{M}\left(t_{d}\right):=\boldsymbol{\Xi}^{T}\left(t_{d}\right) \boldsymbol{\Xi}\left(t_{d}\right)=\sum_{k=1}^{d} \boldsymbol{\Phi}^{T}\left(t_{k}, t_{1}\right) \mathbf{H}^{T}\left(t_{k}\right) \mathbf{H}\left(t_{k}\right) \boldsymbol{\Phi}\left(t_{k}, t_{1}\right)
$$

or equivalently in recursive form,

$$
\mathcal{M}\left(t_{d}\right)=\mathcal{M}\left(t_{d-1}\right)+\boldsymbol{\Phi}^{T}\left(t_{d}, t_{1}\right) \mathbf{H}^{T}\left(t_{d}\right) \mathbf{H}\left(t_{d}\right) \boldsymbol{\Phi}\left(t_{d}, t_{1}\right)
$$

with the initial condition that $\mathcal{M}\left(t_{0}\right)=\mathbf{0}$, since no measurements are taken at time $t_{0}$. With the observability Gramian defined, the linear time-varying observability condition results in the definition:

Definition 4.1.3 (Observability Gramian Condition). The discrete $n^{t h}$ order, linear time-varying version of the system of Eq. (4.1) is said to be locally observable if, and only if, the matrix $\mathcal{M}\left(t_{d}\right)$, defined in Eq. (4.14), is rank $n$.

Notice in Eq. (4.14) that since the rank of each term in the summation is at most $m$, there is some minimal number $d \geq n / m$ of measurements that must be processed before $\mathcal{M}\left(t_{d}\right)$ is rank $n$ [39]. Therefore the number of measurements required to satisfy Definition 4.1 .3 could also be used as a measure of observability. However once satisfied, since each term in the summation is positive semidefinite, $\mathcal{M}\left(t_{d}\right)$ will remain full rank and the determinant will continue to grow more positive. Consequently, these measures offer little insight once the system becomes observable. This is of particular concern for the missions examined in this dissertation, where since $n / 2 \leq m<n$, typically only two measurements are required for $\mathcal{M}\left(t_{d}\right)$ to become rank $n$. 
However, although satisfying Definition 4.1.3 exhausts the benefits of the standard observability measures, it automatically implies that the observability Gramian has become invertible, which in turn introduces an entirely new set of observability measures. One example of this is a measure known as the dilution of precision (DOP). Section 4.3.3 expands upon the DOP metric to provide a rough observability-based forecast of the state error covariance.

\subsection{Observer Geometry Significance}

By examining the LTI observability condition associated with the observer locations and formation geometry, it is possible to develop guidelines that not only predict the expected navigation performance of a particular geometry, but also which geometries should be avoided entirely. To this end, using Definition 4.1.1 three mission cases were examined: Cases 0, 1, and 2 in Table 4.1.

\begin{tabular}{cccccc}
\multicolumn{7}{c}{ Table 4.1: Lunar Mission Cases } \\
\hline \hline Case & Spacecraft & $n$ & Measurement Profile and Frequency & $m$ \\
\hline 0 & 1 & 4 & inertial only & every hour & 2 \\
1.0 & 2 & 12 & inertial only & every minute & 6 \\
1.1 & 2 & 12 & relative fusion & every minute & 7 \\
2.0 & 6 & 36 & inertial only & every minute & 18 \\
2.1 & 6 & 36 & relative fusion & every minute & 33 \\
\hline \hline
\end{tabular}

As outlined in Section 2.3.1, Case 0 examines a cluster of spacecraft on a cislunar transfer trajectory, while Cases 1 and 2 examine two formation variation in a circular polar orbit about the Moon. Cases x.0 and x.1 are identical 
(including noise values) with the sole exception that the x.0 cases use only inertial measurements, while the x.1 cases fuse inertial and relative measurements. All three cases are initialized using a moderate level of initial covariance as described in Table 5.1. Additional details regarding the observer locations, formation geometries, dynamics, measurement profiles, and filter models can be found Sections 2.3.1 and 2.3.2. Finally, in Sections 4.2.1 and 4.2.2, unless otherwise noted, the terms "observability" and "observable" always refer to observability in the LTI sense.

\subsubsection{Cluster-Observer Geometry}

In the cislunar transfer mission segment, Case 0, a single cluster of spacecraft is tracked in two-dimensional space $(n=4)$ using two tracking stations $(m=2)$ located at each of the two equilateral libration points $\left(L_{4}\right.$ and $\left.L_{5}\right)$. Recalling Eqs. (2.20) and (2.26),

$$
\mathbf{F}=\left[\begin{array}{c|c}
\mathbf{0} & \mathbf{I} \\
\hline \mathbf{G} & \mathbf{0}
\end{array}\right] \quad, \quad \mathbf{H}=\left[\begin{array}{c|c}
\boldsymbol{\rho}_{1}^{T} / \rho_{1} & \mathbf{0}^{T} \\
\boldsymbol{\rho}_{2}^{T} / \rho_{2} & \mathbf{0}^{T}
\end{array}\right]
$$

which results in the following LTI observability matrix, $\mathcal{O}$ :

$$
\boldsymbol{O}=\left[\begin{array}{cc}
\boldsymbol{\rho}_{1}^{T} / \rho_{1} & \mathbf{0}^{T} \\
\boldsymbol{\rho}_{2}^{T} / \rho_{2} & \mathbf{0}^{T} \\
\mathbf{0}^{T} & \boldsymbol{\rho}_{1}{ }^{T} / \rho_{1} \\
\mathbf{0}^{T} & \boldsymbol{\rho}_{2}{ }^{T} / \rho_{2} \\
\boldsymbol{\rho}_{1}^{T} \mathbf{G} / \rho_{1} & \mathbf{0}^{T} \\
\boldsymbol{\rho}_{2}^{T} \mathbf{G} / \rho_{2} & \mathbf{0}^{T} \\
\mathbf{0}^{T} & \boldsymbol{\rho}_{1}{ }^{T} \mathbf{G} / \rho_{1} \\
\mathbf{0}^{T} & \boldsymbol{\rho}_{2}{ }^{T} \mathbf{G} / \rho_{2}
\end{array}\right]
$$

Since the cluster is treated as one spacecraft, the $j$ subscript is dropped for convenience. 
Similarly, since the dimension of the state-space is manageable $(n=4)$, the nonlinear observability matrix is also considered here. For $m$ inertial range measurements, the nonlinear observability matrix $\mathcal{N}$ can be written in terms of the LTI observability matrix $\mathcal{O}$, such that,

$$
\mathcal{N}=\mathcal{O}+\mathcal{E}
$$

where, for $m=2, \mathcal{O}$ is define by Eq. (4.17) and $\mathcal{E}$ has the formulation:

$$
\mathcal{E}=\left[\begin{array}{cc}
\mathcal{E}_{1} & 0 \mathcal{E}_{0} \\
\mathcal{E}_{2} & 1 \mathcal{E}_{1} \\
\mathcal{E}_{3} & 2 \mathcal{E}_{2} \\
\mathcal{E}_{4} & 3 \mathcal{E}_{3}
\end{array}\right] \quad \text { where } \quad \mathcal{E}_{i}=\left[\begin{array}{c}
\mathcal{E}_{i 1} \\
\vdots \\
\mathcal{E}_{i m}
\end{array}\right]
$$

with $\mathcal{E}_{0}=\mathcal{E}_{1}=\mathbf{0}$. The submatrices $\mathcal{E}_{i k}$, for $i=2,3,4$ and the $k^{\text {th }}$ measurement $(k=1, \ldots, m)$, can then be written as,

$$
\begin{aligned}
\mathcal{E}_{2 k}= & \frac{1}{\rho_{k}^{3}}\left[-\left(\boldsymbol{\rho}_{k}^{T} \mathbf{v}\right) \boldsymbol{\rho}_{k}^{T}+\rho_{k}^{2} \mathbf{v}^{T}\right] \\
\mathcal{E}_{3 k}= & \frac{1}{\rho_{k}^{3}}\left[\psi_{k} \boldsymbol{\rho}_{k}^{T}-2\left(\boldsymbol{\rho}_{k}^{T} \mathbf{v}\right) \mathbf{v}^{T}+\rho_{k}^{2} \mathbf{g}^{T}\right] \\
\mathcal{E}_{4 k}= & \frac{1}{\rho_{k}^{3}}\left[\eta_{k} \boldsymbol{\rho}_{k}^{T}+3 \psi_{k} \mathbf{v}^{T}-3\left(\boldsymbol{\rho}_{k}^{T} \mathbf{v}\right) \mathbf{g}^{T}\right. \\
& \left.+\left(-3\left(\boldsymbol{\rho}_{k}^{T} \mathbf{v}\right) \boldsymbol{\rho}_{k}^{T}+4 \rho_{k}^{2} \mathbf{v}^{T}\right) \mathbf{G}+\rho_{k}^{2} \mathbf{v}^{T} \mathbf{C}_{k}\right]
\end{aligned}
$$

where the scalars $\psi_{k}$ and $\eta_{k}$ are defined as

$$
\begin{aligned}
& \psi_{k}:=3 \frac{\left(\boldsymbol{\rho}_{k}^{T} \mathbf{v}\right)^{2}}{\rho_{k}^{2}}-\boldsymbol{\rho}_{k}^{T} \mathbf{g}-\mathbf{v}^{T} \mathbf{v} \\
& \eta_{k}:=12 \frac{\left(\boldsymbol{\rho}_{k}^{T} \mathbf{v}\right)^{3}}{\rho_{k}^{4}}-9 \psi_{k} \frac{\boldsymbol{\rho}_{k}^{T} \mathbf{v}}{\rho_{k}^{2}}-3 \mathbf{v}^{T} \mathbf{g}-\boldsymbol{\rho}_{k}^{T} \mathbf{G} \mathbf{v}
\end{aligned}
$$

Finally, the matrix $\mathbf{C}_{k}$ in $\boldsymbol{E}_{4 k}$ is defined such that,

$$
\frac{\partial\left(\mathbf{G} \boldsymbol{\rho}_{k}\right)}{\partial \mathbf{r}}=\mathbf{G}+\mathbf{C}_{k}
$$


which results in

$$
\mathbf{C}_{k}:=\frac{3 \mu}{\|\mathbf{r}\|^{5}}\left(\mathbf{r} \boldsymbol{\rho}_{k}^{T}+\boldsymbol{\rho}_{k} \mathbf{r}^{T}-5 \frac{\left(\boldsymbol{\rho}_{k}^{T} \mathbf{r}\right)}{\|\mathbf{r}\|^{2}} \mathbf{r r}^{T}+\left(\boldsymbol{\rho}_{k}^{T} \mathbf{r}\right) \mathbf{I}\right)
$$

Thus, Eq. (4.18) offers a direct and convenient comparison between the LTI and nonlinear observability matrices.

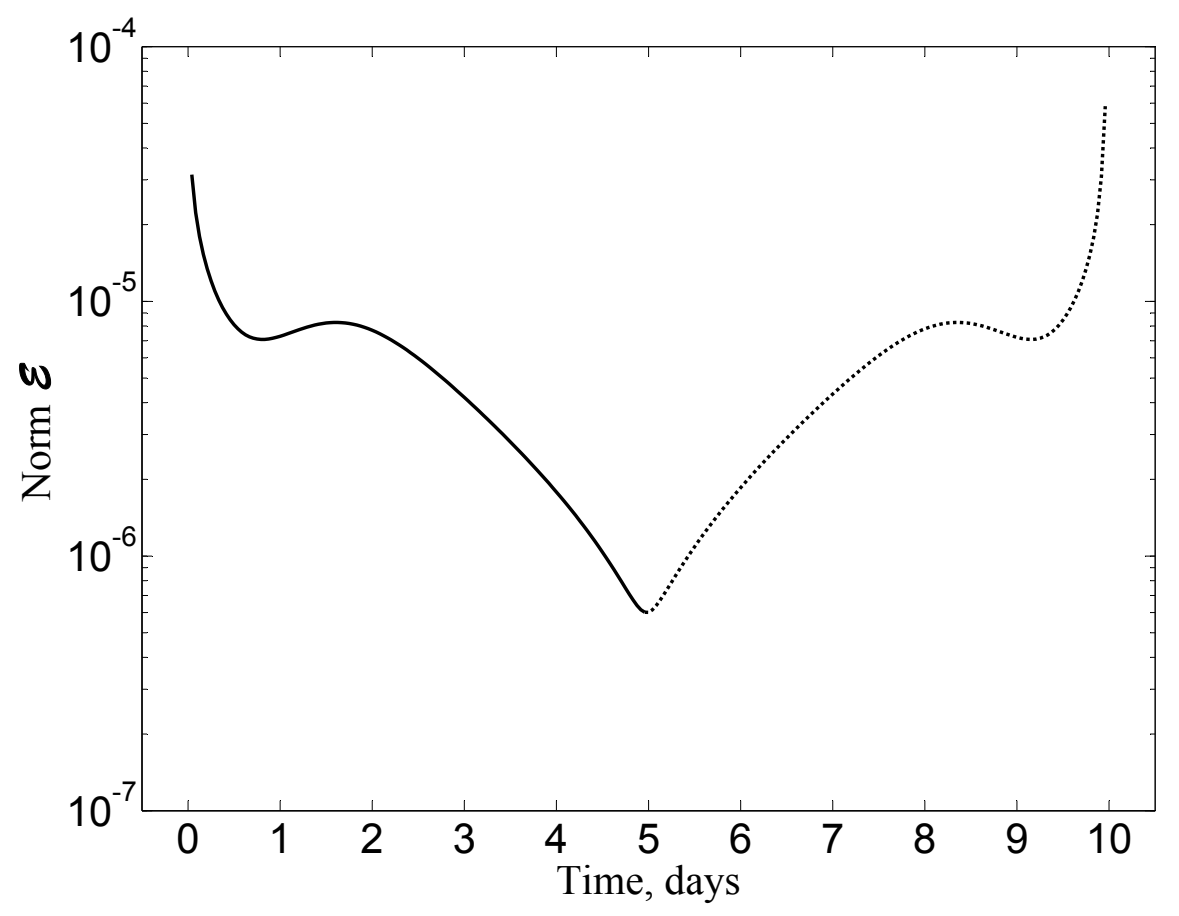

Figure 4.1: Time history of $\|\mathcal{E}\|$.

In particular, if $\mathcal{E} \approx \mathbf{0}$, then the nonlinearities are negligible within the context of Section 4.1.2. It turns out, as seen in Figure 4.1, it is in fact the case that $\mathcal{E} \approx 0$. While the reduction seen near 5 days in Figure 4.1 is the 
consequence of the magnitude of the gravity terms, which are small far from the Earth, the overall small magnitude of $\mathcal{E}$ can be explained in terms of the range measurement magnitude. Notice in Eq. (4.20) that each $\mathcal{E}_{i k}$ is roughly dominated by at least a $1 / \rho_{k}$ term. Thus, as will be proven in Section 5.3 , the significance of the nonlinearities is inversely proportional to the magnitude of the range measurement. Consequently, with range measurements on the order of $10^{5} \mathrm{~km}$, these terms become negligible and the nonlinear observability matrix is essentially equivalent to the LTI observability matrix. In fact, since the observability measures $\operatorname{det}\left(\mathcal{O}^{T} \mathcal{O}\right)^{1 / 2}$ and $\operatorname{det}\left(\mathcal{N}^{T} \mathcal{N}\right)^{1 / 2}$ agree to an order of $10^{-9}$ or better, the nonlinear observability is no longer considered here.

Returning to Eq. (4.17), at this point it is useful to consider the magnitude of the matrix $\mathbf{G}$. In particular, since $\mathbf{G}$ is symmetric, its 2-norm is the maximum of the absolute value of its eigenvalues. Thus, analytically computing the eigenvalues of $\mathbf{G}$ results in

$$
\boldsymbol{\lambda}(\mathbf{G})=\frac{\mu}{\|\mathbf{r}\|^{3}}\left[\begin{array}{c}
-1 \\
-1 \\
2
\end{array}\right] \quad \text { and } \quad\|\mathbf{G}\|=\max |\boldsymbol{\lambda}(\mathbf{G})|=\frac{2 \mu}{\|\mathbf{r}\|^{3}}
$$

where here $\mu$ is the gravitational constant of Earth. Therefore, since $\|\mathbf{r}\|>R_{\oplus}$, at most $\|\mathbf{G}\|<3.07 \times 10^{-6} 1 / \mathrm{s}^{2}$ and when $\|\mathbf{r}\|=a_{\oplus / \mathcal{S}}$ at the end of the transfer, $\|\mathbf{G}\|=1.40 \times 10^{-11} 1 / \mathrm{s}^{2}$. Consequently, for simplification these terms are considered negligible when examining the observability matrix analytically. This implies that $\mathcal{O}$ approximately only contains the rows resulting from $\mathbf{H}$ and HF. However, this simplification is not made when computing the observability measure $\operatorname{det}\left(\mathcal{O}^{T} \mathcal{O}\right)^{1 / 2}$ numerically. 


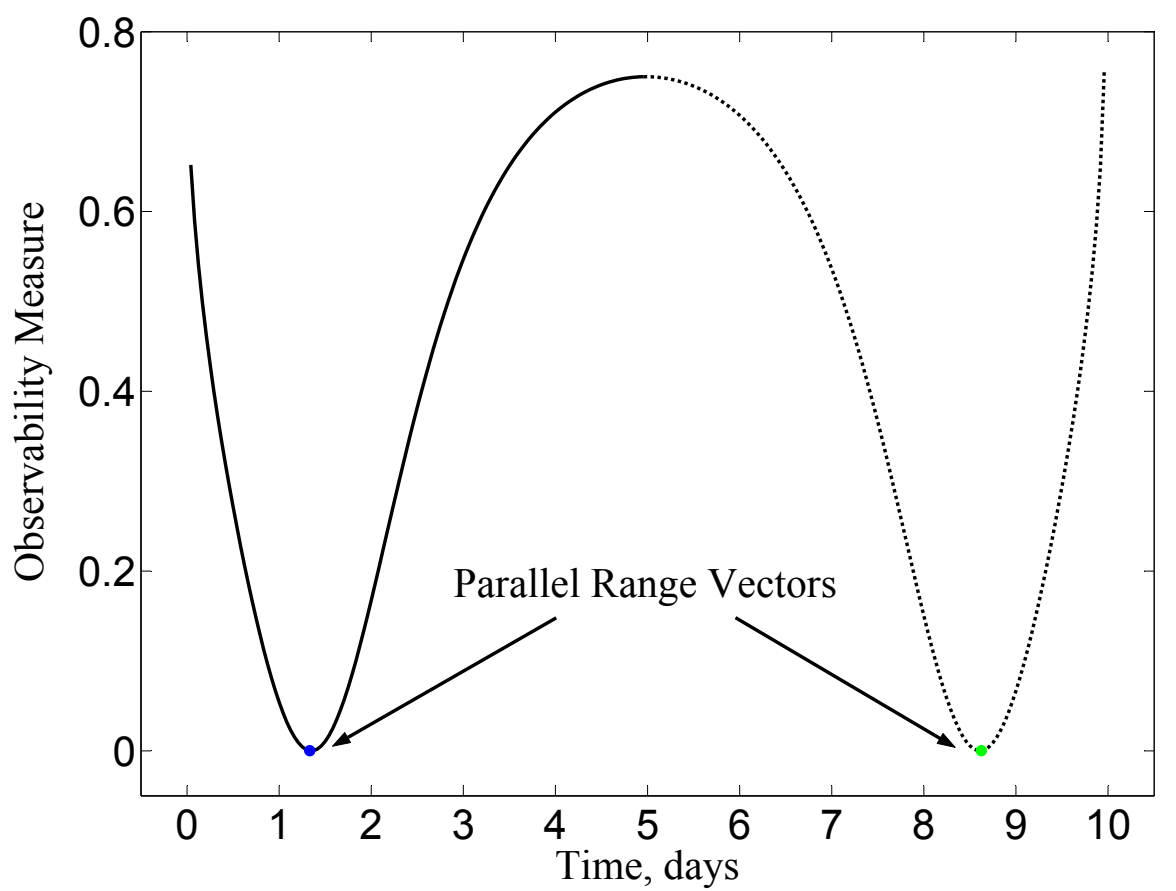

Figure 4.2: Numerical detection for Case 0.

Since $n=4$, the remaining four rows of $\mathcal{O}$ must all be linearly independent in order to satisfy the observability condition in Definition 4.1.1. Examining $\boldsymbol{O}$ leads to the conclusion that if $\boldsymbol{\rho}_{1}$ and $\boldsymbol{\rho}_{2}$ are parallel, the number of linearly independent rows is reduced to two and thus the observability condition is violated.

This can be illustrated both numerically and geometrically. Numerically this can be detected by computing the observability measure, as seen in Figure 4.2. Recall that the observability condition is violated when the observ- 


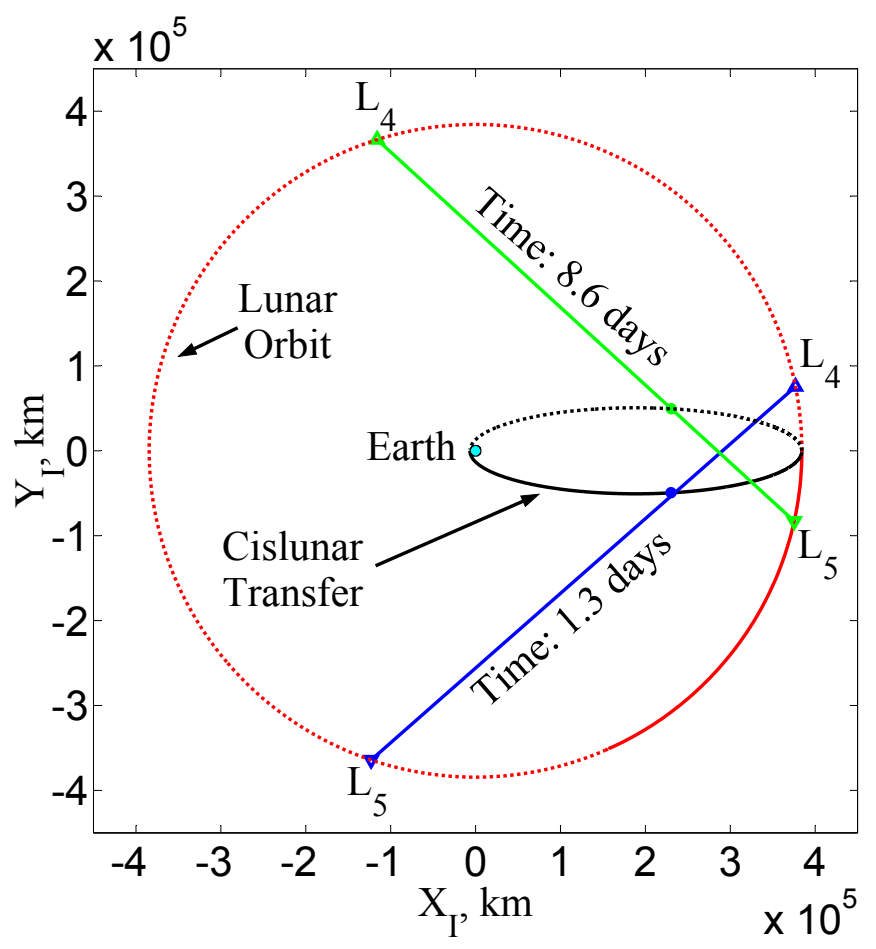

Figure 4.3: Geometrical detection for Case 0.

ability measure $\operatorname{det}\left(\mathcal{O}^{T} \mathcal{O}\right)^{1 / 2}=0$. Geometrically, the observability violation occurs when the spacecraft crosses a line connecting the two tracking stations. Figure 4.3 shows the cislunar transfer trajectory (black) with the Earth (cyan) at the origin, the trajectory of the Moon (red), and libration points $L_{4}$ (triangle) and $L_{5}$ (upsidedown triangle) at two discrete times (blue and green). The dotted lines denote parts of the complete trajectories not traversed during the transfer.

In both Figures 4.2 and 4.3 the blue and green markings denote events 

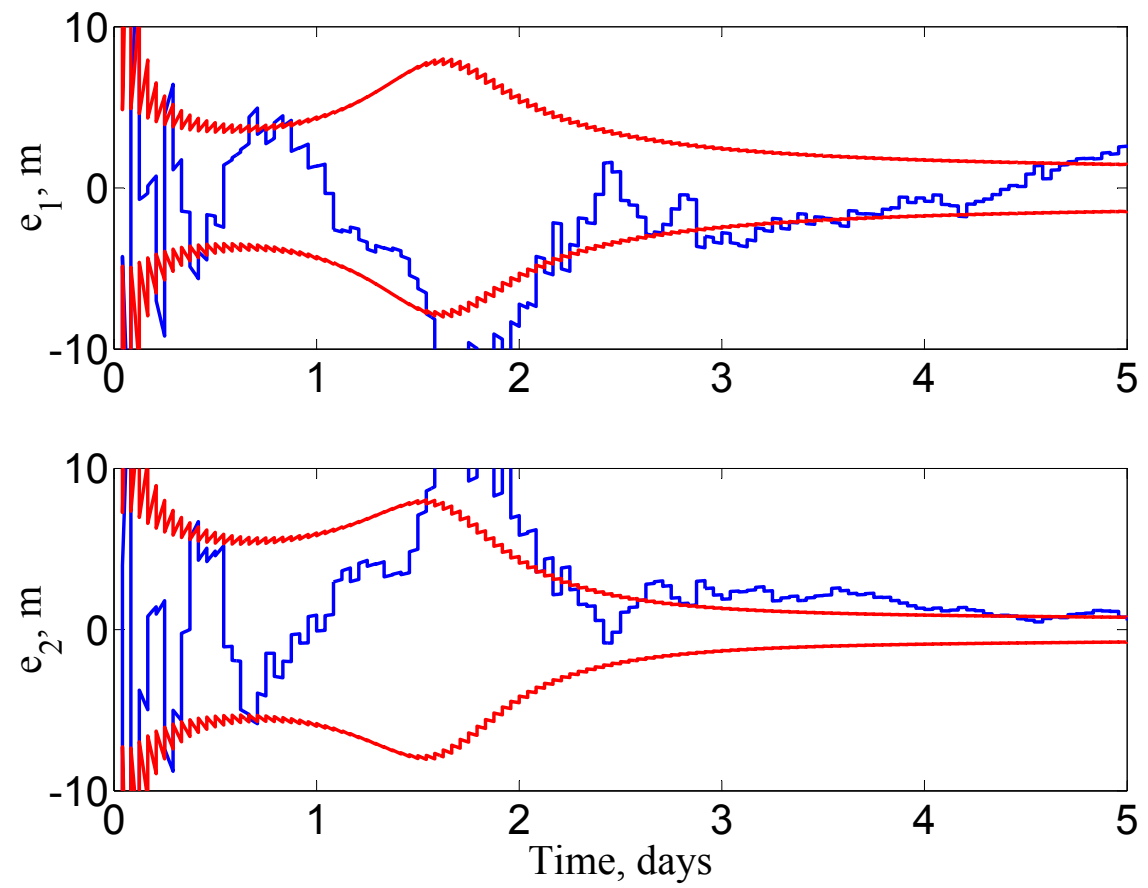

Figure 4.4: Time history of the inertial position errors for Case 0.

occurring at the same respective times. In other words, the times where the blue and green points occur in Figure 4.2 have also been marked on the black trajectory in Figure 4.3. At these times a line connects the $L_{4}$ tracking station and the $L_{5}$ tracking station, indicating that both range vectors are parallel.

The corresponding navigation state error can be seen in Figures 4.4 and 4.5. Notice that the bulges in covariance occurring around 1.5 days coincide with the violation of the observability condition, as seen in Figure 4.2. Had the gravitational effects of the Moon been included, another slight bulge 

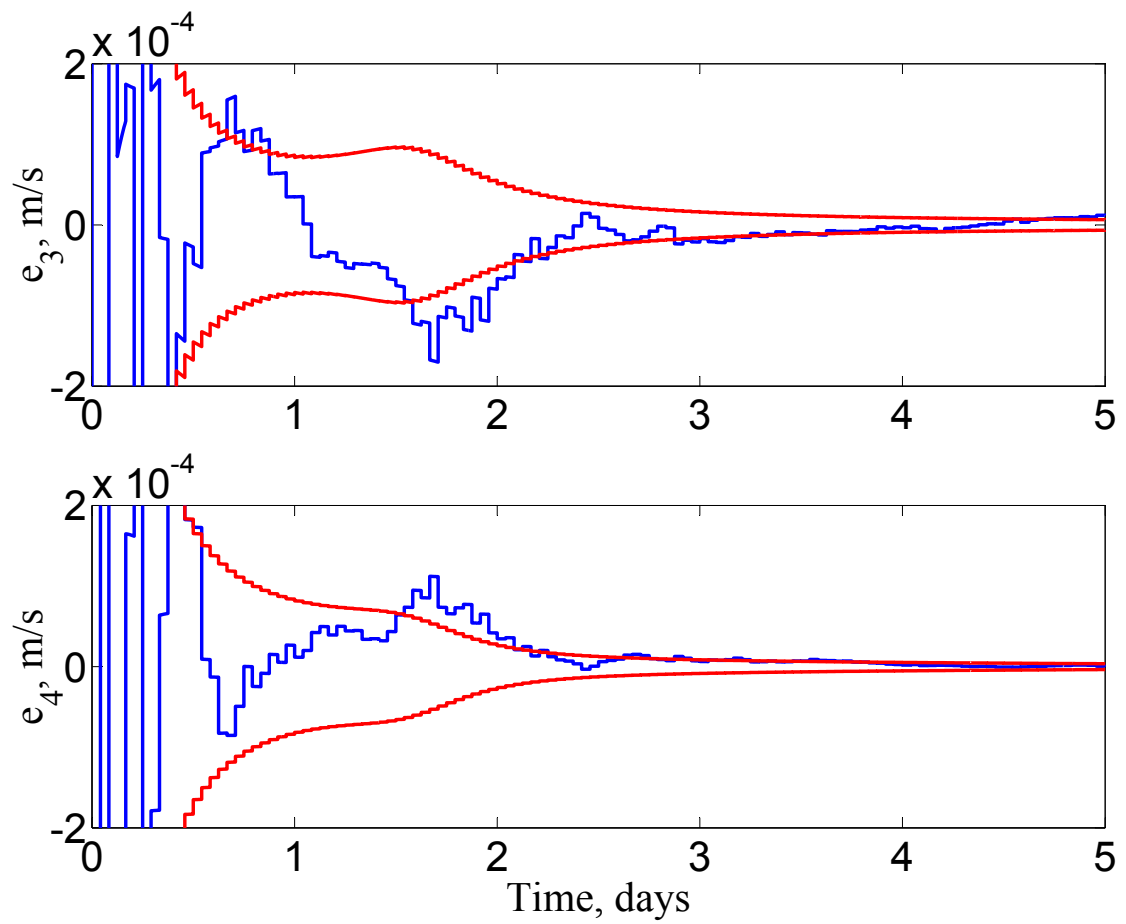

Figure 4.5: Time history of the inertial velocity errors for Case 0.

would have occurred during the lunar approach. However, this bulge would not have been due to a loss of observability in the LTI sense, but instead due to the rapid changes in velocity during the lunar flyby. In fact, as discussed in [4], planetary approach may actually enhance nonlinear local observability. Regardless, once through the flyby (or after orbital insertion) the state estimation error covariances quickly begin to converge again.

As seen in Figure 4.6, the residuals are approximately zero-mean, white noise with a standard deviation of about $5 \mathrm{~m}$, which as discussed in Section 

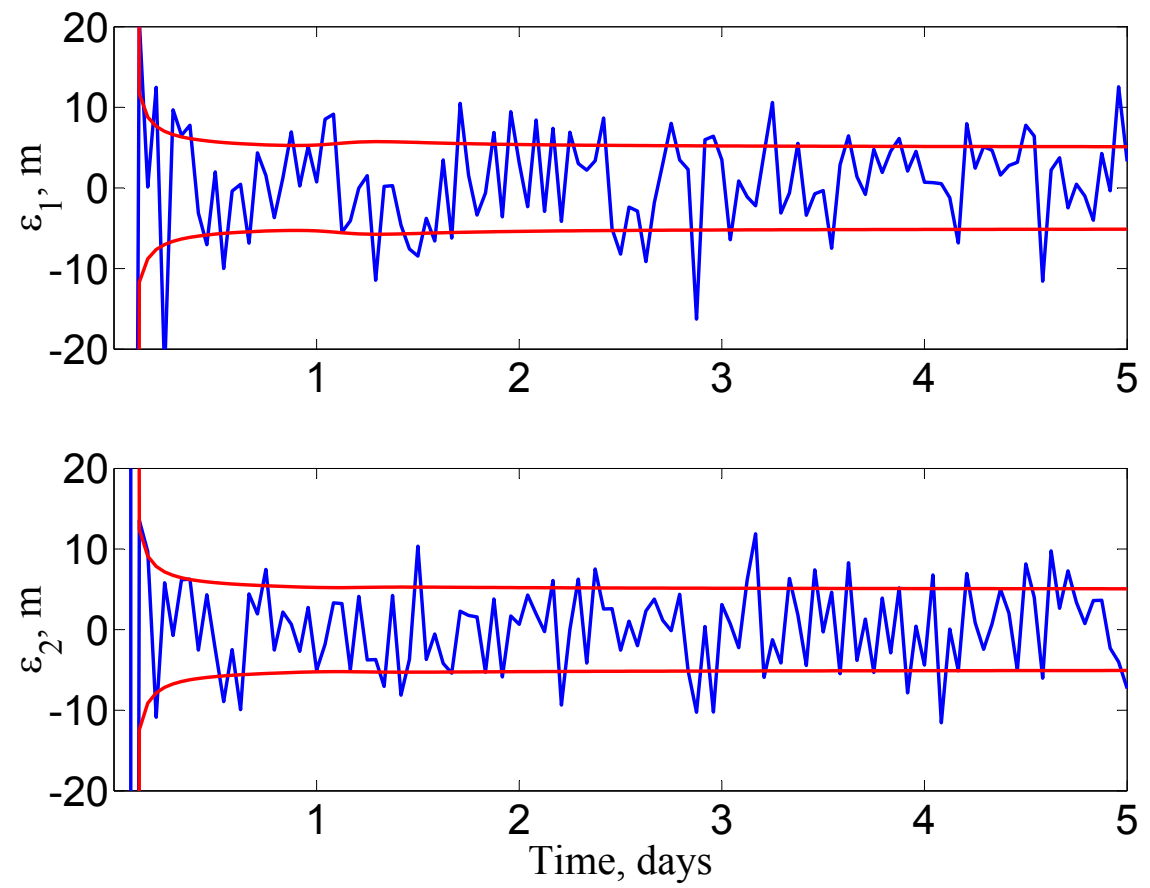

Figure 4.6: Time history of the range residuals for Case 0.

\section{4 , indicates the filter is working properly.}

Via simulation, it has been shown that examination of the observability condition can help detect potential problems in the navigation performance. In particular, for this scenario, the two-dimensional system becomes unobservable when the range vectors are parallel. The next two mission cases extend this concept to three-dimensional space while also examining the advantages and complexities of adding additional spacecraft. 


\subsubsection{Formation-Observer Geometry}

In the circular polar orbit mission segment, Cases 1 and 2, the cluster separates and a formation of spacecraft is tracked in three-dimensions using three tracking stations, with two located at each of the equilateral libration points $\left(L_{4}\right.$ and $\left.L_{5}\right)$ and one at the co-linear libration point on the far-side of the Moon $\left(L_{2}\right)$. As seen in Table 4.1, each mission examines two measurement scenarios: without (x.0) and with (x.1) the use of relative range measurements. For all cases, the dynamics matrix $\mathbf{F}$ is defined using Eq. (2.20).

Applying Eq. (2.26), the mapping matrix $\mathbf{H}$ for Case 1.1 is given by

$$
\mathbf{H}=\left[\begin{array}{cc|cc}
\boldsymbol{\rho}_{11}^{T} / \rho_{11} & \mathbf{0}^{T} & \mathbf{0}^{T} & \mathbf{0}^{T} \\
\boldsymbol{\rho}_{21}^{T} / \rho_{21} & \mathbf{0}^{T} & \mathbf{0}^{T} & \mathbf{0}^{T} \\
\boldsymbol{\rho}_{31}^{T} / \rho_{31} & \mathbf{0}^{T} & \mathbf{0}^{T} & \mathbf{0}^{T} \\
\mathbf{0}^{T} & \boldsymbol{\rho}_{12}^{T} / \rho_{12} & \mathbf{0}^{T} & \mathbf{0}^{T} \\
\mathbf{0}^{T} & \boldsymbol{\rho}_{22}^{T} / \rho_{22} & \mathbf{0}^{T} & \mathbf{0}^{T} \\
\mathbf{0}^{T} & \boldsymbol{\rho}_{32}^{T} / \rho_{32} & \mathbf{0}^{T} & \mathbf{0}^{T} \\
\hline \hline-\boldsymbol{\rho}_{12}^{T} / \rho_{12} & \boldsymbol{\rho}_{12}^{T} / \rho_{12} & \mathbf{0}^{T} & \mathbf{0}^{T}
\end{array}\right]
$$

where the additional partition (below the double line) is used to separate the inertial and relative measurement rows. While the full observability matrix is too large to present here, the terms of Eq. (4.7) can be found for $i=$ $0,1, \ldots, n-1$, such that if $i$ is zero or even, it follows that

$$
\mathbf{H F}^{i}=\left[\begin{array}{cccc}
\boldsymbol{\rho}_{11}^{T} \mathbf{G}_{1}^{i / 2} / \rho_{11} & \mathbf{0}^{T} & \mathbf{0}^{T} & \mathbf{0}^{T} \\
\boldsymbol{\rho}_{21}^{T} \mathbf{G}_{1}^{i / 2} / \rho_{21} & \mathbf{0}^{T} & \mathbf{0}^{T} & \mathbf{0}^{T} \\
\boldsymbol{\rho}_{31}^{T} \mathbf{G}_{1}^{i / 2} / \rho_{31} & \mathbf{0}^{T} & \mathbf{0}^{T} & \mathbf{0}^{T} \\
\mathbf{0}^{T} & \boldsymbol{\rho}_{12}^{T} \mathbf{G}_{2}^{i / 2} / \rho_{12} & \mathbf{0}^{T} & \mathbf{0}^{T} \\
\mathbf{0}^{T} & \boldsymbol{\rho}_{22}^{T} \mathbf{G}_{2}^{i / 2} / \rho_{22} & \mathbf{0}^{T} & \mathbf{0}^{T} \\
\mathbf{0}^{T} & \boldsymbol{\rho}_{32}^{T} \mathbf{G}_{2}^{i / 2} / \rho_{32} & \mathbf{0}^{T} & \mathbf{0}^{T} \\
\hline \hline-\boldsymbol{\rho}_{12}^{T} \mathbf{G}_{1}^{i / 2} / \rho_{12} & \boldsymbol{\rho}_{12}^{T} \mathbf{G}_{2}^{i / 2} / \rho_{12} & \mathbf{0}^{T} & \mathbf{0}^{T}
\end{array}\right]
$$


and if $i$ is odd, it follows that

$$
\mathbf{H F}^{i}=\left[\begin{array}{cccc}
\mathbf{0}^{T} & \mathbf{0}^{T} & \boldsymbol{\rho}_{11}^{T} \mathbf{G}_{1}^{(i-1) / 2} / \rho_{11} & \mathbf{0}^{T} \\
\mathbf{0}^{T} & \mathbf{0}^{T} & \boldsymbol{\rho}_{21}^{T} \mathbf{G}_{1}^{(i-1) / 2} / \rho_{21} & \mathbf{0}^{T} \\
\mathbf{0}^{T} & \mathbf{0}^{T} & \boldsymbol{\rho}_{31}^{T} \mathbf{G}_{1}^{(i-1) / 2} / \rho_{31} & \mathbf{0}^{T} \\
\mathbf{0}^{T} & \mathbf{0}^{T} & \mathbf{0}^{T} & \boldsymbol{\rho}_{12}^{T} \mathbf{G}_{2}^{(i-1) / 2} / \rho_{12} \\
\mathbf{0}^{T} & \mathbf{0}^{T} & \mathbf{0}^{T} & \boldsymbol{\rho}_{22}^{T} \mathbf{G}_{2}^{(i-1) / 2} / \rho_{22} \\
\mathbf{0}^{T} & \mathbf{0}^{T} & \mathbf{0}^{T} & \boldsymbol{\rho}_{32}^{T} \mathbf{G}_{2}^{(i-1) / 2} / \rho_{32} \\
\hline \hline \mathbf{0}^{T} & \mathbf{0}^{T} & -\boldsymbol{\rho}_{12}^{T} \mathbf{G}_{1}^{(i-1) / 2} / \rho_{12} & \boldsymbol{\rho}_{12}^{T} \mathbf{G}_{2}^{(i-1) / 2} / \rho_{12}
\end{array}\right]
$$

Equations (4.25)-(4.27) for Case 1.0 can easily be obtained by removing the relative measurement rows below the double line.

Once again it should be noted that the terms containing $\mathbf{G}$ are relatively small. In particular, since the spacecraft are in circular orbits such that $\left\|\mathbf{r}_{j}\right\|=$ $R_{\S}+100 \mathrm{~km}$, it follows that $\|\mathbf{G}\|=1.58 \times 10^{-6} 1 / \mathrm{s}^{2}$. Additionally, due to the increased dimension of the state, the observability matrix now contains powers of $\mathbf{G}$ which leads to small contributions. Consequently, these terms are again considered negligible when examining the observability matrix analytically. This implies that $\mathcal{O}$ approximately only contains the rows resulting from $\mathbf{H}$ and HF. However, this simplification is not made when computing $\operatorname{det}\left(\mathcal{O}^{T} \mathcal{O}\right)^{1 / 2}$ numerically.

First consider the terms $\mathbf{H}$ and $\mathbf{H F}$ for Case 1.0 (inertial measurements only). If any inertial range vectors to the same ${ }^{1}$ spacecraft are parallel, the corresponding set of rows of the observability matrix is linearly dependent.

\footnotetext{
${ }^{1}$ The inertial range vectors must be to the same spacecraft because inertial range terms for different spacecraft are contained in different columns of the observability matrix; consequently rows involving different spacecraft are always linearly independent.
} 
This implies that the set contributes only one linearly independent row. With the addition of a third tracking station this result can be further extended. Since any two linearly independent vectors in $\mathbb{R}^{3}$ form the basis for a plane, any other vector that lies in this plane is linearly dependent on the basis vectors [35]. Consequently, if three or more inertial range vectors to the same spacecraft lie in the same plane, the corresponding set of rows of the observability matrix is linearly dependent. This implies that the set contributes only two linearly independent rows.

Now reconsider the terms $\mathbf{H}$ and $\mathbf{H F}$ for Case 1.1 (fused relative measurement). Now notice that if any inertial range vectors to two different ${ }^{2}$ spacecraft are parallel, and the relative range vector between these two spacecraft is also parallel, then the corresponding set of rows of the observability matrix is linearly dependent. This implies that this set of three contributes only two linearly independent rows. In other words this particular relative measurement is not contributing to the observability of the state. This result can also be extended. Suppose that inertial range vectors from two stations to one spacecraft form a plane; and, two more inertial range vectors to a different spacecraft form another plane. If the two planes are coplanar the relative range vector between these two spacecraft must also lie in this plane because it has a point in each plane (located at each spacecraft). Therefore, the corresponding

\footnotetext{
${ }^{2}$ The inertial range vectors must now be to two different spacecraft because relative range (between these two spacecraft) terms occur in the same columns of the observability matrix as the inertial range terms of the two spacecraft; consequently two different spacecraft are required in order to span the columns of the rows containing relative range terms.
} 
set of rows of the observability matrix is linearly dependent. Similarly, if these two planes are not coplanar, but the relative range vector between these two spacecraft lies along the intersection of these two planes, then the corresponding set of rows of the observability matrix is again linearly dependent. In both instances the set of five contributes only four linearly independent rows. Once again this implies that this particular relative measurement is not contributing to the observability of the state.

Figures 4.7 and 4.8 illustrate the detection of these events both numerically and geometrically. In these figures the colored markings denote events occurring at the same respective times. In other words, the times where the colored points occur in Figure 4.7 have also been marked on the black trajectory in Figure 4.8. However, although the detection of these events may cause reductions in observability, they do not necessarily indicate a complete loss of observability (i.e. the number of linearly independent rows of the observability matrix may still be $n$ ). This is especially important to remember considering the simplification made that rows containing $\mathbf{G}$ are negligible.

Recall that all three tracking stations lie in the same plane $\left(X_{I^{-}} Y_{I}\right.$ plane). If the trajectory of the spacecraft also remained in the $X_{I}-Y_{I}$ plane all range vectors would lie in the same plane for all time and according to the geometric analysis $\left(\mathbf{G}_{j} \approx \mathbf{0}\right)$ the observability condition would be violated for all time and the z-component of position would be unobservable. It is no coincidence that this mission segment examines a polar orbit. Since the orbit is perpendicular to the $X_{I^{-}} Y_{I}$ plane it allows for maximum out-of-plane $\left(Z_{I}\right)$ 


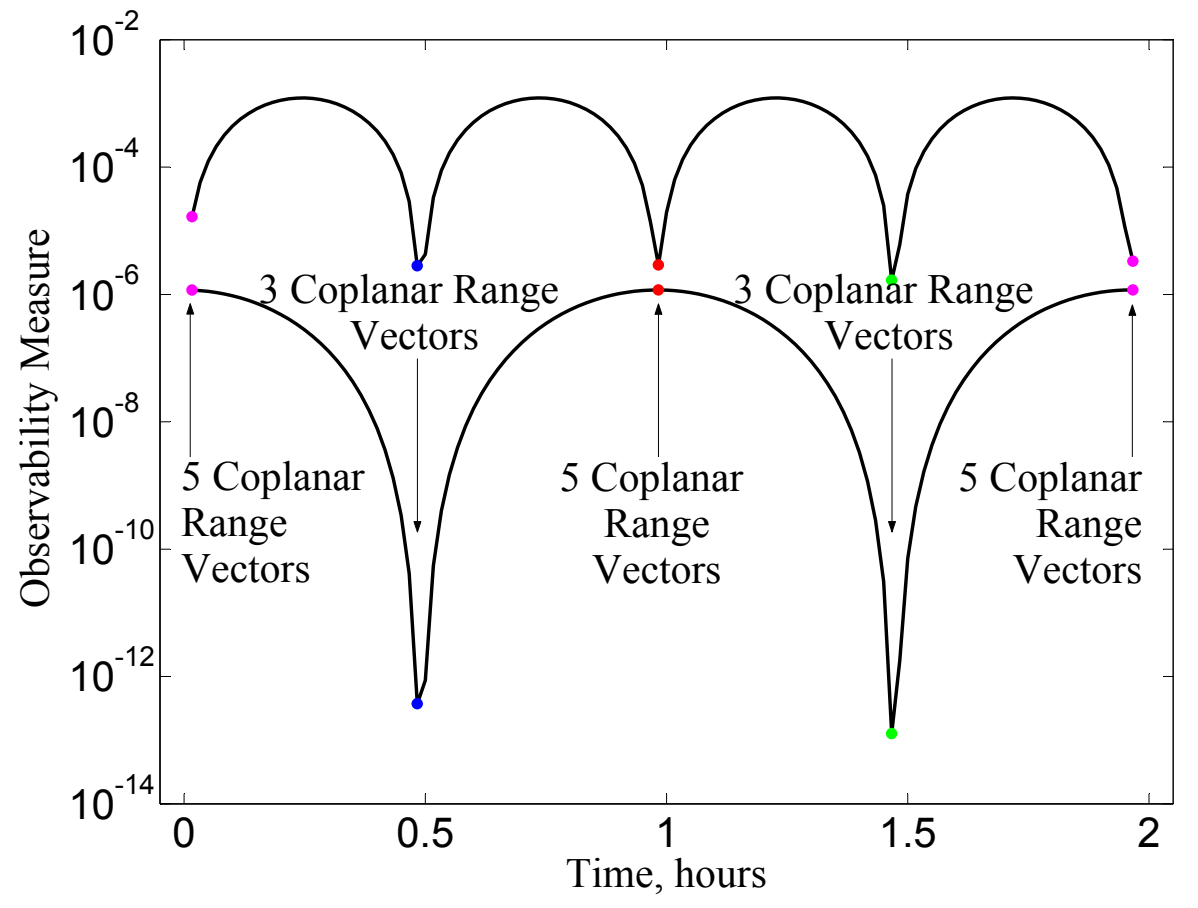

Figure 4.7: Numerical detection for Case 1.0 (lower curve) and Case 1.1 (upper curve).

position for a given altitude. However, despite the chosen trajectory, there are still two points of concern. First, as seen in Figure 4.7 near times 0.5 hours and 1.5 hours, there is still a considerable reduction in observability when the trajectory crosses the $X_{I^{-}} Y_{I}$ plane. Second, since the tracking stations are distant relative to the distance that the trajectory travels out of plane, the inertial range vectors nearly lie in the same plane. Consequently, the value of $\operatorname{det}\left(\boldsymbol{O}^{T} \boldsymbol{O}\right)^{1 / 2}$ remains small relative to that of the cislunar simulation. 


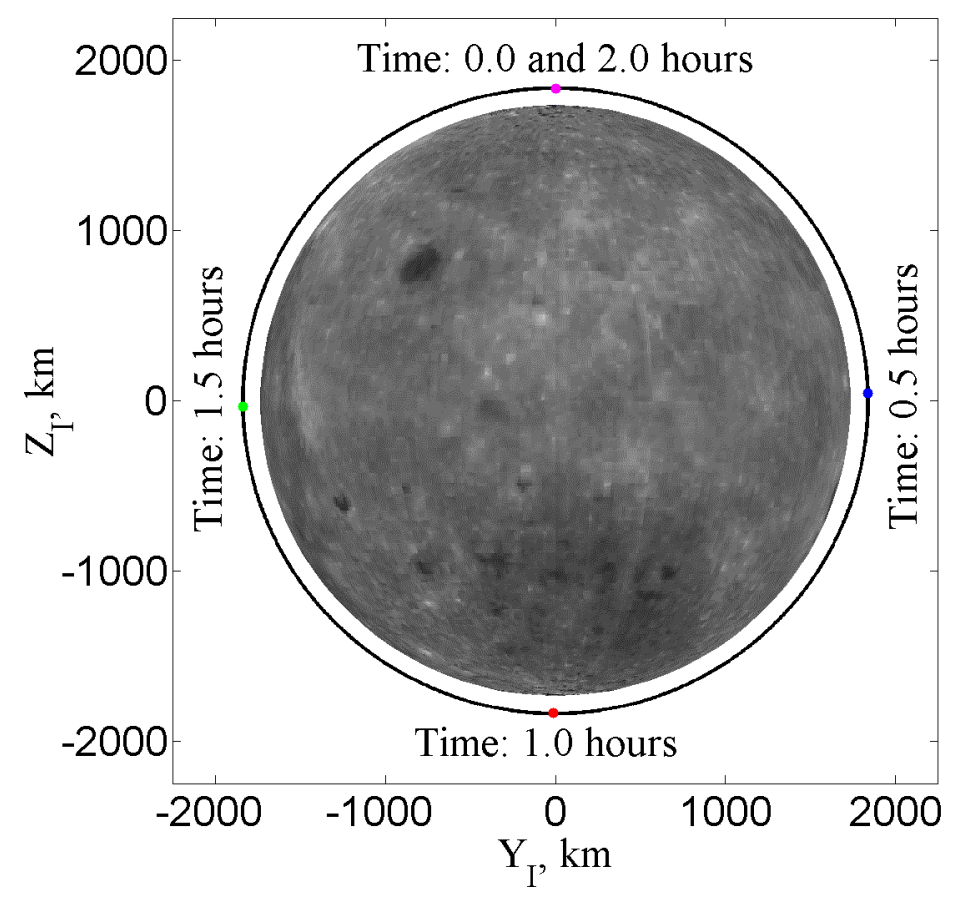

Figure 4.8: Geometrical arrangement for Case 1.

Figure 4.7 illustrates the increase in observability due to the addition of a relative measurement. ${ }^{3}$ Notice that when the relative measurement is included the observability measure is always greater than it is without the relative measurement. Also, notice that near times 0 hours, 1 hour, and 2 hours the observability measure for both Cases 1.0 and 1.1 is roughly the same.

\footnotetext{
${ }^{3}$ Recall from Section 2.3.1 that tracking station visibility blackouts have been ignored. When these blackouts are incorporated, during the portions of the trajectory in which the number of stations is reduced (as indicated by Figure 2.6), both observability measure curves are shifted downward several orders of magnitude.
} 


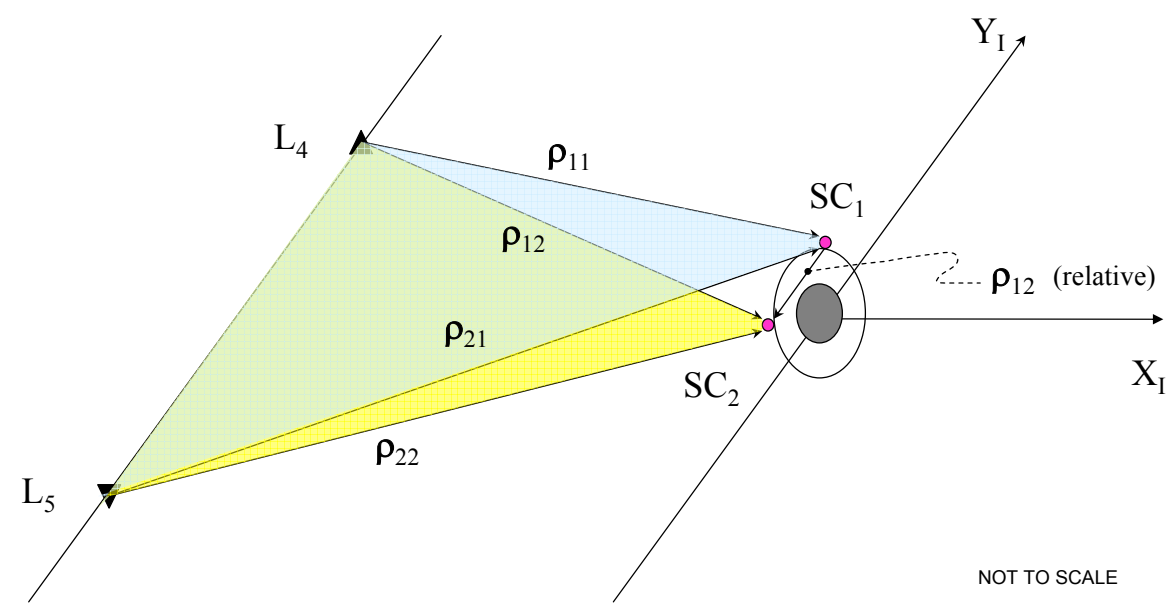

Figure 4.9: Geometrical detection for Case 1.

This is expected because of the aforementioned event occurring at these times. Namely, the event in which the planes created by the inertial range vectors to two spacecraft are coplanar, as seen in Figure 4.9. Notice that these events occur when both spacecraft are near the "top" and "bottom" of the orbit. To be more exact, the relative range vector between the two spacecraft is

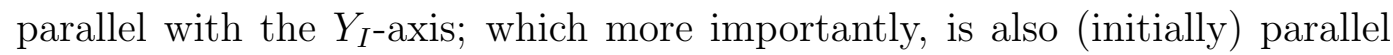
with a line connecting the $L_{4}$ and $L_{5}$ tracking stations. This implies that the plane created by the $L_{4}$ and $L_{5}$ inertial range vector to the first spacecraft is (nearly) coplanar with the plane created by the $L_{4}$ and $L_{5}$ inertial range vector to the second spacecraft. Therefore, as described earlier, the relative range measurement row in the observability matrix is a linear combination 
of the four tracking station rows. Consequently, the relative measurement contributes no additional information and Case 1.1 is essentially reduced to Case 1.0.

As expected all residuals are again approximately zero-mean, white noise with a standard deviation of about $5 \mathrm{~m}$, similar to what was seen in Figure 4.6. The navigation state error for the first spacecraft in Case 1.0, can be seen in Figures 4.10 and 4.11. The bulges in the state estimate error covariance occurring just before 1.5 hours roughly coincide with the violation of the observability condition, as seen in Figure 4.7. Also notice that the largest position errors are in the $Z_{I}$ direction. This is not surprising considering that all the inertial tracking stations are located in the $X_{I^{-}} Y_{I}$ plane. Consequently, as discussed later, the out-of-plane ability of the relative measurement yields the largest reduction in the navigation error of the $Z_{I}$ position component.

Plots for the second spacecraft, as well as for Case 1.1, are not presented here as they look very similar to Figures 4.10 and 4.11. Despite this similarity, by examining the state error covariance that bounds the state error at the final time for Cases 1.0 and 1.1, as seen in Table 4.2, notice that there is slight reduction in navigation error due to fusing the relative measurements. In particular there is roughly a $15 \%$ reduction in the $y$ and $\dot{z}$ components, roughly a $25 \%$ reduction in the $z$ and $\dot{y}$ components, but essentially no change in the $x$ and $\dot{x}$ components. These reductions are relatively small for a few reasons. First, only one relative measurement is available. As will be shown later, additional relative measurements can lead to larger reductions in navigation 

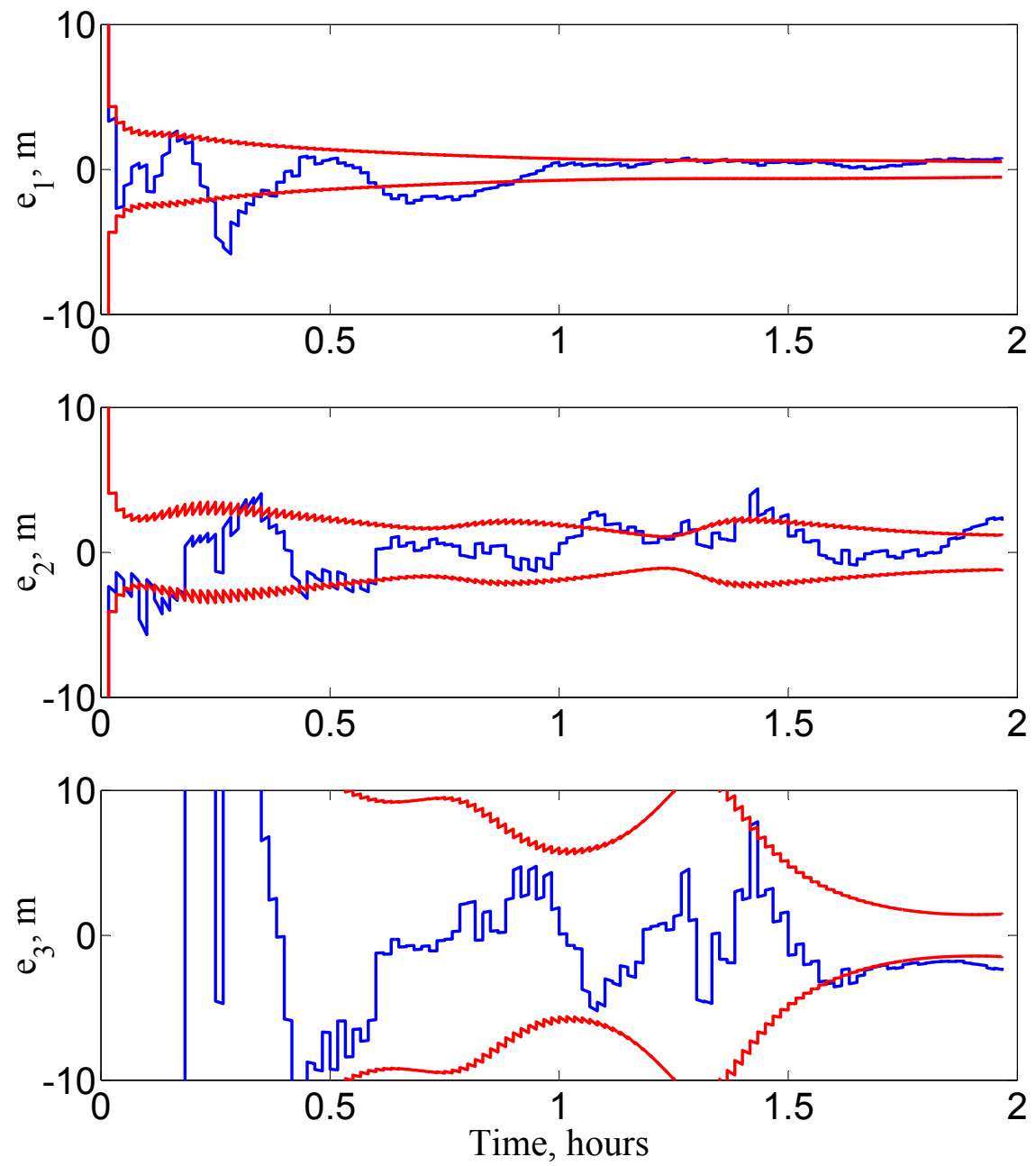

Figure 4.10: Time history of the inertial position errors for Case 1.0.

error. Second, since three inertial measurements are used there is already a high degree of observability without the relative measurements. Consequently, 

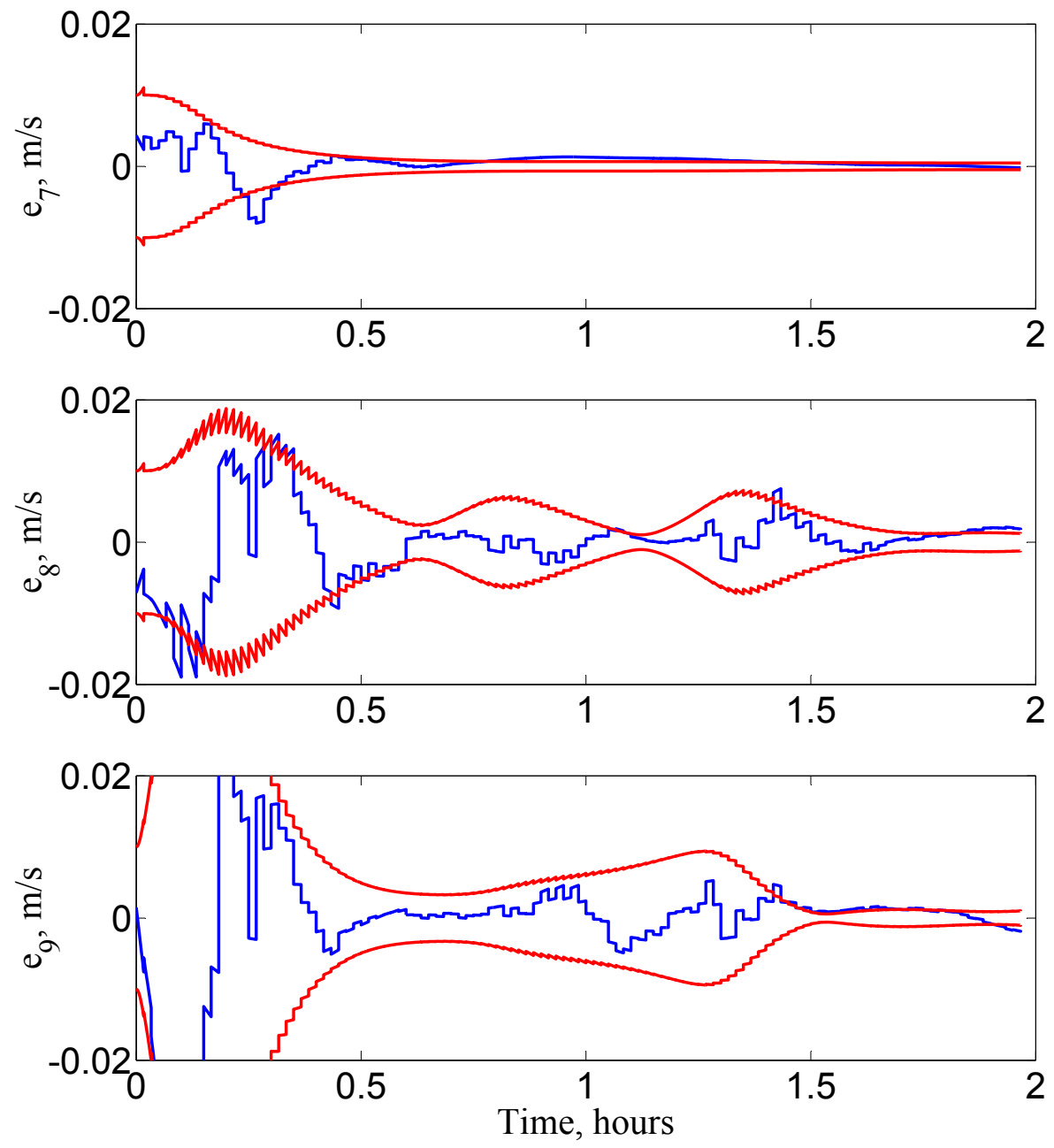

Figure 4.11: Time history of the inertial velocity errors for Case 1.0.

the navigation error is mostly driven by the inertial measurements. However, as presented in [28], a four spacecraft formation using only one inertial mea- 
surement can achieve about a 50\% reduction in the navigation errors of all components when fusing the six available relative measurements. Finally, the lack of change of the components in the $X_{I^{-}}$-direction is an expected result of the geometry. Since all the relative measurements are in the $Y_{I^{-}} Z_{I}$ plane, they do not contribute any information about the $X_{I^{-}}$-direction.

Case 1 illustrates how the geometry of a problem can affect observability. It also demonstrates that relative measurements can be used to decrease inertial navigation error. Case 2 extends this concept further by examining the advantages and complexities of using multiple relative measurements. For Case 2, the system dynamics matrix $\mathbf{F}$ and the measurement mapping matrix $\mathbf{H}$ are defined using Eqs. (2.20) and (2.26), respectively. While the full measurement mapping matrix and observability matrix $\mathcal{O}$ for Case 2 are too large to present here, they are very similar to those described in Eqs. (4.25) to (4.27) for Case 1. Moreover, the Case 2 observability matrix shares all of the reduced observability events presented in Case 1. Likewise, due to the magnitude of $\|\mathbf{G}\|$ for simplification terms containing $\mathbf{G}$ are again considered negligible when examining the observability matrix analytically. This again implies that $\mathcal{O}$ essentially only contains the rows resulting from $\mathbf{H}$ and $\mathbf{H F}$.

Examining $\mathbf{H}$ shows that the addition of multiple relative measurements yields an additional reduced observability event. In particular, consider the rows containing relative measurement terms between any three spacecraft. If any two relative range vectors between these three spacecraft are parallel, the third must also be parallel (i.e. the spacecraft are collinear). Consequently, 
the corresponding set of rows of the observability matrix is linearly dependent. Therefore this set of three contributes only two linearly independent rows, which implies that one of the relative measurements is not contributing to the observability of the state.

While the formation in Case 2 was chosen such that this event does not occur, the events described in Case 1 still do occur. The numerical detection of these events in Case 2 are illustrated in Figure 4.12; while since the additional planes of Case 2 are nearly coplanar with the plane of Case 1, Figure 4.8 is still valid for the geometrical detection. (For more information on the formation geometries of Cases 1 and 2 see Section 2.3.1).

Figure 4.12 illustrates the dramatic increase in observability due to the relative measurements. ${ }^{4}$ Notice that the addition of multiple relative measurements now compensates for the loss of observability during the $X_{I}-Y_{I}$ plane crossing. Also, while reductions in observability still do occur near the "top" and "bottom" of the orbits, as in Case 1.1, Case 2.1 is never fully reduced to Case 2.0 because of the use of multiple relative measurements.

Again, as expected all the residuals are approximately zero-mean, white noise with a standard deviation of about $5 \mathrm{~m}$, similar to what was seen in Figure 4.6. The navigation state error plots for Case 2.0 are not presented here as they look very similar to what was seen in Figures 4.10 and 4.11. In

\footnotetext{
${ }^{4}$ Recall from Section 2.3.1 that tracking station visibility blackouts have been ignored. When these blackouts are incorporated, during the portions of the trajectory in which the number of stations is reduced (as indicated by Figure 2.6), both observability measure curves are shifted downward several orders of magnitude.
} 


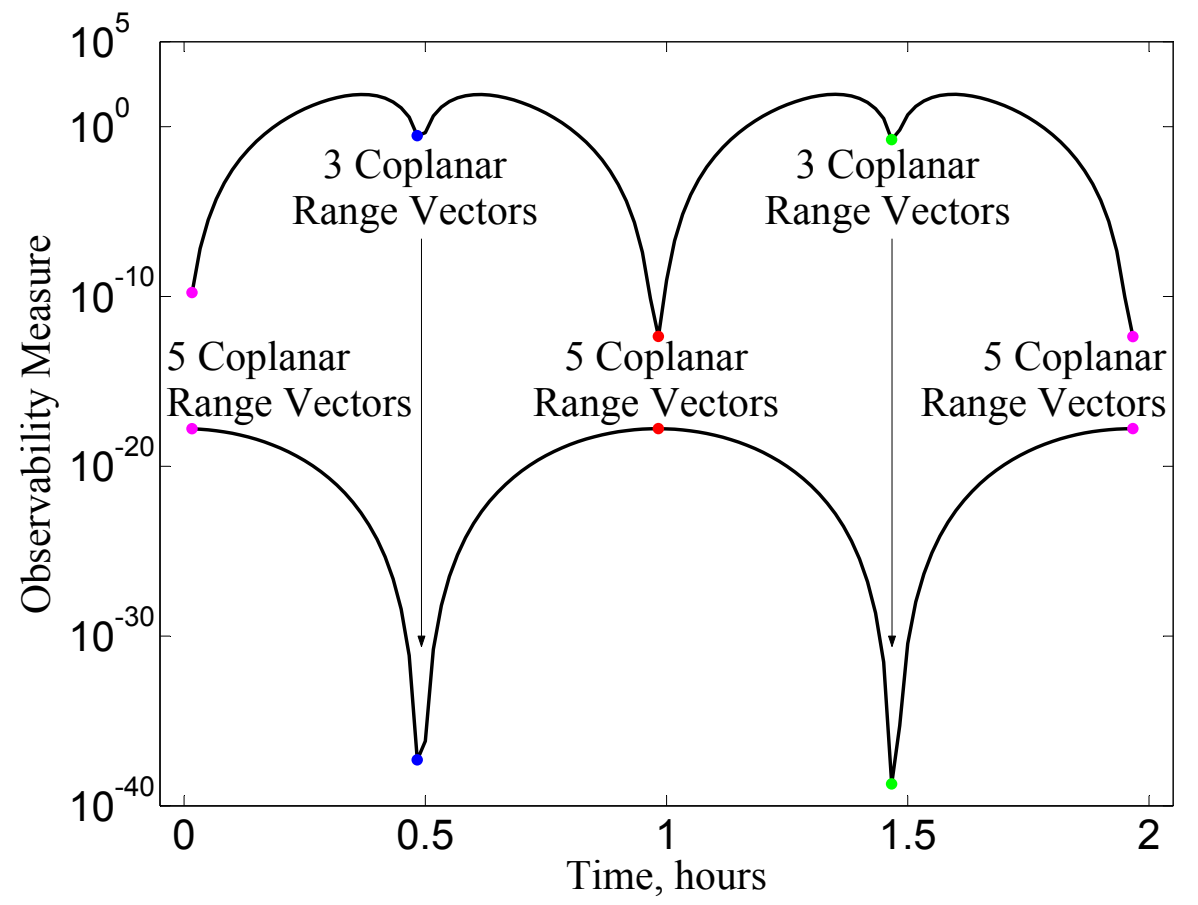

Figure 4.12: Numerical detection for Case 2.0 (lower curve) and Case 2.1 (upper curve).

fact, as seen in Table 4.2, the average of the final state error covariance for all six spacecraft for Case 2.0 is essentially the same as Case 1.0. This is to expected though since without the use of relative measurements the navigation of each spacecraft is essentially an independent, yet similar problem. When the relative measurements are added however, the reduction in navigation error for Case 2.1 is quite noticeable. In particular, Table 4.2 shows roughly a $55 \%$ reduction in the $z$ and $\dot{y}$ components and roughly a $30 \%$ reduction in all other components. Also, notice that unlike Case 1.1, Case 2.1 has a reduction in the 

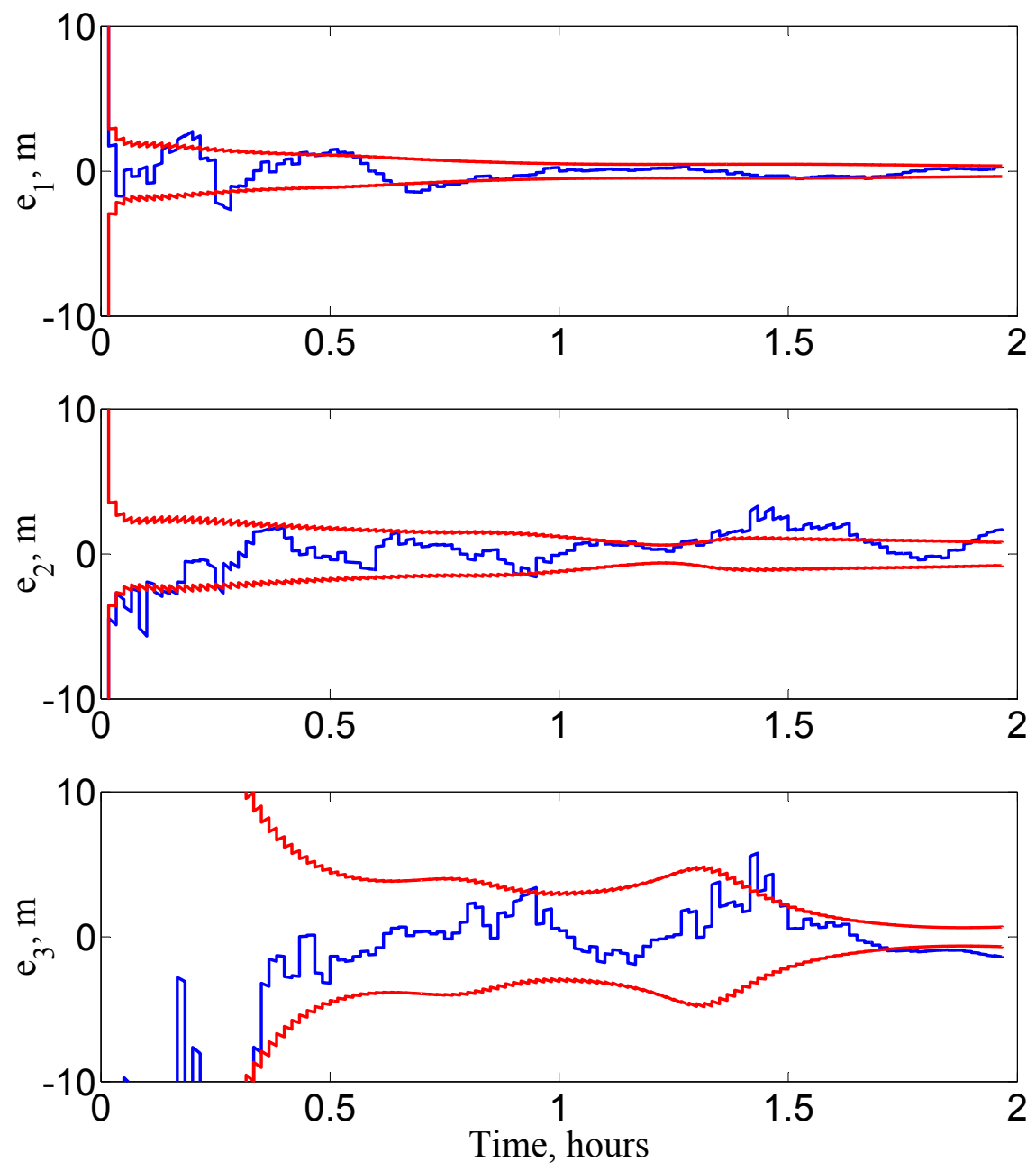

Figure 4.13: Time history of the inertial position errors for Case 2.1.

$X_{I}$-direction. This reduction in the $X_{I}$-direction is the direct result of relative measurements taken out of the $Y_{I^{-}} Z_{I}$ plane. 

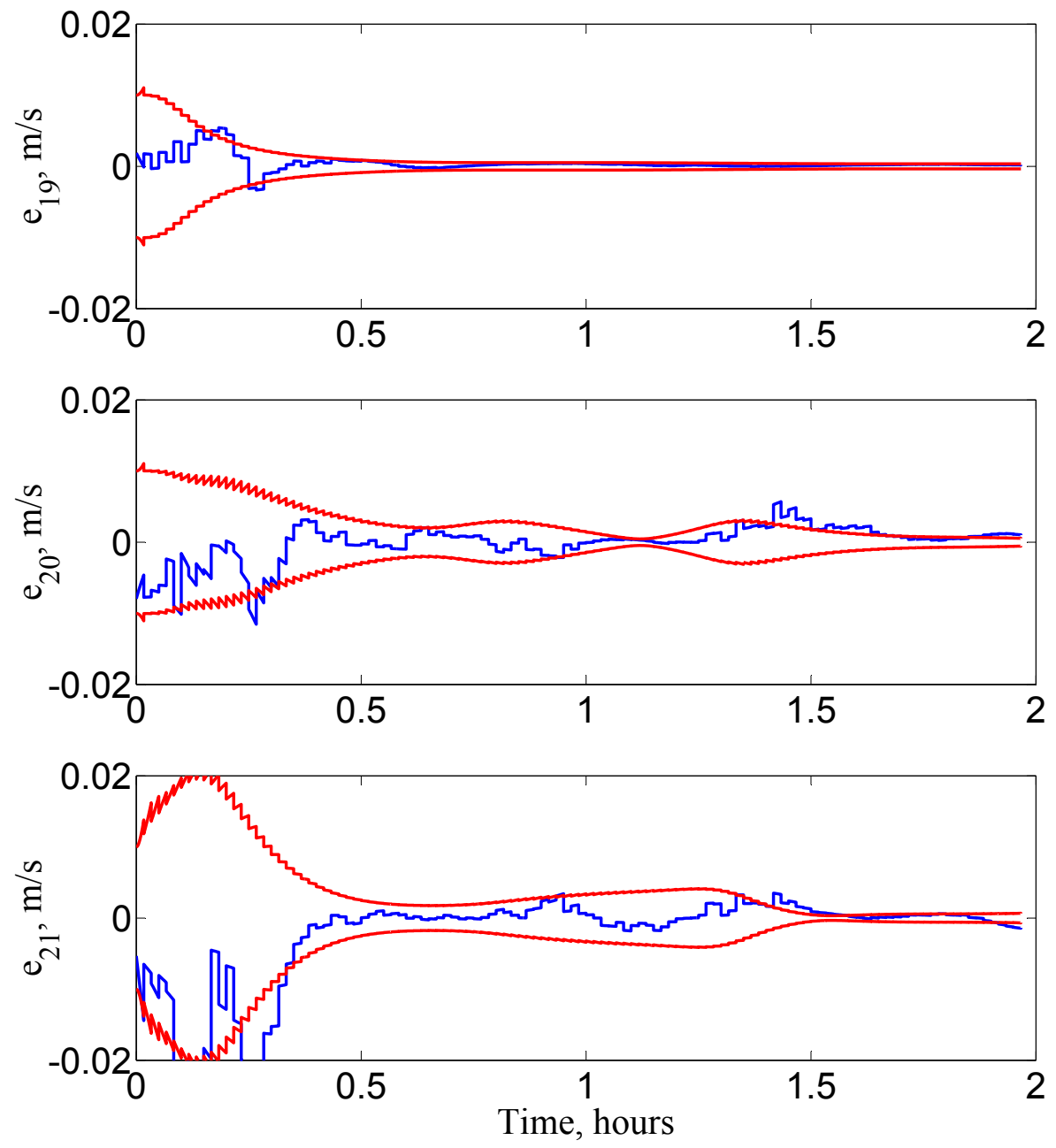

Figure 4.14: Time history of the inertial velocity errors for Case 2.1.

Also, as seen in Figures 4.13 and 4.14, there is an overall reduction in the magnitudes of the state estimate error covariance bulges, and an apparent 
increase in the rate of convergence, especially in the $z$ and $\dot{y}$ components. In particular, for the $z$ components covariance to converge to less than the measurement noise of $5 \mathrm{~m}$, Cases 1.0 and 2.0 required 90 measurement updates, Case 1.1 required 54 measurement updates, and Case 2.1 required only 28 measurement updates (updates occur every minute).

Finally, the complete navigation performance for Cases 1 and 2 at a final time of $t_{f}=t_{o p}$ is summarized in Table 4.2. The over bar on the state error covariance $P$ indicates that the values shown are the average values of all the spacecraft. This was done since the state error covariance history of each spacecraft is practically identical.

Table 4.2: Mission Cases Performance Comparison

\begin{tabular}{ccccccc}
\hline \hline Case & $\begin{array}{c}\bar{P}_{x x}^{1 / 2}\left(t_{f}\right) \\
(\mathrm{m})\end{array}$ & $\begin{array}{c}\bar{P}_{y y}^{1 / 2}\left(t_{f}\right) \\
(\mathrm{m})\end{array}$ & $\begin{array}{c}\bar{P}_{z z}^{1 / 2}\left(t_{f}\right) \\
(\mathrm{m})\end{array}$ & $\begin{array}{c}\bar{P}_{\dot{x} \dot{x}}^{1 / 2}\left(t_{f}\right) \\
(\mathrm{mm} / \mathrm{s})\end{array}$ & $\begin{array}{c}\bar{P}_{\dot{y} \dot{y}}^{1 / 2}\left(t_{f}\right) \\
(\mathrm{mm} / \mathrm{s})\end{array}$ & $\begin{array}{c}\bar{P}_{\dot{z} \dot{z}}^{1 / 2}\left(t_{f}\right) \\
(\mathrm{mm} / \mathrm{s})\end{array}$ \\
\hline 1.0 & 0.532 & 1.161 & 1.450 & 0.473 & 1.219 & 0.977 \\
1.1 & 0.531 & 0.974 & 1.067 & 0.473 & 0.890 & 0.816 \\
2.0 & 0.532 & 1.161 & 1.450 & 0.473 & 1.218 & 0.977 \\
2.1 & 0.363 & 0.800 & 0.675 & 0.353 & 0.550 & 0.668 \\
\hline \hline
\end{tabular}

\subsubsection{Constant Formation Geometry}

Consider using the analysis of the previous sections to work the problem in reverse. In other words, instead of examining a given geometry for possible observability deficiencies, suppose instead the maximum of the observability measure is analyzed to determine the optimal navigation geometry.

To illustrate this, consider the observability of a formation based solely 
on the relative measurements between each spacecraft. More specifically suppose the formation is such that the relative position between each spacecraft remains constant. Thus, although the orientation of this formation may rotate in inertial space, there exists a frame fixed to the formation in which the position of each spacecraft remains constant (but unknown). Since in the formation-fixed frame the velocity of each spacecraft is always zero, the state in this frame need only consist of position and $\mathbf{F}=\mathbf{0}$. This lack of dynamics then results in $\boldsymbol{O}^{T} \boldsymbol{O}=\mathbf{H}^{T} \mathbf{H}$, which in turn implies that to satisfy the LTI observability condition $m \geq n$. Consequently, since $m=p(p-1) / 2$ and $n=3 p$ (in three dimensions), this requires that $p \geq 7$.

Fortunately, it turns out that this can be avoided by noting the geometry of the problem. First, since the orientation of the formation-fixed frame $\left(X_{F^{-}} Y_{F^{-}} Z_{F}\right)$ is completely arbitrary, suppose the frame is placed such that the $X_{F}$-axis points from Spacecraft 1 to Spacecraft 2 . Next, since the placement of the origin is also arbitrary let it be placed halfway between Spacecraft 1 and Spacecraft 2. Thus, since the remaining distances can be normalized by the distance between Spacecraft 1 and Spacecraft 2, the locations of the first two spacecraft are now known in this frame. Finally, the $Y_{F}$-axis is defined such that it lies in the plane created by Spacecraft 1, 2, and 3. Thus, since the state now need only consist of the $X_{F^{-}} Y_{F}$ position components of Spacecraft 3 and the $X_{F^{-}} Y_{F^{-}} Z_{F}$ position components of the remaining spacecraft, the dimension of the state-space is now $n=2+3(p-3)$ (in three dimensions), which meets the requirement that $m \geq n$. 
Using this choice for the formation-fixed frame the position of the first three spacecraft, which have a $Z_{F}$ position component equal to zero in three dimensions, are defined in two dimensions as follows:

$$
\mathbf{r}_{1}=\left[\begin{array}{c}
-0.5 \\
0
\end{array}\right] \quad, \quad \mathbf{r}_{2}=\left[\begin{array}{c}
0.5 \\
0
\end{array}\right] \quad, \quad \mathbf{r}_{3}=\left[\begin{array}{l}
x \\
y
\end{array}\right]
$$

Defining the state to be $\mathbf{x}=\mathbf{r}_{3}$, the measurement model and measurement mapping matrix are given by

$$
\mathbf{h}=\left[\begin{array}{c}
\rho_{12} \\
\rho_{13} \\
\rho_{23}
\end{array}\right] \quad, \quad \mathbf{H}=\left[\begin{array}{c}
\mathbf{0}^{T} \\
\boldsymbol{\rho}_{13}^{T} / \rho_{13} \\
\boldsymbol{\rho}_{23}^{T} / \rho_{23}
\end{array}\right]
$$

where $\rho_{i j}=\left\|\boldsymbol{\rho}_{i j}\right\|$ and $\boldsymbol{\rho}_{i j}=\mathbf{r}_{j}-\mathbf{r}_{i}$. Using Eq. (4.29), the performance index $J$ is then defined as,

$$
J=\operatorname{det}\left(\boldsymbol{O}^{T} \boldsymbol{O}\right)=\operatorname{det}\left(\mathbf{H}^{T} \mathbf{H}\right)=\frac{16 y^{2}}{\alpha}
$$

where

$$
\alpha:=16 x^{4}-8 x^{2}+32 x^{2} y^{2}+8 y^{2}+16 y^{4}+1
$$

Since the Gram matrix $\mathcal{O}^{T} \mathcal{O}$ is always positive semidefinite, its determinant is always greater than or equal to zero, which implies $J \geq 0$. Notice then that according to Definition 4.1.1, the minimum value of $J$ corresponds to a violation of the LTI observability condition. With this in mind, it is desirable to determine the values of $x$ and $y$ that maximize (or minimize) $J$. This in turn requires examination of the $x$ - $y$ pairs that cause $J_{x}=0$ and $J_{y}=0$, where

$$
\begin{aligned}
& J_{x}=\frac{\partial J}{\partial x}=-\frac{256 y^{2} x}{\alpha^{2}}\left(4 x^{2}+4 y^{2}-1\right) \\
& J_{y}=\frac{\partial J}{\partial y}=-\frac{256 y^{3}}{\alpha^{2}}\left(4 x^{2}+4 y^{2}+1\right)+\frac{32 y}{\alpha}
\end{aligned}
$$


The most apparent root of Eq. (4.32) is the solution $y=0$. Evaluating $J$ along this solution results in the minimization of the performance index $(J=0)$ and the violation of the LTI observability condition. Also notice that the $y=0$ solution corresponds to the three spacecraft being collinear, which recall was also noted as a deficient geometry in Section 4.2.2. Next, considering solutions such that $x=0$ but $y \neq 0$, Eq. (4.32) reduces to the solution $y= \pm 0.5$. Expanding this solution such that $x \neq 0$ results in the root equation $x^{2}+y^{2}=0.5^{2}$. Thus, combining these two results it turns out that the location of the third spacecraft that maximize $J$ is anywhere on a circle of radius 0.5 , except $y=0$. This is illustrated in Figure 4.15 , where the green dots represents ideal locations for Spacecraft $3\left(S C_{3}\right)$, while the black and blue dots represent the locations of Spacecraft 1 and $2\left(S C_{1}\right.$ and $\left.S C_{2}\right)$, respectively. An interesting side note about this formation is that although an alternative line of reasoning is used, the end result is somewhat analogous to the findings of Bishop and Nabaa [5], which show that the optimal two-dimensional sensor placement strategy (that minimizes the least-squares covariance) is a circle of smallest possible radius.

It should be noted at this point that to definitively prove that these solutions represent maximums requires the examination of higher-order derivatives of $J$. Since even for this simple example this analysis becomes quite involved, consider instead the numerical evaluation of $J$ for $x-y$ pairs on a (rectilinear or polar) grid of resolution 1/100. As shown in Figure 4.16, the maximum value of $J=1$ is obtained along a circle of radius 0.5 . 


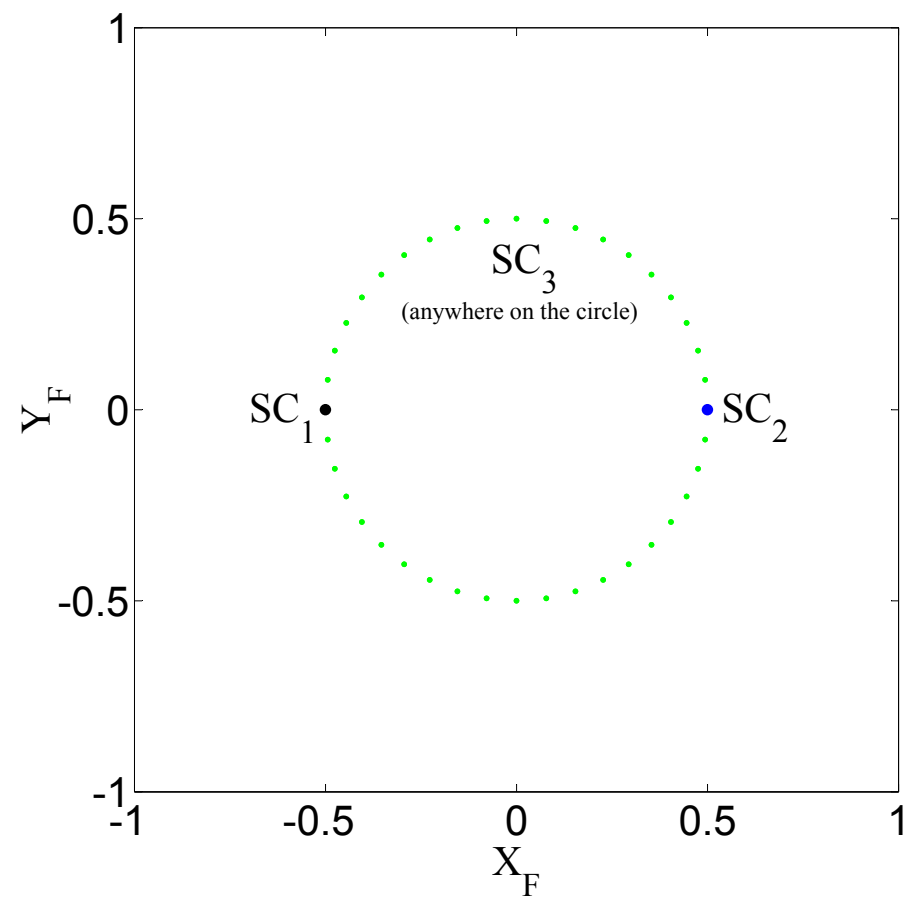

Figure 4.15: Ideal three spacecraft formation in two dimensions.

Using this numerical grid search technique insight can be gained into the possible optimal formation for more complicated problems. For example, consider the previous two-dimensional problem with the addition of a fourth spacecraft. To analyze this problem every point on the grid of the fourth spacecraft, must be evaluate for all points on the grid of the third spacecraft. Examining the resulting values for $J$ indicates that the local maximum on the grid occurs when $\mathbf{r}_{3}=\left[\begin{array}{ll}0.0 & 0.4\end{array}\right]^{T}$ and $\mathbf{r}_{4}=\left[\begin{array}{ll}0.0 & -0.4\end{array}\right]^{T}$. This is illustrated in Figure 4.17, where the black, blue, green, and red dots indicate Spacecraft 


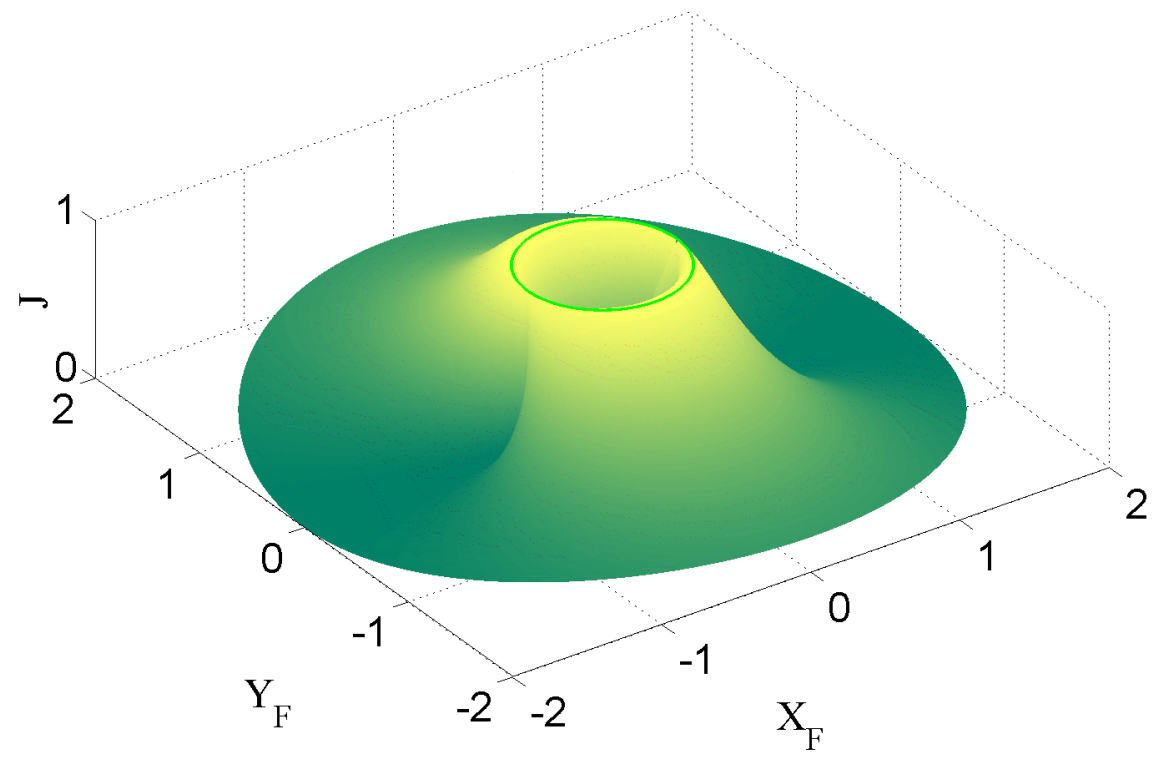

Figure 4.16: Evaluation of $J$ for three spacecraft in two dimensions.

1, 2, 3, and $4\left(S C_{1}, S C_{2}, S C_{3}\right.$, and $\left.S C_{4}\right)$, respectively. Also notice that, when a fourth spacecraft is introduced, all the spacecraft no longer lie on a circle of radius 0.5 .

As a final example, the four spacecraft analysis is expanded to three dimensions such that,

$$
\mathbf{r}_{3}=\left[\begin{array}{c}
x_{3} \\
y_{3} \\
0
\end{array}\right] \quad, \quad \mathbf{r}_{4}=\left[\begin{array}{c}
x_{4} \\
y_{4} \\
z_{4}
\end{array}\right] \quad, \quad \mathbf{x}=\left[\begin{array}{l}
x_{3} \\
y_{3} \\
x_{4} \\
y_{4} \\
z_{4}
\end{array}\right]
$$




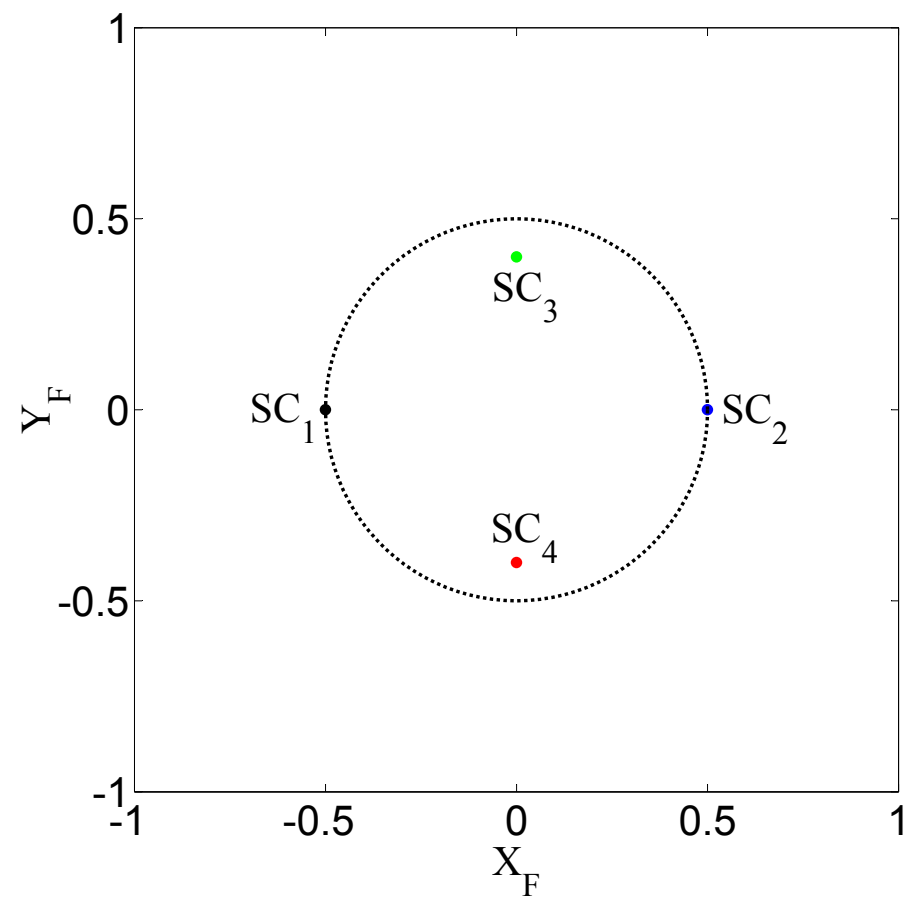

Figure 4.17: Proposed four spacecraft formation in two dimensions.

With the state defined, the measurement model and measurement mapping matrix are given by,

$$
\mathbf{h}=\left[\begin{array}{c}
\rho_{12} \\
\rho_{13} \\
\rho_{14} \\
\rho_{23} \\
\rho_{24} \\
\rho_{34}
\end{array}\right] \quad, \quad \mathbf{H}=\left[\begin{array}{cc}
\mathbf{0}^{T} & \mathbf{0}^{T} \\
\tilde{\boldsymbol{\rho}}_{13}^{T} / \rho_{13} & \mathbf{0}^{T} \\
\mathbf{0}^{T} & \boldsymbol{\rho}_{14}^{T} / \rho_{14} \\
\tilde{\boldsymbol{\rho}}_{23}^{T} / \rho_{23} & \mathbf{0}^{T} \\
\mathbf{0}^{T} & \boldsymbol{\rho}_{24}^{T} / \rho_{24} \\
-\tilde{\boldsymbol{\rho}}_{34}^{T} / \rho_{34} & \boldsymbol{\rho}_{34}^{T} / \rho_{34}
\end{array}\right]
$$

where the tilde accent $\left(^{\sim}\right)$ indicates that $\tilde{\boldsymbol{\rho}}_{i j}$ is a two-dimensional vector consisting only of the $x$ and $y$ components of $\boldsymbol{\rho}_{i j}$. Regardless of this reduction 
in dimension, notice in Eq. (4.34) that if all four spacecraft are coplanar, the last row of $\mathbf{H}$ can be written as a linear combination of the previous four rows (the first row is necessarily linear dependent). This then implies that the rank of $\mathbf{H}$ (four) will be less than the dimension of the state (five). Consequently evaluating $J$ for a three-dimensional formation of coplanar spacecraft results in the minimization of the performance index $(J=0)$ and the violation of the LTI observability condition (as seen in Figures 4.19 and 4.20).

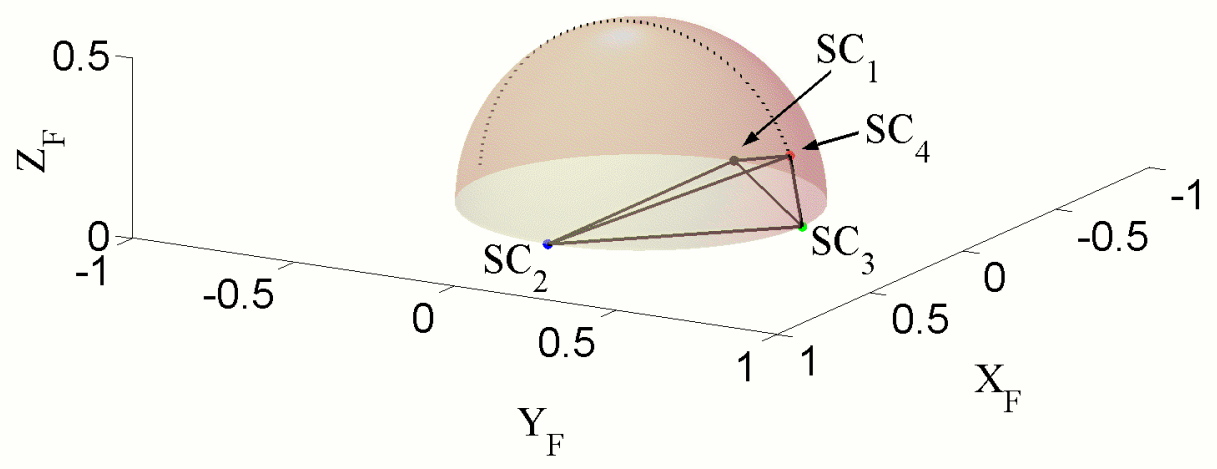

Figure 4.18: Proposed four spacecraft formation in three dimensions.

Returning to the numerical search technique, examining the resulting values for $J$ now indicates that the local maximum on the three-dimensional grid occurs when $\mathbf{r}_{3}=\left[\begin{array}{ll}0.0 & 0.5\end{array}\right]^{T}$ and $\mathbf{r}_{4}$ has a zero $X_{F}$ component and the $Y_{F}$ component is as close as possible to $\mathbf{r}_{3}$ along a perpendicular circle of radius 
0.5. This is illustrated in Figure 4.18 on a red hemisphere of radius 0.5 , where again the black, blue, green, and red dots indicate Spacecraft 1, 2, 3, and 4 $\left(S C_{1}, S C_{2}, S C_{3}\right.$, and $\left.S C_{4}\right)$, respectively. (Due to symmetry, the $\mathbf{r}_{3}$ search can be restricted to the positive $X_{F^{-}} Y_{F}$ quadrant and the $\mathbf{r}_{4}$ search can be restricted to positive $Z_{F}$ components. For visualization purposes a relatively large $Z_{F}$ component was chosen for Spacecraft 4 in Figure 4.18.)

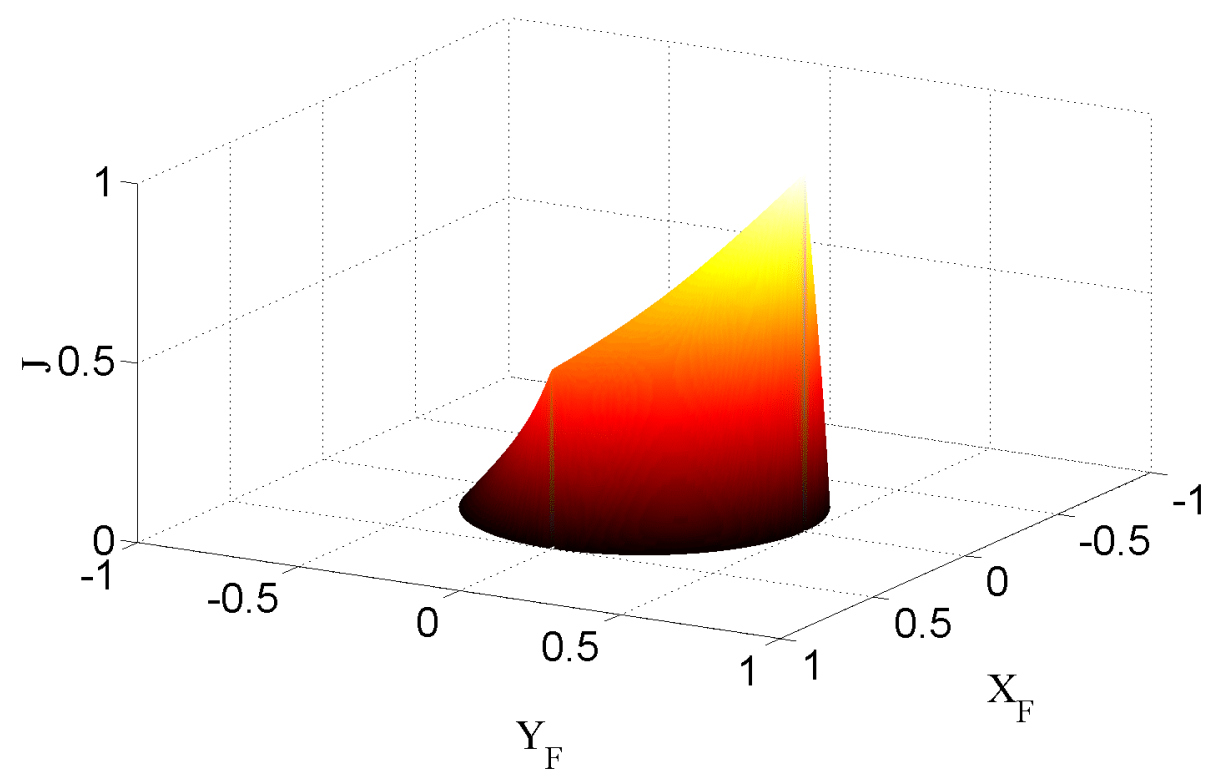

Figure 4.19: Evaluation of $J$ for four spacecraft restricted to a hemisphere.

Finally, for completeness, Figure 4.19 shows the numerical evaluation of $J$ for grid points corresponding to $\mathbf{r}_{3}=\left[\begin{array}{ll}0.0 & 0.5\end{array}\right]^{T}$ and $\mathbf{r}_{4}$ restricted to 
the red hemisphere of Figure 4.18. This can also be visualized in Figure 4.20 by mapping the color values of $J$ (0 to 1 ) in Figure 4.19 to their corresponding location on the hemisphere. Notice in both figures that the value of $J$ increases as Spacecraft 4 approaches Spacecraft 3.
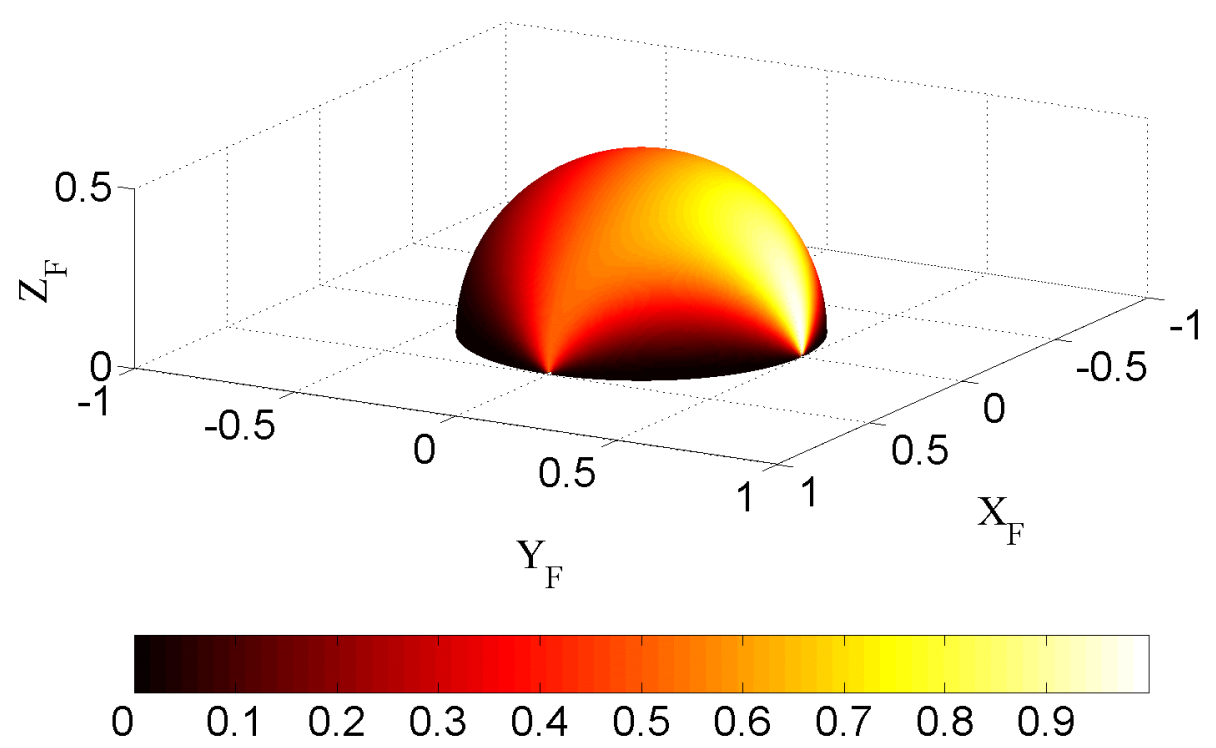

Figure 4.20: Evaluation of $J$ for four spacecraft restricted to a hemisphere.

\subsection{Observability-Error Covariance Relationship}

Once the impact of observer and formation geometry on the observability is understood, it is desirable to relate the observability with the state 
error covariance. In other words, if a particular geometry indicates a certain degree of observability, what is the corresponding level of achievable state estimation accuracy. First, Section 4.3.1 relates the LTI observability matrix to an upper bound on the state error covariance. Section 4.3.2 then describes the well established Cramér-Rao lower bound on the state error covariance and relates this bound to the observability Gramian. Finally, as will be shown in Section 4.3.3, under the right conditions, the resulting Gramian relationship is somewhat analogous to the approximate state error covariance forecasted using DOP metrics.

\subsubsection{LTI-Based Covariance Bound}

In their effort to determine an optimal sensor placement strategy, Bishop and Nabaa [5] present the relationship between the Kalman filter error covariance and the covariance of the basic weighted least-squares state estimate error, $\mathbf{P}_{L S}$, where

$$
\mathbf{P}_{L S}=\left(\mathbf{H}^{T} \mathbf{R}^{-1} \mathbf{H}\right)^{-1}
$$

In particular, by considering the Information Form of the Kalman filter [7],

$$
\mathbf{P}^{-1}\left(t_{d}^{+}\right)=\mathbf{P}^{-1}\left(t_{d}^{-}\right)+\mathbf{H}^{T}\left(t_{d}^{-}\right) \mathbf{R}^{-1}\left(t_{d}^{-}\right) \mathbf{H}\left(t_{d}^{-}\right)
$$

Bishop and Nabaa show that the information of the Kalman filter is larger than the information of the least-squares estimate since $\mathbf{P}^{-1}\left(t_{d}^{-}\right) \geq \mathbf{0}$ implies ${ }^{5}$

$$
\mathbf{P}^{-1}\left(t_{d}^{+}\right) \geq \mathbf{P}_{L S}^{-1}\left(t_{d}^{-}\right)
$$

\footnotetext{
${ }^{5}$ In general, the matrix inequality $\mathbf{A} \leq \mathbf{B}$ is equivalent to stating that $(\mathbf{B}-\mathbf{A})$ is positive semidefinite.
} 
Although the relationship between the estimation error covariance of the EKF and least-squares estimate is less clear (since they are linearized about different trajectories), when the EKF is operating nominally, the least-squares upper bound still offers a means of analyzing the EKF performance.

Applying this line of reasoning to the current problem results in

$$
\mathbf{P}^{-1}\left(t_{d}^{+}\right)=\left[\begin{array}{c|c}
\mathbf{P}_{\mathbf{r r}} & \mathbf{P}_{\mathbf{r v}} \\
\hline \mathbf{P}_{\mathbf{v r}} & \mathbf{P}_{\mathbf{v v}}
\end{array}\right]^{-1} \geq\left[\begin{array}{c|c}
\boldsymbol{\Theta}^{T} \mathbf{R}^{-1} \boldsymbol{\Theta} & \mathbf{0} \\
\hline \mathbf{0} & \mathbf{0}
\end{array}\right]
$$

where $\boldsymbol{\Theta}$ is defined in Eq. (2.26). Substituting the matrix identity for the inverse of a partitioned matrix [25] (for $\left.\mathbf{P}^{-1}\left(t_{d}^{+}\right)\right)$into Eq. (4.38) and manipulating the result, the following relationship is obtained:

$$
\mathbf{P}_{\mathbf{r r}}-\mathbf{P}_{\mathbf{r v}} \mathbf{P}_{\mathbf{v v}}^{-1} \mathbf{P}_{\mathbf{v r}} \leq\left(\Theta^{T} \mathbf{R}^{-1} \boldsymbol{\Theta}\right)^{-1}
$$

At time $t_{0}$ the matrices $\mathbf{P}_{\mathbf{r v}}$ and $\mathbf{P}_{\mathbf{v r}}$ are initialized to be zero and Eq. (4.39) offers the bound:

$$
\mathbf{P}_{\mathbf{r r}} \leq\left(\boldsymbol{\Theta}^{T} \mathbf{R}^{-1} \boldsymbol{\Theta}\right)^{-1}
$$

However, do to the system dynamics, correlations between position and velocity build-up and these matrices become nonzero. One method to incorporate these correlations while maintaining the direct form of the bound presented in Eq. (4.40) is

$$
\mathbf{P}_{\mathbf{r r}} \leq \kappa\left(\boldsymbol{\Theta}^{T} \mathbf{R}^{-1} \boldsymbol{\Theta}\right)^{-1}
$$

where $\kappa$ is a scaling factor defined by some function of the correlation coefficients. 
Recalling Eq. (4.7), the Gram form of the LTI observability matrix, $\mathcal{O}^{T} \mathcal{O}$, can be written as

$$
\mathcal{O}^{T} \mathcal{O}=\sum_{i=0}^{n-1}=\left(\mathbf{H F}^{i}\right)^{T}\left(\mathbf{H F}^{i}\right)=\left[\begin{array}{l|l}
\boldsymbol{\Gamma} & \mathbf{0} \\
\hline \mathbf{0} & \boldsymbol{\Gamma}
\end{array}\right]
$$

where

$$
\boldsymbol{\Gamma}:=\sum_{i=0}^{(n-2) / 2}\left(\boldsymbol{\Theta G}^{i}\right)^{T}\left(\boldsymbol{\Theta} \mathbf{G}^{i}\right) \approx \boldsymbol{\Theta}^{T} \boldsymbol{\Theta}
$$

Since $\mathbf{R}=\sigma_{\rho}^{2} \mathbf{I}$ implies $\Theta^{T} \mathbf{R}^{-1} \Theta \approx \boldsymbol{\Gamma} / \sigma_{\rho}^{2}$, it turns out that (loosely speaking) the observability matrix offers the following "upper bound" on the EKF position error covariance:

$$
\mathbf{P}_{\mathbf{r r}}\left(t_{d}^{+}\right) \leq \kappa \sigma_{\rho}^{2} \boldsymbol{\Gamma}^{-1}\left(t_{d}^{-}\right)
$$

Although further analysis would be required to determine $\kappa$ explicitly, for comparison sake, $\kappa=1$ is considered. The results are illustrated in Figure 4.21, where the $\sigma_{\rho}^{2} \boldsymbol{\Gamma}^{-1}$ "upper bound" is represented in gray and green for Case 2.0 and 2.1, respectively. Note that the addition of relative range measurements in Case 2.1 decreases the "upper bound" on the position error covariances in a manner that is consistent with the increase in observability described in Section 4.2.2. However, unlike the determinant observability measure illustrated in Figure 4.12, which generalizes the observability to the entire state, the metric presented in Eq. (4.44) offers insight into the observability of the individual components of the position. As discussed in Section 4.2.2 in terms of the resulting navigation performance, the $z$ component is the least 

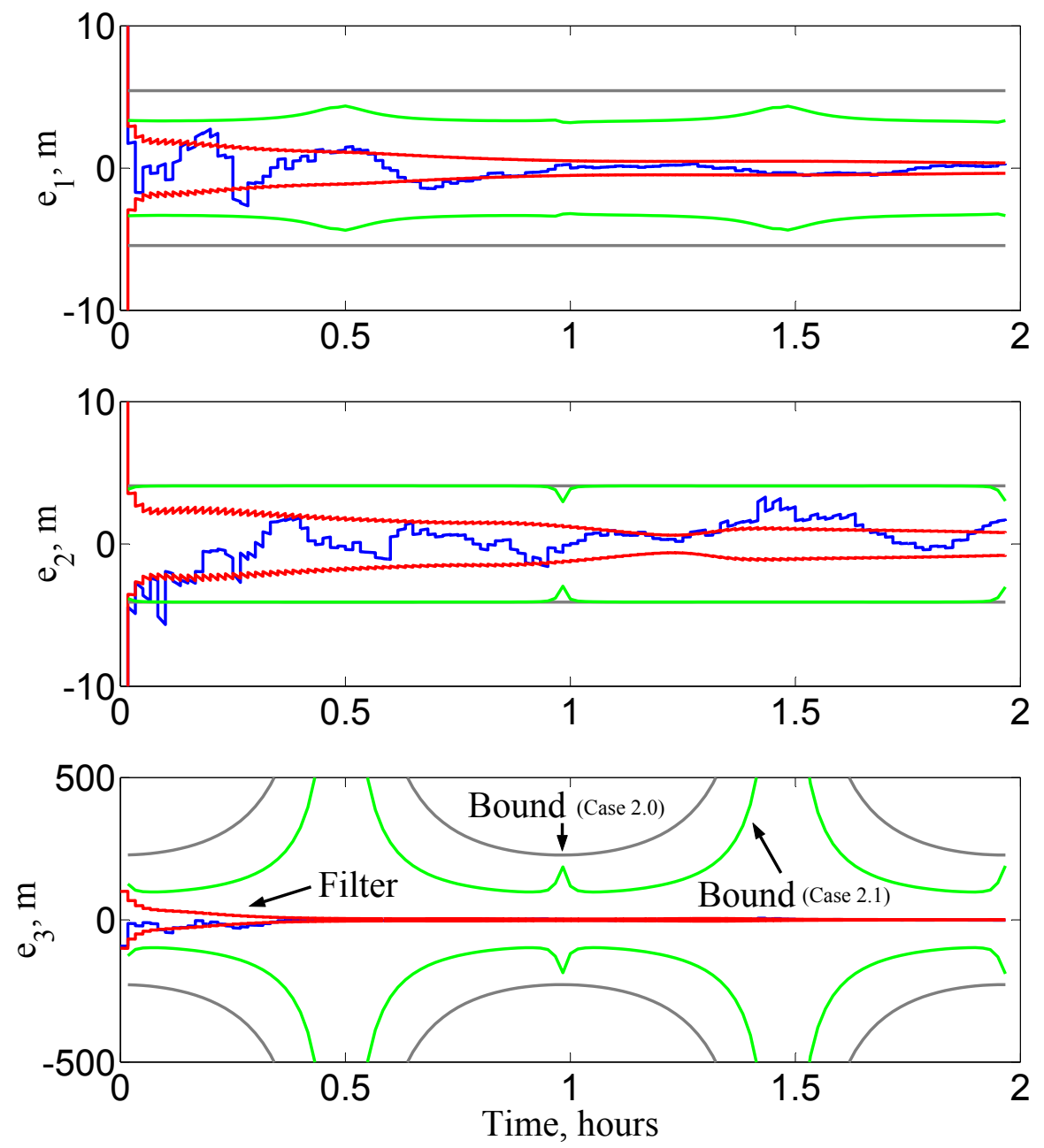

Figure 4.21: Time history of the position $\sigma_{\rho}^{2} \boldsymbol{\Gamma}^{-1}$ "upper bound" for Case 2.1. observable and receive the largest increase in observability due to the relative range measurements, followed then by the $x$ component. 
The bulges in the $\sigma_{\rho}^{2} \boldsymbol{\Gamma}^{-1}$ "upper bound" in Figure 4.21 for Case 2.1 correspond to the scenarios discussed in Section 4.2.2 and illustrated geometrically in Figure 4.8. Specifically, the slight bulge in the $x$ component near 0.5 and 1.5 hours corresponds to $X_{I^{-}} Y_{I}$ plane crossing. Due to the nodal crossing at these points the relative range measurements have reduced components in the $X_{I}$ direction. Likewise the $y$ component experiences a slight decrease near 0,1 , and 2 hours because at these points the relative range measurements are mostly in the $Y_{I}$ direction. Finally, since the $z$ component is dominated by the poor inertial observability in this direction, large spikes occur near 0.5 and 1.5 hours due the $X_{I}-Y_{I}$ plane crossing (recall the inertial tracking stations lie in the $X_{I}-Y_{I}$ plane). However, the smaller spikes near 0, 1, and 2 hours occur because at these points the relative range measurements have reduced components in the $Z_{I}$ direction.

Finally, although this analysis results in a fairly conservative bound, it does have the advantage that it is completely analytic. As demonstrated in Sections 4.3.2 and 4.3.3, the Gramian-based metrics can be used to forecast a covariance level more indicative of the actual navigation performance, but computing the observability Gramian requires the integration of the state transition matrix via Eq. (3.16).

\subsubsection{Cramér-Rao Covariance Bound}

Perhaps the most common bound on the state error covariance of an

unbiased estimate is the Cramér-Rao inequality [53], which states that for 
$\mathcal{J}\left(t_{0}\right)=\mathbf{P}^{-1}\left(t_{0}\right)$

$$
\mathbf{P}(t) \geq \boldsymbol{J}^{-1}(t)
$$

where $\mathcal{J}$ is the Fisher information matrix. Thus, the inverse of the Fisher information matrix provides a lower bound for the best achievable state estimate error covariance.

Recall the Information Form of the Kalman filter, which by manipulating Eqs. (3.32) and (3.35), instead propagates the inverse of the state error covariance using Eq. (4.36). Although algebraically equivalent to the standard propagation procedure, this alternate form of the Kalman filter has the numerical advantage of being able to accommodate the initial assumption of no a priori information, since although $\mathbf{P}\left(t_{0}\right)=\infty$ is not numerically realizable, $\mathbf{P}^{-1}\left(t_{0}\right)=\mathbf{0}$ is finite.

With this in mind, Taylor [51] asserts that for the continuous-discrete EKF (described in Chapter 3), "the covariance propagation equations linearized about the true unknown trajectory provide the Cramér-Rao lower bound to the estimation error covariance matrix." He demonstrates this by obtaining the Fisher information matrix in recursive form as,

$$
\mathcal{J}\left(t_{d}\right)=\boldsymbol{\Phi}^{-T}\left(t_{d}, t_{d-1}\right) \mathfrak{J}\left(t_{d-1}\right) \boldsymbol{\Phi}^{-1}\left(t_{d}, t_{d-1}\right)+\mathbf{H}^{T}\left(t_{d}\right) \mathbf{R}^{-1}\left(t_{d}\right) \mathbf{H}\left(t_{d}\right)
$$

which given the a priori information that $\mathcal{J}\left(t_{0}\right)=\mathbf{P}^{-1}\left(t_{0}\right)$ and recalling Eq. (3.26) in the absence of process noise $\left(\mathbf{Q}\left(t_{d}\right)=\mathbf{0}\right)$, is identical to Eq. (4.36). 
Alternatively, Eq. (4.46) is equivalently written in summation form as,

$$
\begin{aligned}
\mathcal{J}\left(t_{d}\right)= & \boldsymbol{\Phi}^{-T}\left(t_{d}, t_{0}\right) \mathbf{P}^{-1}\left(t_{0}\right) \boldsymbol{\Phi}^{-1}\left(t_{d}, t_{0}\right) \\
& +\sum_{k=1}^{d} \boldsymbol{\Phi}^{-T}\left(t_{d}, t_{k}\right) \mathbf{H}^{T}\left(t_{k}\right) \mathbf{R}^{-1}\left(t_{k}\right) \mathbf{H}\left(t_{k}\right) \boldsymbol{\Phi}^{-1}\left(t_{d}, t_{k}\right)
\end{aligned}
$$

which implies that $\mathcal{J}\left(t_{0}\right)=\mathbf{P}^{-1}\left(t_{0}\right)$. Note however, that when this a priori information is disregarded $\left(\mathbf{P}^{-1}\left(t_{0}\right)=\mathbf{0}\right)$, Eq. (4.47) reduces to the more familiar form presented in Maybeck [39] and then describes the certainty of the state estimate due only to the measurement data.

Recalling Section 4.1.3, their similar forms would indicate that there is natural relationship between the Fisher information matrix and the observability Gramian. By considering measurement noise such that $\mathbf{R}\left(t_{d}\right)=\sigma_{\rho}^{2} \mathbf{I}$ and comparing Eqs. (4.15) and (4.46), it can be shown that this is in fact true. To illustrate this, first define the matrix $\mathcal{L}\left(t_{d}\right)$ as the rearrangement of Eq. (4.15) resulting from left multiplying by $\boldsymbol{\Phi}^{-1}\left(t_{d}, t_{1}\right)$, right multiplying by $\boldsymbol{\Phi}^{-1}\left(t_{d}, t_{1}\right)$, and dividing by $\sigma_{\rho}^{2}$ such that

$$
\begin{aligned}
\mathcal{L}\left(t_{d}\right) & :=\boldsymbol{\Phi}^{-T}\left(t_{d}, t_{1}\right) \frac{\mathcal{M}\left(t_{d}\right)}{\sigma_{\rho}^{2}} \boldsymbol{\Phi}^{-1}\left(t_{d}, t_{1}\right) \\
& =\boldsymbol{\Phi}^{-T}\left(t_{d}, t_{1}\right) \frac{\mathcal{M}\left(t_{d-1}\right)}{\sigma_{\rho}^{2}} \boldsymbol{\Phi}^{-T}\left(t_{d}, t_{1}\right)+\frac{\mathbf{H}^{T}\left(t_{d}\right) \mathbf{H}\left(t_{d}\right)}{\sigma_{\rho}^{2}}
\end{aligned}
$$

Next, recognizing that the first term of the second line can be rewritten in terms of $\mathcal{L}\left(t_{d-1}\right)$ using the definition on the first line, Eq. (4.48) results in

$$
\mathcal{L}\left(t_{d}\right)=\boldsymbol{\Phi}^{-T}\left(t_{d}, t_{d-1}\right) \mathcal{L}\left(t_{d-1}\right) \boldsymbol{\Phi}^{-1}\left(t_{d}, t_{d-1}\right)+\frac{\mathbf{H}^{T}\left(t_{d}\right) \mathbf{H}\left(t_{d}\right)}{\sigma_{\rho}^{2}}
$$

which is identical to the form of Eq. (4.46) when $\mathbf{R}\left(t_{d}\right)=\sigma_{\rho}^{2} \mathbf{I}$. Consequently it is tempting to conclude that the solution to Eq. (4.49) is $\mathcal{L}\left(t_{d}\right)=\mathfrak{J}\left(t_{d}\right)$. 
However, while this is true in the absence of a priori information, in order to satisfy the initial conditions $\mathcal{J}\left(t_{0}\right)=\mathbf{P}^{-1}\left(t_{0}\right)$ and $\mathcal{M}\left(t_{0}\right)=\mathbf{0}$ the general solution is actually,

$$
\mathcal{L}\left(t_{d}\right)=\mathcal{J}\left(t_{d}\right)-\boldsymbol{\Phi}^{-T}\left(t_{d}, t_{0}\right) \mathbf{P}^{-1}\left(t_{0}\right) \boldsymbol{\Phi}^{-1}\left(t_{d}, t_{0}\right)
$$

Equating Eq. (4.50) with the definition of $\mathcal{L}\left(t_{d}\right)$ in Eq. (4.48) results in the following general relationship between the Fisher information matrix and the observability Gramian:

$$
\mathcal{J}\left(t_{d}\right)=\boldsymbol{\Phi}^{-T}\left(t_{d}, t_{0}\right) \mathbf{P}^{-1}\left(t_{0}\right) \boldsymbol{\Phi}^{-1}\left(t_{d}, t_{0}\right)+\boldsymbol{\Phi}^{-T}\left(t_{d}, t_{1}\right) \frac{\mathcal{M}\left(t_{d}\right)}{\sigma_{\rho}^{2}} \boldsymbol{\Phi}^{-1}\left(t_{d}, t_{1}\right)
$$

Recall that for the idealized problems examined in this dissertation, the environment (truth) and filter models are identical and consequently $\mathbf{Q}\left(t_{d}\right)=$ 0. Also, note that if the state errors are small enough, linearization about the estimated trajectory will be essentially the same as linearization about the true trajectory. Furthermore, since the errors are sufficiently reduced after only a few updates, the a posteriori state error covariance of the filter is nearly the same as the inverse of the Fisher information matrix. Consequently, as seen in green in Figures 4.22 and 4.23, Eq. (4.51) yields a tight lower bound on the state error covariances of Case 2.1, previously presented in Figures 4.13 and 4.14 .

Alternatively, suppose it is desirable to determine the best achievable state error covariance without a priori information. In such a case, $\mathbf{P}^{-1}\left(t_{0}\right)=$ 

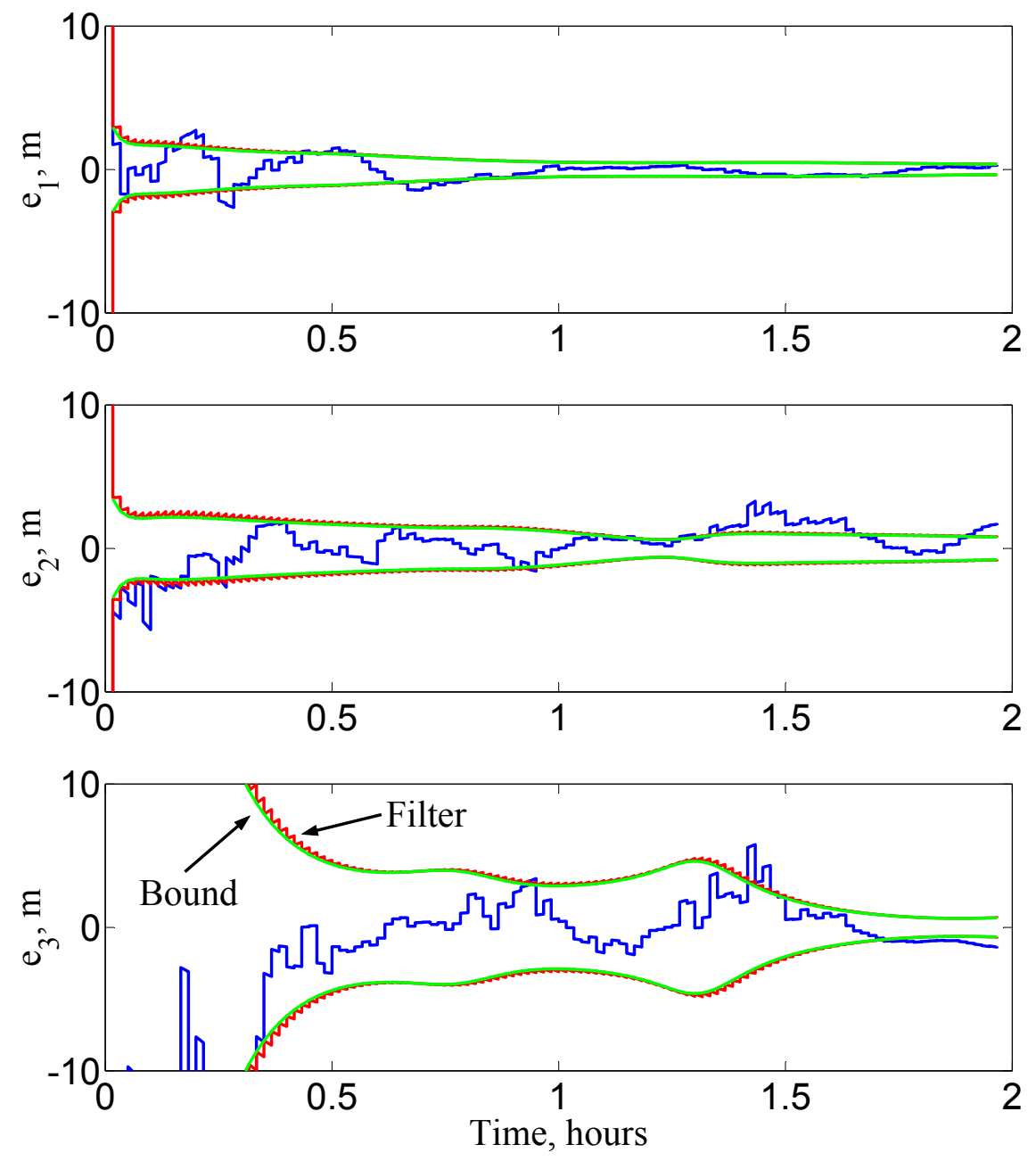

Figure 4.22: Time history of the position $\mathfrak{J}^{-1}$ lower bound for Case 2.1.

$\mathbf{0}$ and Eq. (4.51) defines the new Fisher information matrix,

$$
\mathcal{J}_{\infty}\left(t_{d}\right):=\boldsymbol{\Phi}^{-T}\left(t_{d}, t_{1}\right) \frac{\mathcal{M}\left(t_{d}\right)}{\sigma_{\rho}^{2}} \boldsymbol{\Phi}^{-1}\left(t_{d}, t_{1}\right)
$$



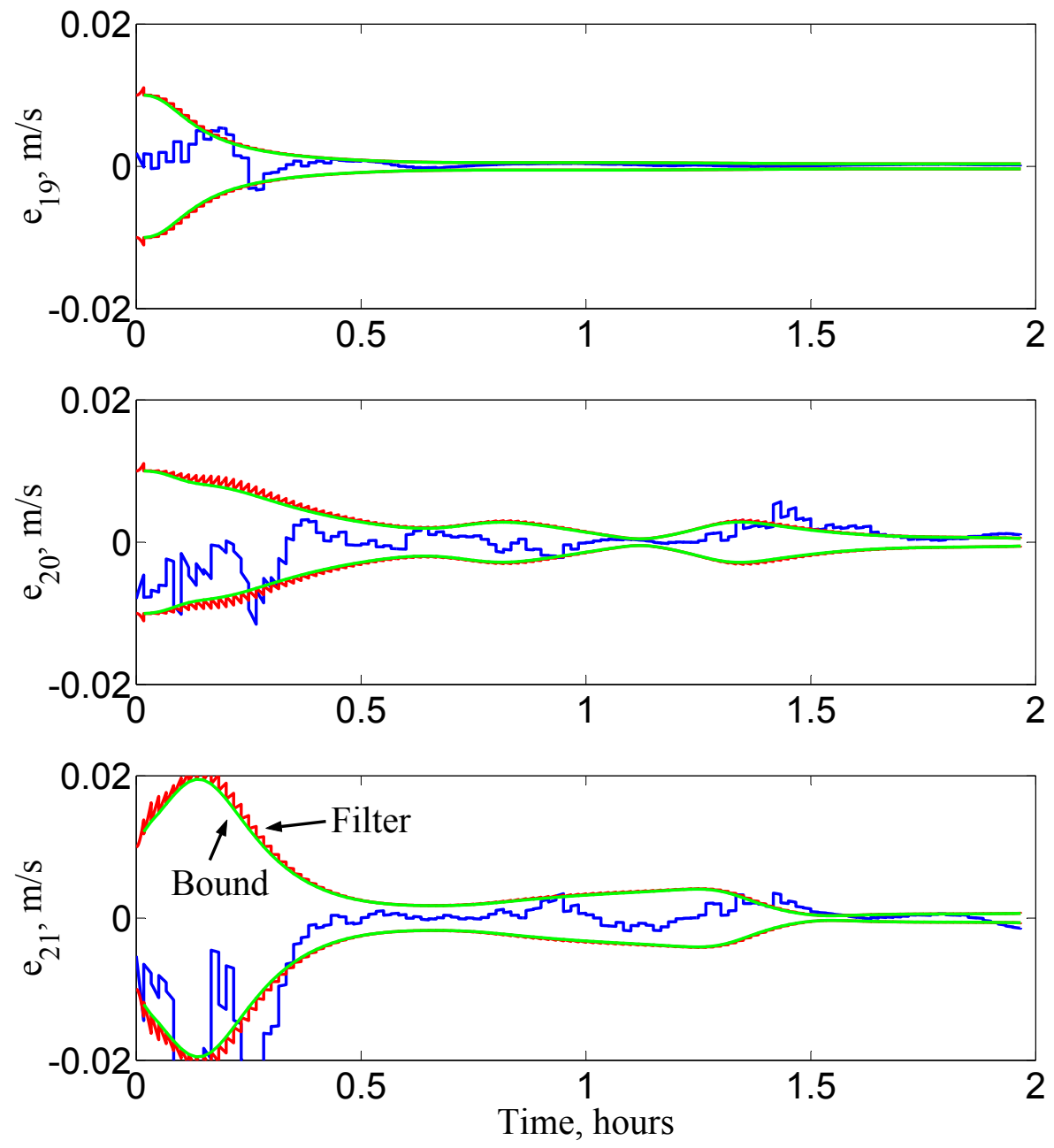

Figure 4.23: Time history of the velocity $\mathfrak{J}^{-1}$ lower bound for Case 2.1.

While this Fisher information matrix provides a lower bound on the information filter state error covariance (provided it is initialized with $\mathbf{P}^{-1}\left(t_{0}\right)=\mathbf{0}$ ), it 
does not provided a lower bound on the state error covariance of the standard Kalman filter, since it is not numerically possible to initialize the filter with $\mathbf{P}\left(t_{0}\right)=\infty$. Consequently, since the filter is starting with more information than is used to compute the Fisher information matrix, logically the filter can at times perform better than Eq. (4.52) might indicate.

In particular, recall that for the idealized problems examined in this research, the a posteriori state error covariance of the filter is nearly the same as the inverse of the Fisher information matrix presented in Eq. (4.51). Next, consider that since the first term in Eq. (4.51) is always positive definite for $\mathbf{P}^{-1}\left(t_{0}\right)>\mathbf{0}$, the general Fisher information matrix is always bounded such that, $\mathfrak{J}\left(t_{d}\right)>\mathcal{J}_{\infty}\left(t_{d}\right)$. Combining these results, loosely speaking Eq. (4.52) offers an "upper bound" on the a posteriori state error covariance, since $\mathbf{P}\left(t_{d}^{+}\right) \approx \boldsymbol{J}^{-1}\left(t_{d}\right)<\boldsymbol{J}_{\infty}^{-1}\left(t_{d}\right)$. This result is demonstrated in green in Figures 4.24 and 4.25 for Case 2.1.

For the sake of comparison with the DOP metrics discussed in Section 4.3.3, as well to provide a more direct relationship between the navigation performance and the system observability, consider instead the metric $\sigma_{\rho}^{2} \mathcal{M}^{-1}\left(t_{d}\right)$. In order for this metric to continue the role as an "upper bound" requires that $\mathcal{J}_{\infty}^{-1}\left(t_{d}\right) \leq \sigma_{\rho}^{2} \mathcal{M}^{-1}\left(t_{d}\right)$, which according to Eq. (4.52) then implies that

$$
\boldsymbol{\Phi}^{-T}\left(t_{d}, t_{1}\right) \mathcal{M}\left(t_{d}\right) \boldsymbol{\Phi}^{-1}\left(t_{d}, t_{1}\right) \geq \mathcal{M}\left(t_{d}\right)
$$

In general there is no guarantee the inequality of Eq. (4.53) will hold, and the equality requires that $\boldsymbol{\Phi}\left(t_{d}, t_{1}\right)=\mathbf{I}$; which, since $\dot{\boldsymbol{\Phi}}=\mathbf{F} \boldsymbol{\Phi}$ with initial 

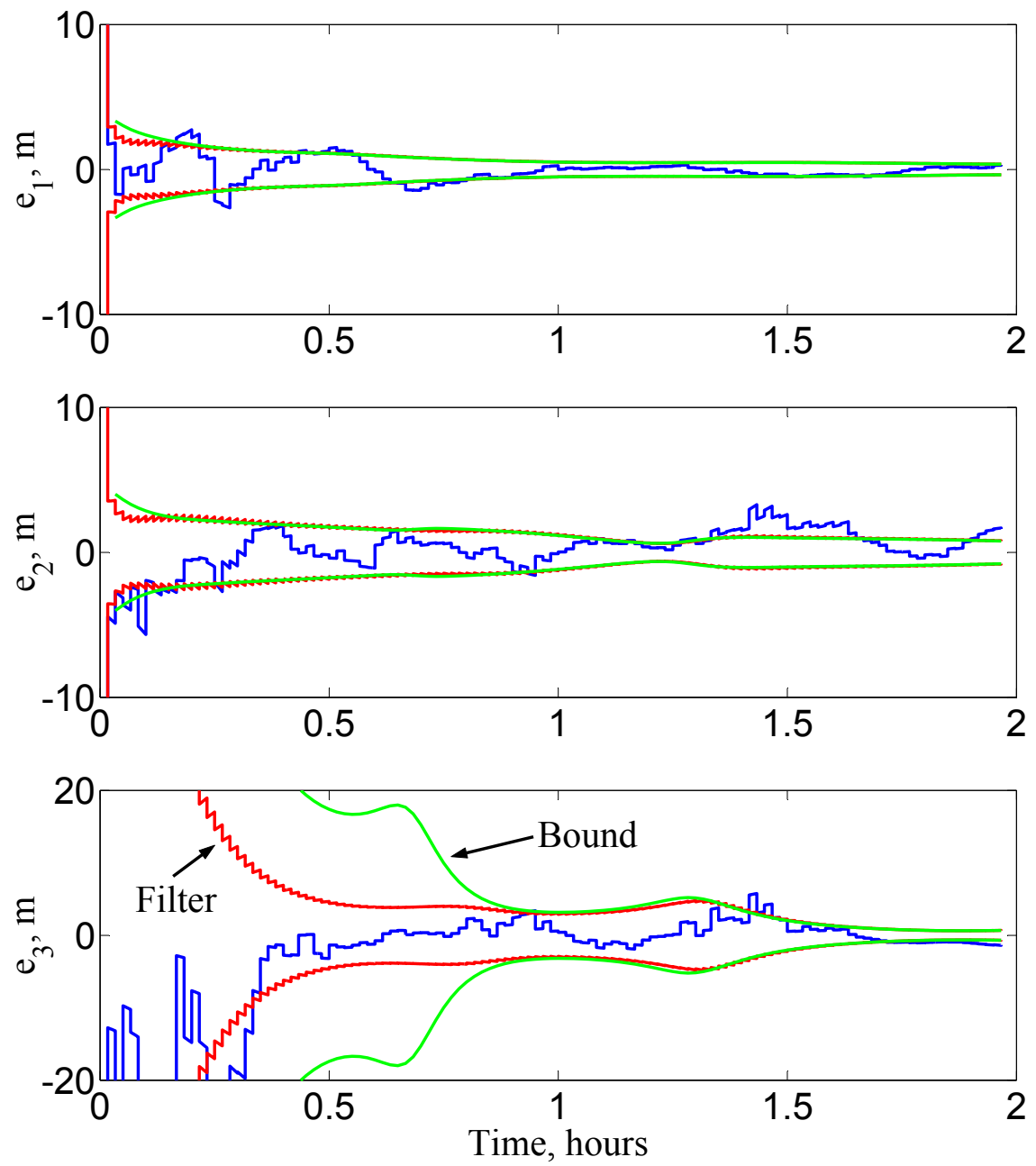

Figure 4.24: Time history of the position $\boldsymbol{J}_{\infty}^{-1}$ "upper bound" for Case 2.1.

condition $\mathbf{\Phi}\left(t_{d-1}, t_{d-1}\right)=\mathbf{I}$, then restricts the state to be constant (i.e. $\mathbf{F}=\mathbf{0}$ ). This restriction is obviously not reasonable for typical orbital applications and 

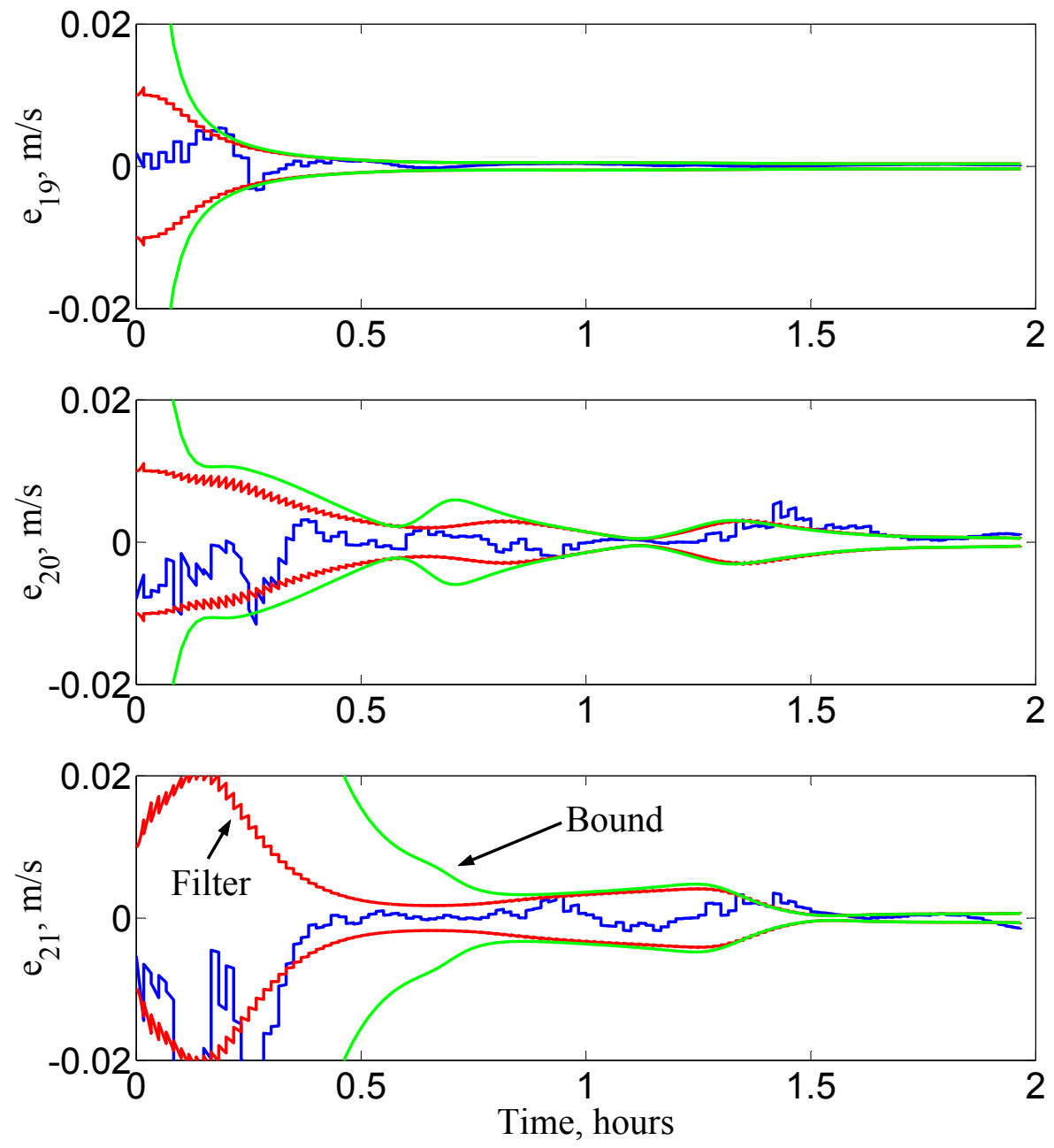

Figure 4.25: Time history of the velocity $\mathfrak{J}_{\infty}^{-1}$ "upper bound" for Case 2.1.

consequently this metric cannot guarantee a bounding relationship with the state error covariance. 

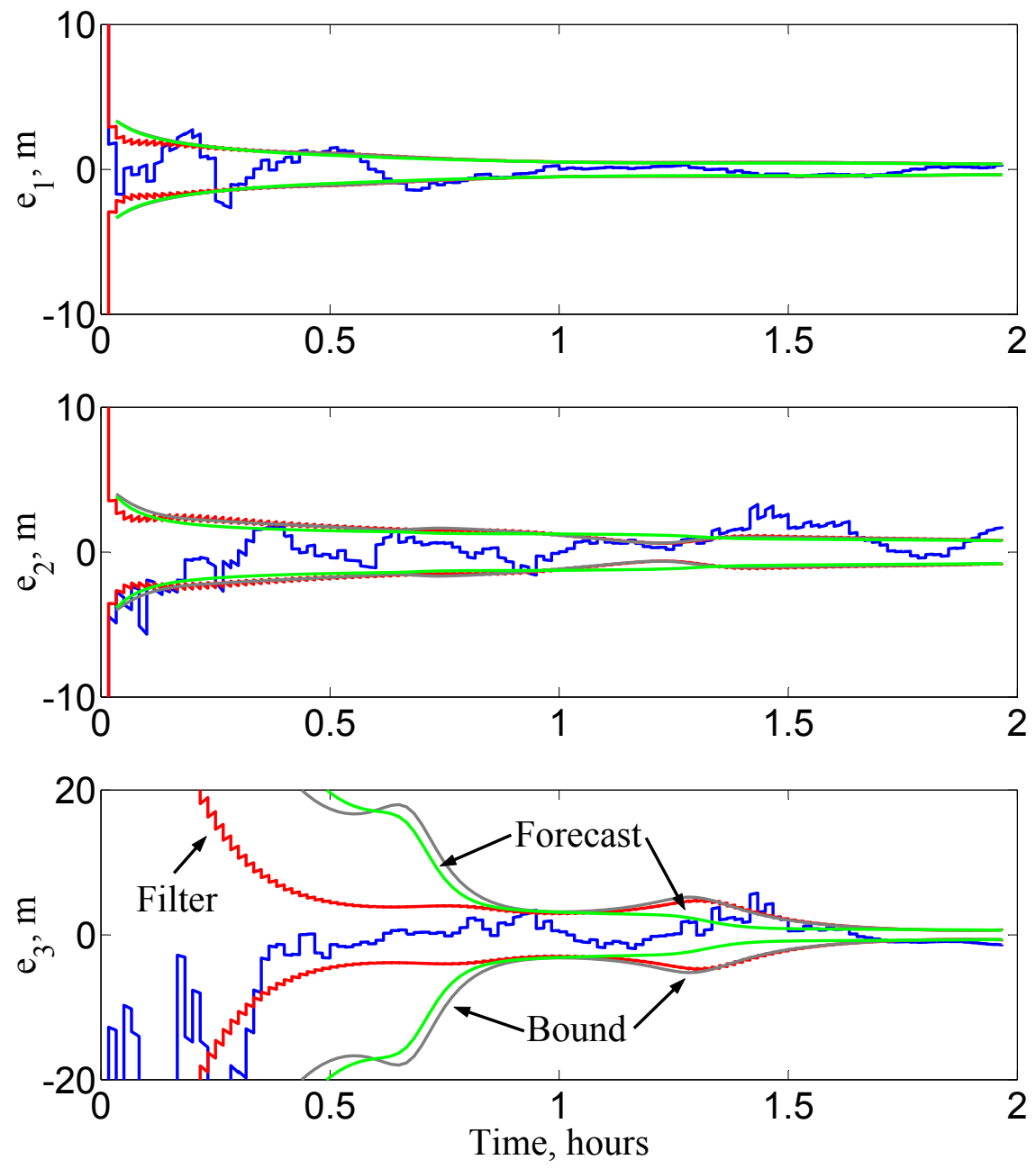

Figure 4.26: Time history of the position $\sigma_{\rho}^{2} \mathcal{N}^{-1}$ forecast for Case 2.1.

This is further demonstrated in Figures 4.26 and 4.27 for Case 2.1. In these figures, the $\sigma_{\rho}^{2} \mathcal{M}^{-1}$ metric is represented in green, while the previous 

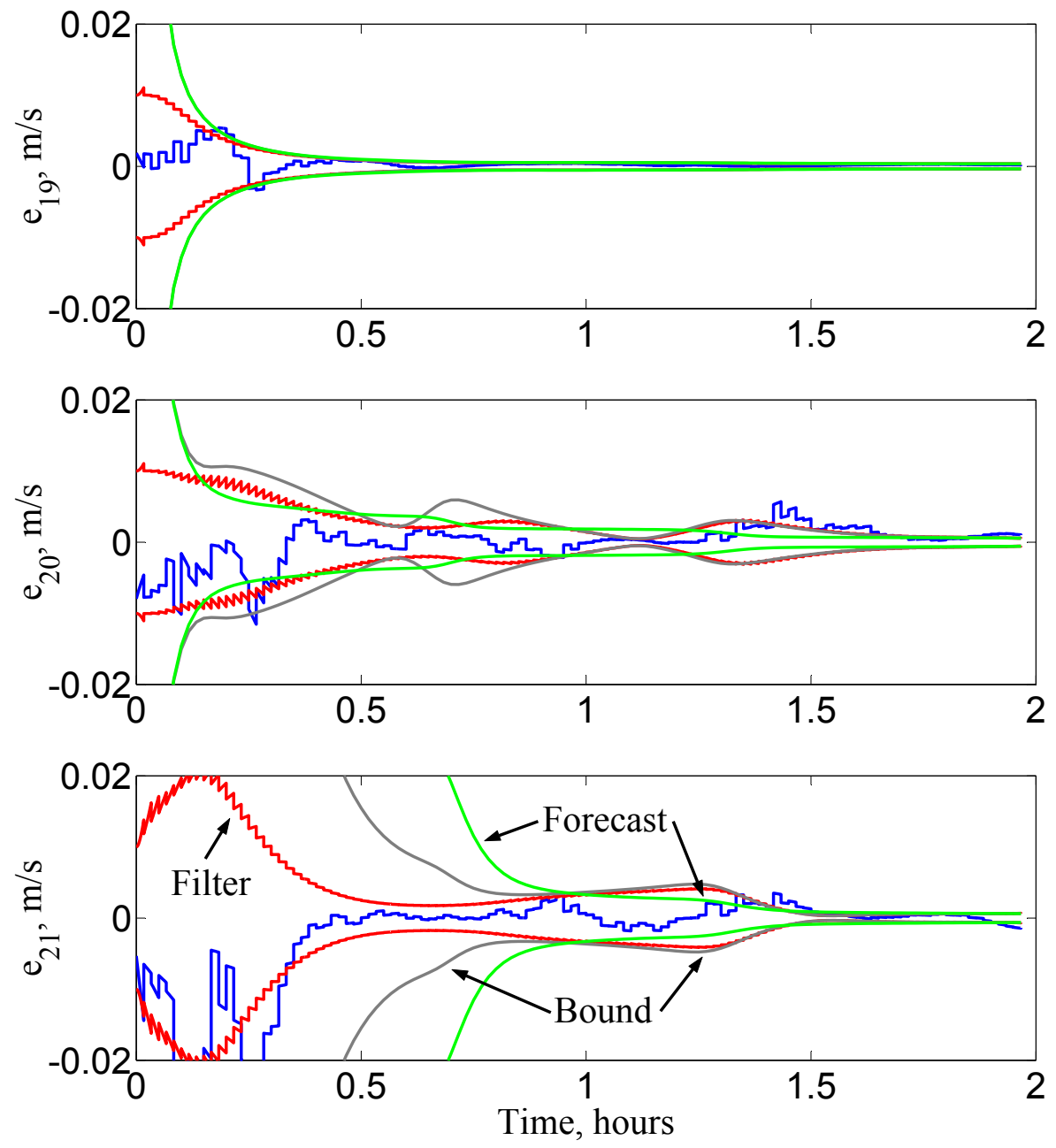

Figure 4.27: Time history of the velocity $\sigma_{\rho}^{2} \mathcal{M}^{-1}$ forecast for Case 2.1.

$\mathfrak{J}_{\infty}^{-1}$ "upper bound" of Eq. (4.52) has also been included in gray for ease of comparison. Finally, although this metric does not offers an explicit bound, 
particularly in the $z$-position and $y / z$-velocities, it still offers a rough "forecast" of the state error covariance based on the measurement data.

\subsubsection{DOP-Based Covariance Forecast}

The NASA Space Communication Architecture Working Group (SCAWG) Navigation Team has proposed a set of metrics for navigation analysis [46]. In particular the SCAWG team discusses how the inverse of the observability Gramian can be used to indicate how the least-squares solution maps

projections of the measurement data $\left(\boldsymbol{\Xi}^{T} \boldsymbol{\zeta}\right)$ into the solution space (see Eq. (4.5)); and more specifically how the geometry of the measurement batch acts to dilute (or concentrate) this mapping. One example of this is the geometric dilution of precision (DOP) metric that is commonly used in global positioning system (GPS) receivers $[43,46,56]$. Expanding upon the standard geometric DOP the SCAWG team proposes instead the so-called generalized geometric DOP, which is equal to the square root of the 2-norm of the inverse of the observability Gramian.

Similar to the determinant observability measure, the DOP-type metrics results in a scalar measure of observability generalized for the entire state vector. Alternatively, the SCAWG team shows that, in general, the inverse of the observability Gramian can instead be mapped into any desired variable. For example, to examine the observability of the alternate state $\mathbf{s}$ would instead require computing the generalized DOP of the following metric,

$$
\mathcal{D}_{\mathbf{s}}\left(t_{d}\right)=\mathbf{S}\left(t_{d}\right) \mathcal{M}^{-1}\left(t_{d}\right) \mathbf{S}^{T}\left(t_{d}\right)
$$


where $\mathbf{s}=\mathbf{s}(\mathbf{x})$ and

$$
\mathbf{S}\left(t_{d}\right):=\left.\frac{\partial \mathbf{s}}{\partial \mathbf{x}}\right|_{\mathbf{x}=\mathbf{x}\left(t_{d}\right)}
$$

In this way the observability of position and velocity can be examined separately by considering $\mathbf{s}=\mathbf{r}$ and $\mathbf{s}=\mathbf{v}$, respectively, where

$$
\mathbf{r}:=\left[\begin{array}{c}
\mathbf{r}_{1} \\
\vdots \\
\mathbf{r}_{p}
\end{array}\right] \text { and } \mathbf{v}:=\left[\begin{array}{c}
\mathbf{v}_{1} \\
\vdots \\
\mathbf{v}_{p}
\end{array}\right]
$$

Another contribution of the SCAWG team is the development of the conditions under which these generalized DOP metrics can provided a rough estimate of the navigation accuracy. Coincidentally these condition are consistent with the circumstances considered in Section 4.3.2 concerning the $\mathcal{J}$-based bounds. Namely, $(i)$ the measurements are of uniform type and accuracy $\left(\mathbf{R}\left(t_{d}\right)=\sigma_{\rho}^{2} \mathbf{I}\right),(i i)$ there are no uncertain inputs to the system dynamics $\left(\mathbf{Q}\left(t_{d}\right)=\mathbf{0}\right),($ iii $)$ there is no a priori information $\left(\mathbf{P}^{-1}\left(t_{0}\right)=\mathbf{0}\right),(i v)$ the filter models accurately reflect the environment, and $(v)$ the state used during the linearization is close enough to the actual state that neglected higher order terms do not become significant. With these conditions met, $\mathcal{D}_{\text {s }}$ can be multiplied by $\sigma_{\rho}^{2}$ and the generalized position and velocity DOP metrics can be computed to obtain an estimate of the position and velocity error covariances such that $\mathbf{P}_{\mathbf{r r}} \sim \sigma_{\mathbf{r}}^{2} \mathbf{I}$ and $\mathbf{P}_{\mathbf{v v}} \sim \sigma_{\mathbf{v}}^{2} \mathbf{I}$ where,

$$
\sigma_{\mathbf{r}}:=\sigma_{\rho} \sqrt{\left\|\mathcal{D}_{\mathbf{r}}\right\|} \text { and } \quad \sigma_{\mathbf{v}}:=\sigma_{\rho} \sqrt{\left\|\mathcal{D}_{\mathbf{v}}\right\|}
$$

The resulting DOP-based covariance forecast is demonstrated in green in Figures 4.28 and 4.29 for Case 2.1. Notice that since the metric is generalized 

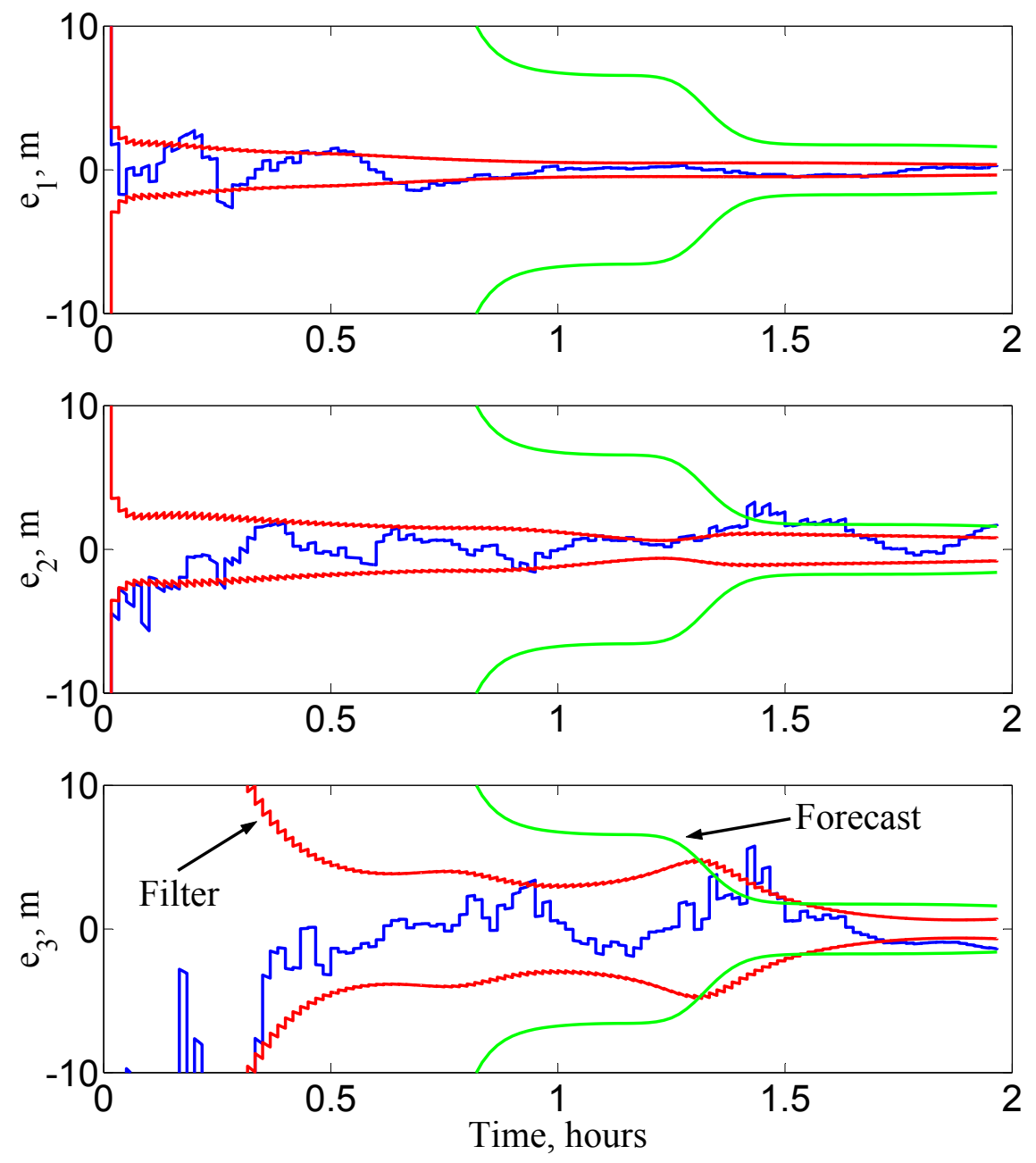

Figure 4.28: Time history of the position $\sigma_{\rho}^{2}\left\|\mathcal{D}_{\mathbf{r}}\right\| \mathbf{I}$ forecast for Case 2.1.

to $\mathbf{r}$ and $\mathbf{v}$ (and not individual state components), the resulting forecast is the same for all $x, y$, and $z$ components. In particular, since the $z$ components 

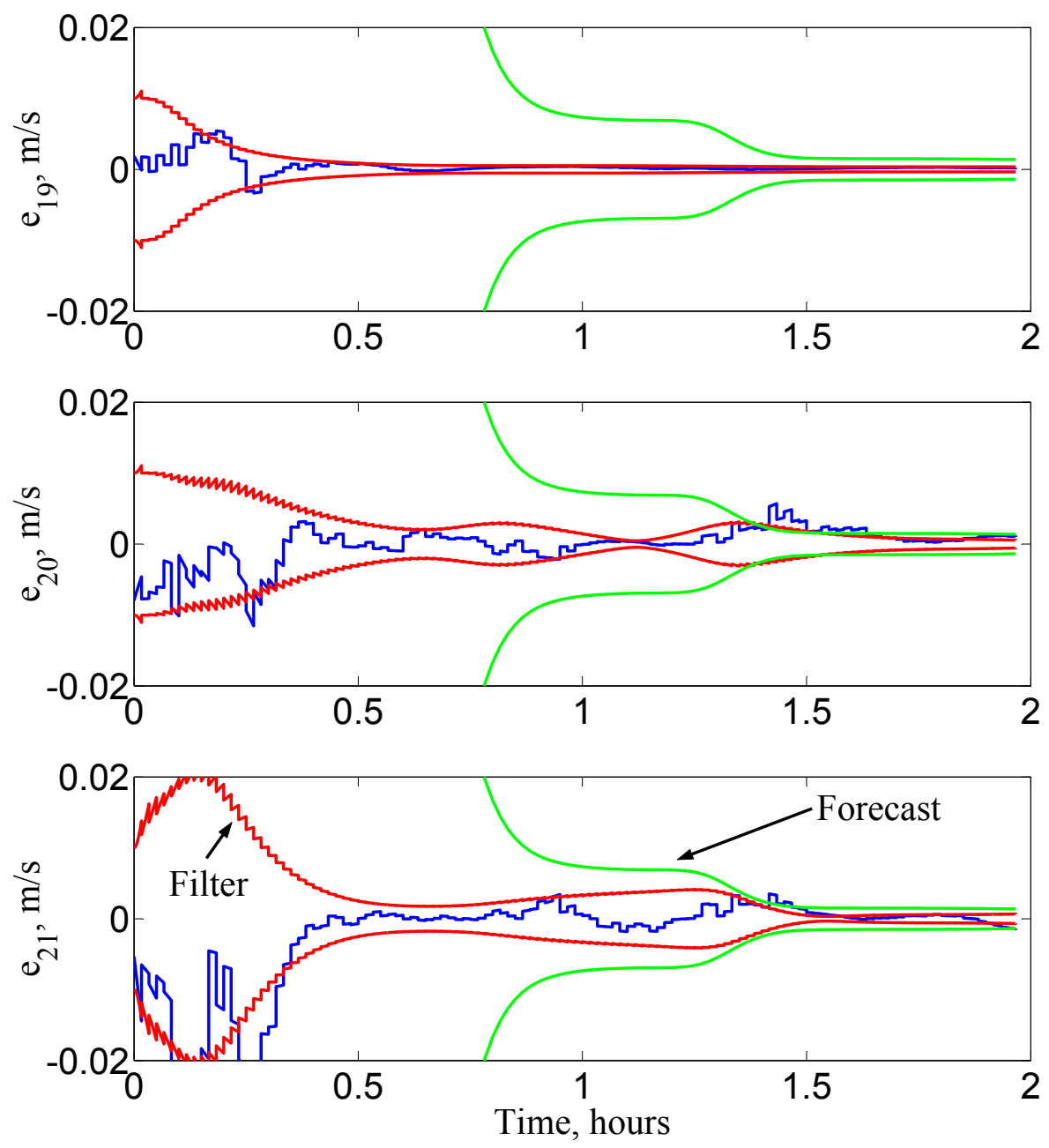

Figure 4.29: Time history of the velocity $\sigma_{\rho}^{2}\left\|\mathcal{D}_{\mathbf{v}}\right\| \mathbf{I}$ forecast for Case 2.1.

are the least observable, they tend to dominate the forecast resulting in a very conservative estimates for the $x$ and $y$ components. Consequently a more 
interesting metric is obtained by choosing $\mathbf{s}$ to be a scalar such that the state is considered component by component (i.e. $s=x_{i}$ for $i=1, \ldots, n$ ). Doing so results in a scalar $\mathcal{D}_{s}$ equal to the diagonal elements of $\mathcal{M}^{-1}$ corresponding to the chosen $s$. Thus the covariance forecast for each state element is then simply the corresponding diagonal element of $\sigma_{\rho}^{2} \mathcal{M}^{-1}$. Since only the diagonal elements are of interested, this particular DOP metric is then equivalent to the $\sigma_{\rho}^{2} \mathcal{M}^{-1}$ metric proposed in Section 4.3.2 and demonstrated in Figures 4.26 and 4.27 .

\subsection{Summary of Observability Simulation Results}

This chapter used various observability measures to examine the relationship between observer geometry and the navigation performance of the EKF for several different lunar mission cases. Using the LTI observability condition defined in Definition 4.1.1, possible reductions in observability where examined when using inertial range measurements due to the following geometric anomalies:

- 1 spacecraft and the 2 or more stations tracking it are collinear

- 1 spacecraft and the 3 or more stations tracking it are coplanar

These results were then expanded to include the use of relative range measurements and presented the following additional geometric anomalies:

- 2 spacecraft and the station(s) tracking each are collinear 
- the line connecting 2 spacecraft is parallel to the line connecting the 2 stations tracking each spacecraft (see Figure 4.9)

- 3 spacecraft are collinear

- 4 spacecraft are coplanar

The last anomaly was the result of examining the LTI observability measure in an attempt to determine the optimal constant formation geometry based on the relative range measurements. Figures 4.15 and 4.18 illustrated the ideal formation for three spacecraft in two dimensions and four spacecraft in three dimensions, respectively.

The next step in the analysis was to explicitly relate the observability measure to the expected navigation performance. This was demonstrated through the use of the least-squares and the Cramér-Rao covariance bounds. The least-squares approach provided a conservative, yet analytic, relationship between the LTI observability and the expected navigation performance. The exact relationship between the Fisher information matrix (used in the CramérRao bound) and the observability Gramian was then derived in Eq. (4.51) to provided a more representative covariance forecast. Finally, using this relationship, the connection between the Cramér-Rao covariance bound and the DOP metric covariance forecast was described. 


\section{Chapter 5}

\section{Relative Range Measurement Fusion}

While investigating the observability of the spacecraft formation, an interesting phenomenon was noted. In the presence of large initial state estimate error covariance, fusing ${ }^{1}$ relative range measurements with inertial range measurements ${ }^{2}$ caused an otherwise convergent filter (using inertial range measurements only) to diverge. To illustrate the complications involved in fusing relative and inertial range measurements, the six-spacecraft circular polar orbit mission is reconsidered for two different state error covariance levels: moderate (Case 2) and severe (Case 3). The magnitudes of these levels of state error covariance are presented in Table 5.1.

After examining the complications associated with this type of measurement fusion, several robustness enhancement schemes are considered for fusing measurements in the presence of a severe level of state error covariance.

\footnotetext{
${ }^{1}$ Since by formulation of the EKF fuses all measurements into a unified measurement vector, practically speaking measurement fusion occurs between all measurements. However, for discussion purposes, the terms "fusing" and "fusion" are used to refer specifically to the combining of a set of inertial measurements with a set of relative measurements.

${ }^{2}$ Recall that the term "inertial range measurement" refers to a range measurement taken from an observer with a known ephemeris in the inertial frame; while the the term "relative range measurement" refers to a range measurement taken between spacecraft contained in the estimated state.
} 


\begin{tabular}{lcc} 
Table 5.1: & State Estimate Error Covariance Levels \\
\hline Level & Position $(\mathrm{km})$ & Velocity $(\mathrm{km} / \mathrm{s})$ \\
\hline Moderate & $\sigma_{\mathbf{r}} \sim 10^{-1}$ & $\sigma_{\mathbf{v}} \sim 10^{-5}$ \\
Severe & $\sigma_{\mathbf{r}} \sim 10^{+1}$ & $\sigma_{\mathbf{v}} \sim 10^{-3}$ \\
\hline \hline
\end{tabular}

These schemes, which range from some commonly used first-order numerical methods to second-order analytical methods, are:

(0) No Robustness Enhancement Scheme (EKF)

(I) First-Order Methods (EKF)
(A) Scalar Update Scheme
(B) Process Noise Scheme
(C) Measurement Noise Scheme
(D) Selective Measurement Scheme

(II) Second-Order Methods (GSF)

After reviewing the performance of the first-order methods, the algorithms of the second-order method are investigated to determine their potential contribution to solving the challenges of fusing inertial and relative range measurements. All first-order methods are based on the standard EKF equations, while the GSF is used as the basis for the second-order methods. 


\subsection{Fusion Complications}

Using the initial state error covariance described in Table 5.1, the following test results are obtained: $(i)$ the EKF converges for a moderate level of covariance when only inertial range measurements are used (Case 2.0), (ii) the EKF converges for a moderate level of covariance when fusing relative measurements (Case 2.1), (iii) the EKF converges for a severe level of covariance when only inertial measurements are used (Case 3.0), and (iv) the EKF $d i$ verges for a severe level of covariance when fusing relative measurements (Case 3.1). Table 5.2 summarizes these test cases and results.

Table 5.2: Fusion Simulation Test Cases

\begin{tabular}{cccc}
\hline \hline Case & Covariance Level & Measurement Profile & Filter Performance \\
\hline 2.0 & moderate & inertial only & converges \\
2.1 & moderate & relative fusion & converges \\
3.0 & severe & inertial only & converges \\
3.1 & severe & relative fusion & diverges \\
\hline \hline
\end{tabular}

The divergence of the navigation state error for Case 3.1 (without the use of a robustness enhancement scheme) is illustrated in Figures 5.1 and 5.2. The results show that the state error lies well outside the predicted state error covariance bounds. Also, according to the filter covariance, the navigation performance is comparable to that of Case 2. Based on the actual state errors this is not true. Although in practice the actual state errors are unknown, this filter failure can be detected be examining the residuals. However, the inconsistency between the actually errors and the filter predicted state error 

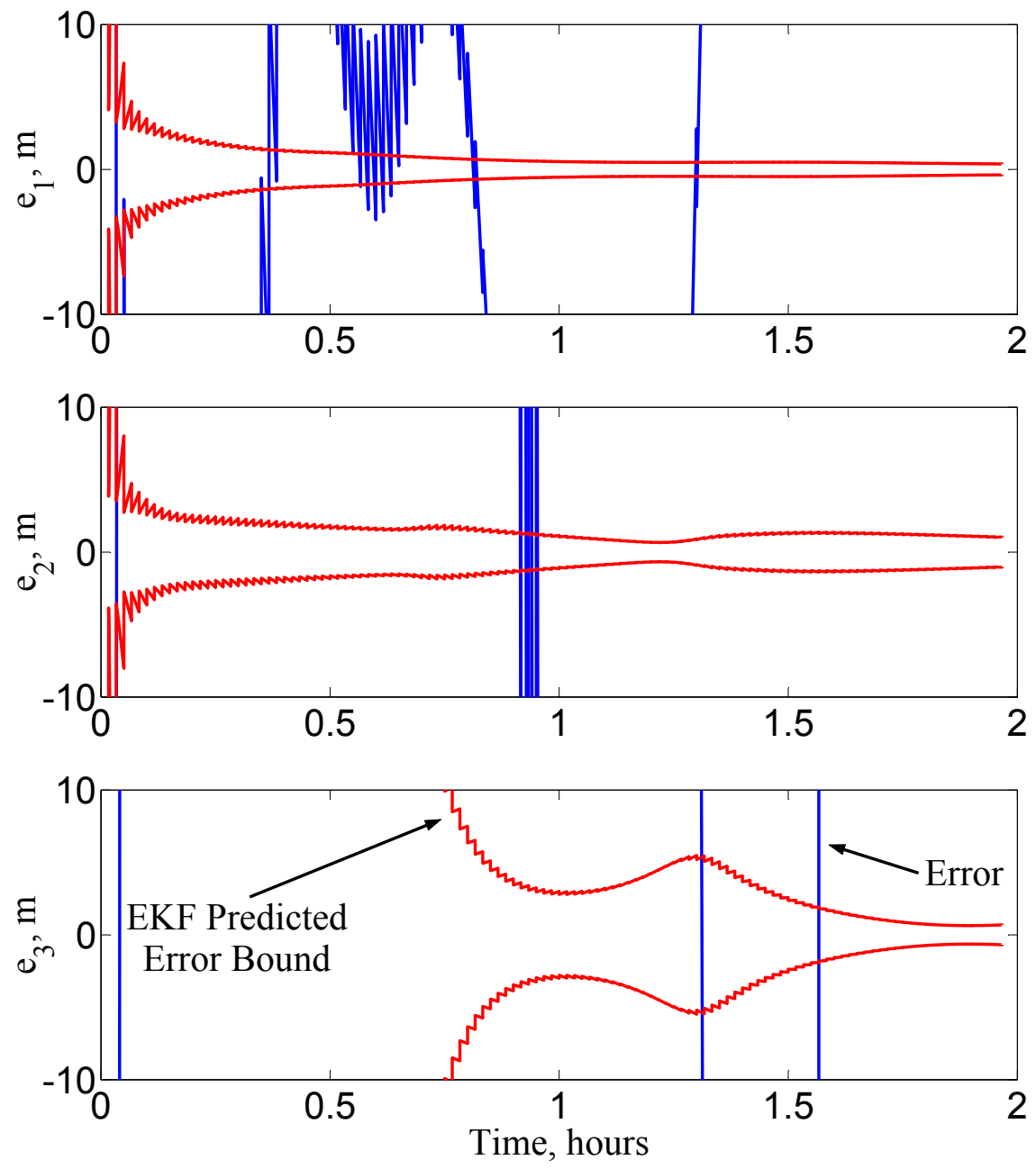

Figure 5.1: Time history of the position errors for Case 3.1, Scheme 0.

covariance is unacceptable.

These results beg the question, what is occurring in Case 3.1 that causes 

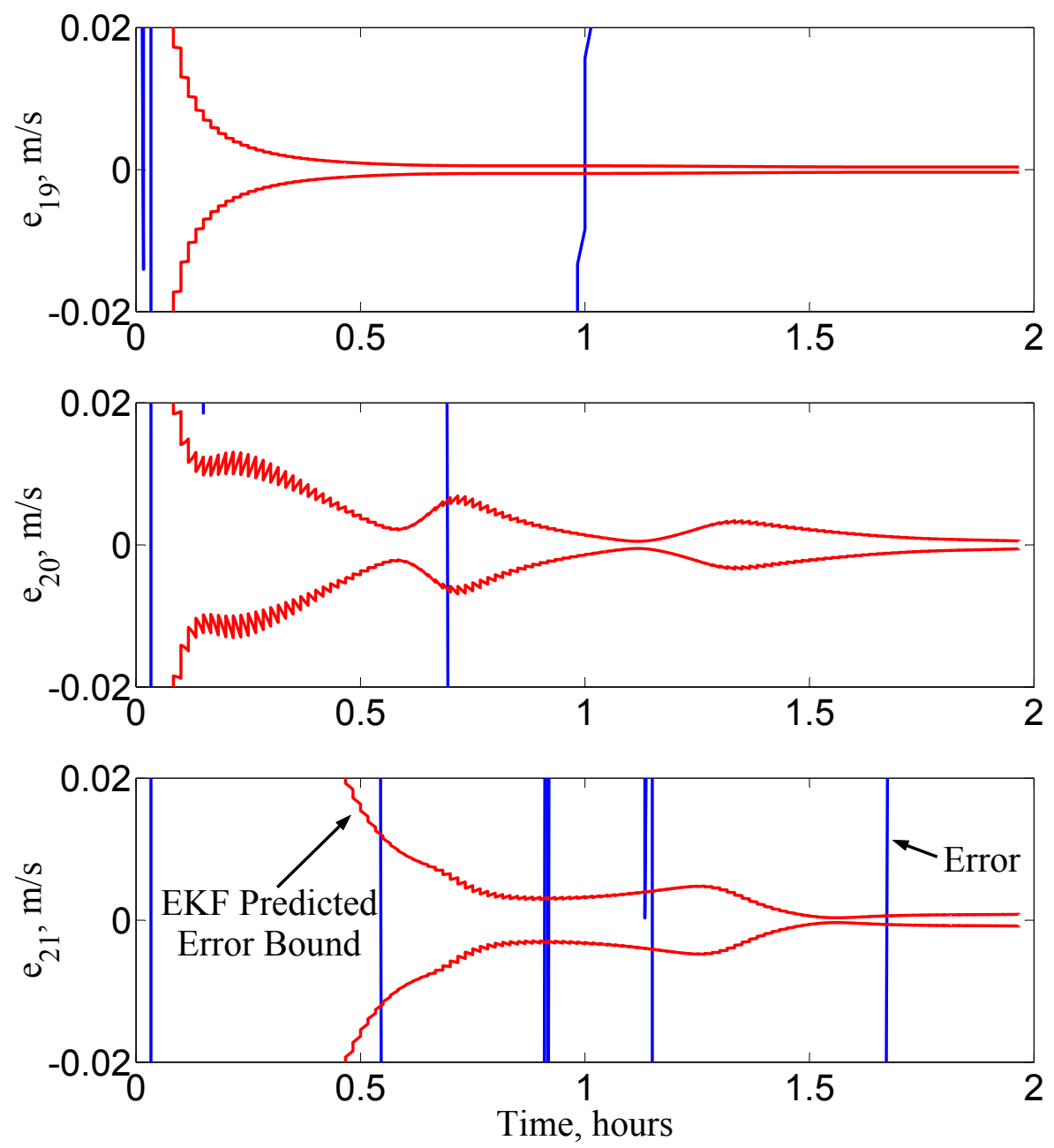

Figure 5.2: Time history of the velocity errors for Case 3.1, Scheme 0.

the filter to be overly sensitive to the fusion of relative measurements? Or more precisely, why are the nonlinearities of the relative measurements much more 
significant than the inertial measurements? One response to these questions is to examine the relationship between the state error covariance and the relative measurement residuals and residual covariances. Based on the state described in Eq. (2.17), the state error covariance is of the form,

$$
\mathbf{P}=\left[\begin{array}{l|l}
\mathbf{P}_{\mathbf{r r}} & \mathbf{P}_{\mathbf{r v}} \\
\hline \mathbf{P}_{\mathbf{v r}} & \mathbf{P}_{\mathbf{v v}}
\end{array}\right] \quad \text { where } \quad \mathbf{P}_{\mathbf{v r}}^{T}=\mathbf{P}_{\mathbf{r v}}=\left[\begin{array}{ccc}
\mathbf{P}_{\mathbf{r}_{1} \mathbf{v}_{1}} & \cdots & \mathbf{P}_{\mathbf{r}_{1} \mathbf{v}_{4}} \\
\vdots & \ddots & \vdots \\
\mathbf{P}_{\mathbf{r}_{4} \mathbf{v}_{1}} & \cdots & \mathbf{P}_{\mathbf{r}_{4} \mathbf{v}_{4}}
\end{array}\right]
$$

and

$$
\mathbf{P}_{\mathbf{r r}}=\left[\begin{array}{ccc}
\mathbf{P}_{\mathbf{r}_{1} \mathbf{r}_{1}} & \cdots & \mathbf{P}_{\mathbf{r}_{1} \mathbf{r}_{4}} \\
\vdots & \ddots & \vdots \\
\mathbf{P}_{\mathbf{r}_{4} \mathbf{r}_{1}} & \cdots & \mathbf{P}_{\mathbf{r}_{4} \mathbf{r}_{4}}
\end{array}\right] \quad \text { and } \quad \mathbf{P}_{\mathbf{v v}}=\left[\begin{array}{ccc}
\mathbf{P}_{\mathbf{v}_{1} \mathbf{v}_{1}} & \cdots & \mathbf{P}_{\mathbf{v}_{1} \mathbf{v}_{4}} \\
\vdots & \ddots & \vdots \\
\mathbf{P}_{\mathbf{v}_{4} \mathbf{v}_{1}} & \cdots & \mathbf{P}_{\mathbf{v}_{4} \mathbf{v}_{4}}
\end{array}\right]
$$

The relative position error covariance between the $i^{\text {th }}$ and $j^{\text {th }}$ spacecraft can be written as

$$
\mathbf{P}_{\mathbf{r}_{i} \mathbf{r}_{j}}^{r e l}=\mathbf{P}_{\mathbf{r}_{i} \mathbf{r}_{i}}+\mathbf{P}_{\mathbf{r}_{j} \mathbf{r}_{j}}-\mathbf{P}_{\mathbf{r}_{i} \mathbf{r}_{j}}-\mathbf{P}_{\mathbf{r}_{j} \mathbf{r}_{i}}
$$

It is also convenient to define a generic position covariance $\mathbf{P}_{\text {pos }}$ that is unique to each measurement type yielding

$$
\mathbf{P}_{p o s}:= \begin{cases}\mathbf{P}_{\mathbf{r}_{j} \mathbf{r}_{j}} & 1 \leq k \leq m_{i n} \\ \mathbf{P}_{\mathbf{r}_{i} \mathbf{r}_{j}}^{r e l} & k>m_{\text {in }}\end{cases}
$$

Recalling Eq. (3.33), it follows that Eq. (5.4) can be used to obtain a generic expression for the residual covariance of any range measurement (inertial or relative). In particular, the EKF residual covariance for measurement $h_{k}=\rho_{i j}$ (for any $k$ ) is

$$
W_{k k}^{e k f}=\frac{\boldsymbol{\rho}_{i j}^{T} \mathbf{P}_{p o s}^{-} \boldsymbol{\rho}_{i j}}{\rho_{i j}^{2}}+R_{k k}
$$


Since $\mathbf{P}_{\text {pos }}^{-}$is positive definite symmetric, by definition the quadratic ratio term in Eq. (5.5) must be positive. Furthermore, using the Rayleigh-Ritz Theorem [25] it can then be shown that

$$
0<\frac{\boldsymbol{\rho}_{i j}^{T} \mathbf{P}_{p o s}^{-} \boldsymbol{\rho}_{i j}}{\rho_{i j}{ }^{2}} \leq\left\|\mathbf{P}_{p o s}^{-}\right\|
$$

for all $\boldsymbol{\rho}_{i j}$.

Using these results, the relationship between the position covariance and the residuals and residual covariances have been examined for each of the four cases using the $\operatorname{EKF}\left(\mathbf{b}_{h}=\mathbf{0}\right.$ and $\left.\mathbf{B}_{h}=\mathbf{0}\right)$. As might be expected when relative range measurements are fused, the 2-norm of the relative position covariance $\left\|\mathbf{P}_{\mathbf{r}_{i} \mathbf{r}_{j}}^{r e l}\right\|$ reduces much more quickly than without the use of relative measurements. Consequently as Eq. (5.6) implies, for $k>m_{\text {in }}$ and a constant $R_{k k}$, the residual covariance $W_{k k}^{e k f}$ must also reduce rapidly. However, for Case 3.1, despite this rapid decrease in the relative range residual covariance, the magnitude of the EKF residual $\left|\epsilon_{k}^{e k f}\right|$ remains relatively large. This inconsistency between the residual and residual covariance leads to an inaccurate state error covariance, which in turn results in filter divergence. Thus in this case it is desirable to increase the residual covariance such that its convergence rate is consistent with that of the residual.

\subsection{First-Order Robustness Enhancement Algorithms}

As noted previously, the following four first-order robustness enhancement schemes are examined for Case 3.1: (A) Scalar Update Scheme, (B) 
Process Noise Scheme, (C) Measurement Noise Scheme, and (D) Selective Measurement Scheme.

\subsubsection{Scalar Update Scheme}

As noted in [27] and experienced in Section 4.2, when considering a highly observable system, the scalar update appears to be more stable than the matrix update when fusing relative measurements, so long as all the inertial measurements are processed first. The scalar (or "row-by-row") update is so named because it process the scalar components of the measurement vector one (row) at a time. This is accomplished by considering the simultaneous measurement components to occur consecutively over an infinitesimal span of time. Modifying the EKF matrix update equations in this way results in the algorithm presented in Table 5.3, where $\mathbf{H}_{k}$ refers to the $k^{t h}$ row of the $m \times n$ matrix $\mathbf{H}, \epsilon_{k}$ refers to the $k^{\text {th }}$ component of the $m \times 1$ residual vector $\boldsymbol{\epsilon}$, and $R_{k k}$ refers to the $k^{t h}$ diagonal element of the $m \times m$ matrix $\mathbf{R}$. Alternatively, Crassidis [14] offers a variation of this algorithm for the Kalman filter that updates the state error covariance in this sequential manner but only updates the state estimate after all the measurements have been processed.

This method inherently causes the order in which measurements are processed to become significant. In particular, since the state estimate is updated as each scalar measurement is processed, each subsequent linearization of the measurement model is about a more accurate state estimate, which in turn results in a reduced state error covariance. Consequently, if in the initial 
Table 5.3: Scalar Update Algorithm

$$
\begin{aligned}
& \text { Given: } \hat{\mathbf{x}}^{-}, \mathbf{P}^{-}, \mathbf{z} \text {, and } \mathbf{R} \text { at time } t_{d} \\
& \text { FOR } k=1, \ldots, m \\
& \quad \epsilon_{k}=z_{k}-h_{k}\left(\hat{\mathbf{x}}^{-}\right) \text {and } \mathbf{H}_{k}=\mathbf{H}_{k}\left(\hat{\mathbf{x}}^{-}\right) \\
& \mathbf{K}=\mathbf{P}^{-} \mathbf{H}_{k}^{T} /\left(\mathbf{H}_{k} \mathbf{P}^{-} \mathbf{H}_{k}^{T}+R_{k k}\right) \\
& \hat{\mathbf{x}}^{+}=\hat{\mathbf{x}}^{-}+\mathbf{K} \epsilon_{k} \\
& \quad \mathbf{P}^{+}=\left(\mathbf{I}-\mathbf{K} \mathbf{H}_{k}\right) \mathbf{P}^{-}\left(\mathbf{I}-\mathbf{K H}_{k}\right)^{T}+R_{k k} \mathbf{K} \mathbf{K}^{T} \\
& \hat{\mathbf{x}}^{-}=\hat{\mathbf{x}}^{+} \text {and } \mathbf{P}^{-}=\mathbf{P}^{+} \\
& \text {END FOR }(k) \\
& \text { Set: } \hat{\mathbf{x}}^{+}=\hat{\mathbf{x}}^{-} \text {and } \mathbf{P}^{+}=\mathbf{P}^{-}
\end{aligned}
$$

update, processing the inertial measurements first can successfully reduce the state error covariance from a severe level to a moderate level, then the potential for fusion complications is eliminated before the relative measurements are even processed. However, while this would result in filter convergence, this scheme is at best circumstantial and at worst only feasible in the rare instance that the relative measurements are never processed in the presence of a severe level of state error covariance. In other words, in a less observable system the initial processing of the inertial measurements may not result in a significant reduction in the state error covariance. While the scalar update scheme works for the Case 3.1 mission, which uses three inertial measurements, in the single inertial measurement mission presented in [28], this scheme is not adequate to prevent filter divergence. The scalar update scheme has similarly been exploited in some radar tracking algorithms where the azimuth measurements, which have insignificant nonlinearities in practical tracking environments, are processed prior to the range measurements [44]. However, once again, this 
scheme yields degraded performance when the nonlinearities become more significant.

\subsubsection{Process and Measurement Noise Schemes}

The next two schemes presented are extensions of the standard filter tuning procedure in which the filter noise is "tuned" to the appropriate value. One tuning parameter of this procedure is the process noise. As implied in Section 2.3.2, since this simulation uses the same dynamical model for the environment and the filter, in general process noise is not required. Nevertheless it is not uncommon in practice to add artificial "pseudo-noise" to prevent filter divergence due to numerical inadequacies. Employing this strategy, Case 3.1 was reexamined with the inclusion of process noise (i.e. $\mathbf{Q}_{\text {spec }} \neq \mathbf{0}$ ). After evaluating several different levels of process noise in the simulation it was determined that in order to prevent filter divergence the process noise would need to be so extreme that the filter performance was worse than simply not processing the relative measurements (i.e. Case 3.0 without process noise). Although this scheme prevents filter divergence, since the overall goal is to fuse the relative measurements to obtain a more accurate state estimate, using this scheme is ultimately counterproductive.

Another parameter of the filter tuning procedure is the adjustment of the measurement noise. In practice the true noise associated with a particular measurement is not necessarily accurately known. However, in general the larger the measurement noise covariance, the less confidence the filter has in 
the measurement. In this way measurements can slowly be brought "online" by intentionally giving the filter an initially exaggerated measurement noise covariance and then gradually reducing it until a more realistic value is reached. For example, this could be done for the relative measurements in Case 3.1 by initially setting $R_{k k}\left(t_{1}\right)={\tilde{\sigma_{\rho}}}^{2}$ where $k>m_{\text {in }}$ and $\tilde{\sigma}_{\rho} \gg \sigma_{\rho}$, and then reducing $\tilde{\sigma}_{\rho}$ until it is comparable to $\sigma_{\rho}$. This reduction in measurement noise covariance can be achieved by some chosen functional reduction method (e.g. $\left.R_{k k}=R_{k k}(t)\right)$ or by using an adaptive measurement noise algorithm. Also note that, as seen in Eq. (5.5), increasing the measurement noise covariance $R_{k k}$ has the desired effect of increasing the residual covariance $W_{k k}$. Several different systematically reduced and adaptive measurement noise schemes have been evaluated for this purpose. In particular (for $\tilde{\sigma}_{\rho}^{2}\left(t_{0}\right)=50 \mathrm{~km}^{2}$ ), the functional method used an exponential function of time to reduce the relative measurement noise covariance, while the adaptive algorithm was based on a combination of the algorithm presented in Maybeck [40] and the runningaverage filter presented in Busse, et al. [9].

The running-average (or fading memory) filter described in Table 5.4 is used to incorporate the measurement noise covariance update $R_{k k}^{*}$ into the previous measurement noise covariance. In Table 5.4, $m_{\text {in }}$ is the number of inertial measurements $\left(m_{i n}=3 p\right), w$ is the length of the memory window of the past measurements to be averaged $(w=20)$, and the last IF statement is to prevent the measurement noise covariance from dropping below the variance of the actual measurement noise. The full matrix equation for the update $\mathbf{R}^{*}$ 
Table 5.4: Adaptive Measurement Noise Algorithm

Given: $\mathbf{R}^{*}$ at times $t_{d}$ and $t_{d-w}$ using Eq. (5.7)

FOR $k=m_{\text {in }}+1, \ldots, m$

IF $d \leq w$

$$
R_{k k}\left(t_{d}\right)=R_{k k}\left(t_{d-1}\right)+\frac{R_{k k}^{*}\left(t_{d}\right)-R_{k k}\left(t_{d-1}\right)}{d}
$$

ELSE

$$
R_{k k}\left(t_{d}\right)=R_{k k}\left(t_{d-1}\right)+\frac{R_{k k}^{*}\left(t_{d}\right)-R_{k k}^{*}\left(t_{d-w}\right)}{w}
$$

END IF

IF $R_{k k}\left(t_{d}\right)<\sigma_{\rho}^{2}$

$R_{k k}\left(t_{d}\right)=\sigma_{\rho}^{2}$

END IF

$\operatorname{END} \operatorname{FOR}(k)$

at discrete measurement time $t_{d}$ can be written as,

$$
\mathbf{R}^{*}=\boldsymbol{\epsilon} \boldsymbol{\epsilon}^{T}+\mathbf{H P}^{+} \mathbf{H}^{T}
$$

where $\boldsymbol{\epsilon}, \mathbf{H}$, and $\mathbf{P}^{+}$have been obtained using the a posteriori values at the time of the previous measurement (i.e. $\boldsymbol{\epsilon}=\mathbf{z}\left(t_{d-1}\right)-\mathbf{h}\left(\hat{\mathbf{x}}\left(t_{d-1}^{+}\right)\right), \mathbf{H}=$ $\mathbf{H}\left(\hat{\mathbf{x}}\left(t_{d-1}^{+}\right)\right)$, and $\left.\mathbf{P}^{+}=\mathbf{P}\left(t_{d-1}^{+}\right)\right)$. Both of these methods demonstrated not only the capacity to prevent filter divergence in Case 3.1, but also the ability to decrease the navigation error by correctly incorporating the relative measurements. A summary of the performance of the adaptive method, which performed better and required far less tuning, is available in Table 5.5 (I-C). Both of these methods achieve this performance by tuning the relative measurement noise such that the filter essentially ignores the relative measurements until the state error covariance is reduced to a moderate level. Consequently, if similarly 
tuned these schemes offer similar performance to the selective measurement scheme.

\subsubsection{Selective Measurement Scheme}

The selective measurement scheme, which has also been used in practice, takes a more direct approach by not introducing the relative measurements until after the state error covariance has converged to an acceptable level (e.g. a moderate level). For example, this can be accomplished by not processing relative range measurements unless the square root of the maximum diagonal elements of $\mathbf{P}_{\mathbf{r r}}$ and $\mathbf{P}_{\mathbf{v v}}$ are less than the moderate values of $\sigma_{\mathbf{r}}$ and $\sigma_{\mathbf{v}}$, respectively, presented in Table 5.1. Such a scheme is similar to implementing the scalar update scheme with a residual check that instead ignores measurements only when they violate the residual covariance inconsistency. For example, if $\epsilon_{k}^{2} / W_{k k}>$ tol, where tol is some predetermined tolerance, the $k^{\text {th }}$ measurement $z_{k}$ would be ignored by setting $\mathbf{K}=\mathbf{0}$ at the appropriate step in Table 5.3. However, unlike the selective measurement scheme that has demonstrated effective performance for Case 3.1, the residual check in its current form has not proven to be a viable solution for this particular application. The performance of the selective measurement scheme is summarized in Table $5.5(\mathrm{I}-\mathrm{D})$.

Although the selective measurement scheme and the adaptive measurement noise scheme have been proven successful, they share two unfortunate traits. First, they require the tuning of an initial parameter or tolerance. In 
general, filter tuning is problem specific and at times is extremely tedious. This can be especially true in this situation where there is a constant tradeoff between robustness and bringing the relative measurements online in a timely manner. Also, even if the tuning turns out to be fairly straightforward, these schemes still do not guarantee to be suitably robust outside of that particular tuning regime. Furthermore, in order to optimize efficiency, the selective measurement scheme would require a comprehensive analysis to determine the ideal state error covariance tolerance and a robust automated implementation

method. Finally, the second trait shared by these schemes is that although they avoid the effects of the fusion complications, they do not yield any additional insight into the cause of these complications. For this reason the second-order terms normally truncated during the first-order Taylor series expansion have been thoroughly examined.

\subsection{Second-Order Robustness Enhancement Algorithms}

The focus of investigating second-order methods is to determine what significance the second-order terms resulting from quadratic nonlinearities that are normally truncated have in regards to the experienced fusion complications. Maybeck states that "...empirical results have corroborated that nonlinear effects are most important when noise inputs are small while estimation error variance is relatively large." [40] This is exactly the scenario presented in Case 3.1 when no robustness enhancement schemes are implemented. The first-order noise schemes exploit this fact by using noise to account for model 
inadequacies due to neglecting higher order terms. To verify this, Case 3.1 was reexamined with the implementation of the GSF in place of using the EKF with artificial or exaggerated noise. The resulting performance is summarized in Table 5.5 (II). Notice that while the GSF outperforms the first-order methods the results are quite comparable. This indicates that the measurements noise scheme is doing an adequate job at accounting for the second-order terms that the EKF neglects. The selective measurement scheme on the other hand accomplishes this by not introducing the relative measurements until the second-order terms have become negligible. Finally, while in this case the slight increase in accuracy achieved by the GSF does come at a cost of roughly a $50 \%$ increase in computation time, it does have the added benefit of general robustness without any required filter tuning. Furthermore, as will be shown later, if the second-order state estimate propagation term is not required (i.e. $\mathbf{b}_{f}=\mathbf{0}$ ), there is only about a $20 \%$ increase in computation time.

The challenge is to determine why the second-order terms are so much more significant when the relative measurements are fused. To accomplish this Eqs. (2.22) and (5.1) are used to reduce Eq. (3.23) to

$$
b_{f_{i}}=\frac{1}{2} \operatorname{trace}\left(\boldsymbol{\Delta}_{f_{i}} \mathbf{P}_{\mathbf{r r}}\right)= \begin{cases}0 & 1 \leq i \leq n / 2 \\ \frac{1}{2} \operatorname{trace}\left(\mathbf{G}_{j k}^{\prime} \mathbf{P}_{\mathbf{r}_{j} \mathbf{r}_{j}}\right) & n / 2<i \leq n\end{cases}
$$

where the subscript $j$ refers to the spacecraft number $(j=1, \ldots, 4)$, the subscript $k$ refers to the $x, y$, or, $z$ component of $\mathbf{v}_{j}$ or $\mathbf{g}_{j}(k=1,2,3$, respectively) corresponding to $f_{i}$ and $\mathbf{G}_{j k}^{\prime}$ is defined in Eq. (2.23). Likewise, using Eqs. 
(2.28) and (5.1), Eqs. (3.30) and (3.34) can be reduced to,

$$
b_{h_{k}}=\frac{1}{2} \operatorname{trace}\left(\boldsymbol{\Delta}_{h_{k}} \mathbf{P}_{\mathbf{r r}}^{-}\right)=\frac{1}{2} \operatorname{trace}\left(\boldsymbol{\Theta}_{i j}^{\prime} \mathbf{P}_{\text {pos }}^{-}\right)
$$

and

$$
B_{h_{k k}}=\frac{1}{2} \operatorname{trace}\left(\boldsymbol{\Delta}_{h_{k}} \mathbf{P}_{\mathbf{r r}}^{-} \boldsymbol{\Delta}_{h_{k}} \mathbf{P}_{\mathbf{r r}}^{-}\right)=\frac{1}{2} \operatorname{trace}\left(\boldsymbol{\Theta}_{i j}^{\prime} \mathbf{P}_{p o s}^{-} \boldsymbol{\Theta}_{i j}^{\prime} \mathbf{P}_{\text {pos }}^{-}\right)
$$

where the subscript $j$ refers to the primary spacecraft number, while the subscript $i$ refers to the station number (for inertial measurements) or the secondary spacecraft number (for relative measurements) that is tracking the primary; and $\boldsymbol{\Theta}_{i j}^{\prime}$ and $\mathbf{P}_{\text {pos }}^{-}$are defined in Eqs. (2.29) and (5.4), respectively.

The following theorem adapted from Horn [25] will be useful:

Theorem 5.3.1. For any two symmetric positive semidefinite matrices $\mathbf{A}$ and $\mathbf{B}$, the matrix $\mathbf{A B}$ has real nonnegative eigenvalues. Consequently,

$$
\operatorname{trace}(\mathbf{A B})=\sum_{i} \lambda_{i}(\mathbf{A B}) \geq 0 \quad \text { and } \quad 0 \leq \operatorname{trace}(\mathbf{A B A B}) \leq(\operatorname{trace}(\mathbf{A B}))^{2}
$$

Anticipating that Theorem 5.3.1 will require a symmetric positive semidefinite matrix, it is also useful to define the matrix $(\|\mathbf{A}\| \mathbf{I}-\mathbf{A})$, which for any symmetric $\mathbf{A}$ is symmetric positive semidefinite by Theorem 5.3.2; where as described in Section 2.1, in general $\|\cdot\|$ indicates the vector or matrix 2-norm and $\lambda_{i}(\cdot)$ indicates the $i^{\text {th }}$ component of the corresponding vector of eigenvalues, $\boldsymbol{\lambda}(\cdot)$.

Theorem 5.3.2. For any symmetric matrix $\mathbf{A}$ the matrix $(\|\mathbf{A}\| \mathbf{I}-\mathbf{A})$ is symmetric positive semidefinite. 
Proof.

$$
\begin{aligned}
\lambda_{i}(\|\mathbf{A}\| \mathbf{I}-\mathbf{A}) & =\|\mathbf{A}\|-\lambda_{i}(\mathbf{A}) \\
& =\max |\boldsymbol{\lambda}(\mathbf{A})|-\lambda_{i}(\mathbf{A}) \\
& \geq 0
\end{aligned}
$$

Finally, combining Theorems 5.3.1 and 5.3.2 the following theorem, adapted from Ran and Reurings [45], is obtained:

Theorem 5.3.3. For any symmetric matrix $\mathbf{A}$ and symmetric positive semidefinite matrix $\mathbf{B}$,

$$
\operatorname{trace}(\mathbf{A B}) \leq\|\mathbf{A}\| \operatorname{trace}(\mathbf{B})
$$

Proof.

$$
\begin{aligned}
\operatorname{trace}((\|\mathbf{A}\| \mathbf{I}-\mathbf{A}) \mathbf{B}) & \geq 0 \quad(\text { Thms. 5.3.1 and 5.3.2) } \\
\operatorname{trace}(\|\mathbf{A}\| \mathbf{B}-\mathbf{A B}) & \geq 0 \\
\|\mathbf{A}\| \operatorname{trace}(\mathbf{B})-\operatorname{trace}(\mathbf{A B}) & \geq 0 \\
\|\mathbf{A}\| \operatorname{trace}(\mathbf{B}) & \geq \operatorname{trace}(\mathbf{A B})
\end{aligned}
$$

Recognizing that $\mathbf{G}_{j k}^{\prime}$ is symmetric and $\mathbf{P}_{\mathbf{r}_{j} \mathbf{r}_{j}}$ is symmetric positive definite, applying Theorem 5.3.3 to Eq. (5.8) results in the following bound on $b_{f_{i}}$ for all $i>n / 2$ :

$$
\left|b_{f_{i}}\right| \leq \frac{1}{2}\left\|\mathbf{G}_{j k}^{\prime}\right\| \operatorname{trace}\left(\mathbf{P}_{\mathbf{r}_{j} \mathbf{r}_{j}}\right)
$$

Likewise, since $\Theta_{i j}^{\prime}$ is symmetric and positive semidefinite by Eq. (5.15) and $\mathbf{P}_{\text {pos }}^{-}$is symmetric positive definite, Theorems 5.3.1 and 5.3.3 can be applied to Eq. (5.9) to obtain bounds for $b_{h_{k}}$ yielding

$$
0 \leq b_{h_{k}} \leq \frac{1}{2}\left\|\Theta_{i j}^{\prime}\right\| \operatorname{trace}\left(\mathbf{P}_{\text {pos }}^{-}\right)
$$


and applied to Eq. (5.10) to obtain bounds for $B_{h_{k k}}$, as

$$
0 \leq B_{h_{k k}} \leq \frac{1}{2}\left(\left\|\boldsymbol{\Theta}_{i j}^{\prime}\right\| \operatorname{trace}\left(\mathbf{P}_{p o s}^{-}\right)\right)^{2}
$$

Notice that since $B_{h_{k k}} \geq 0$, it has the desired effect of increasing the relative range residual covariance $W_{k k}$ (see Eq. (3.33)). Next, since $\mathbf{G}_{j k}^{\prime}$ and $\boldsymbol{\Theta}_{i j}^{\prime}$ are symmetric, their 2-norm is the maximum of the absolute value of their eigenvalues. Thus, computing the eigenvalues of $\mathbf{G}_{j k}^{\prime}$ and $\boldsymbol{\Theta}_{i j}^{\prime}$ results in

$$
\boldsymbol{\lambda}\left(\mathbf{G}_{j k}^{\prime}\right)=\frac{3 \mu}{2\left\|\mathbf{r}_{j}\right\|^{5}}\left[\begin{array}{c}
2 r_{j_{k}} \\
-r_{j_{k}}+\sqrt{5 r_{j_{k}}^{2}+4\left\|\mathbf{r}_{j}\right\|^{2}} \\
-r_{j_{k}}-\sqrt{5 r_{j_{k}}^{2}+4\left\|\mathbf{r}_{j}\right\|^{2}}
\end{array}\right] \quad, \quad\left\|\mathbf{G}_{j k}^{\prime}\right\| \leq \frac{6 \mu}{\left\|\mathbf{r}_{j}\right\|^{4}}
$$

and

$$
\boldsymbol{\lambda}\left(\boldsymbol{\Theta}_{i j}^{\prime}\right)=\frac{1}{\rho_{i j}}\left[\begin{array}{l}
1 \\
1 \\
0
\end{array}\right] \quad, \quad\left\|\Theta_{i j}^{\prime}\right\|=\frac{1}{\rho_{i j}}
$$

Notice that while an equality for $\left\|\mathbf{G}_{j k}^{\prime}\right\|$ cannot be determined in general, the term can still be conservatively bounded. These results indicate that the bounds on the second-order terms are functions solely of the trace of the appropriate state error covariance matrix and the orbit radius magnitude $\left\|\mathbf{r}_{j}\right\|$ or the range measurement magnitude $\rho_{i j}$.

To verify the bound on $b_{f_{i}}$, Eq. (5.8) was evaluated for various random values of $\mathbf{P}_{\mathbf{r}_{j} \mathbf{r}_{j}}$ and $\mathbf{r}_{j}$, for a set $\left\|\mathbf{r}_{j}\right\|=R_{\zeta}$. This particular constraint value was chosen because it allows for the maximum value obtainable by $b_{f_{i}}$. According to Eq. (5.14), the upper bound on $\left\|\mathbf{G}_{j k}^{\prime}\right\|$ is maximized by minimizing

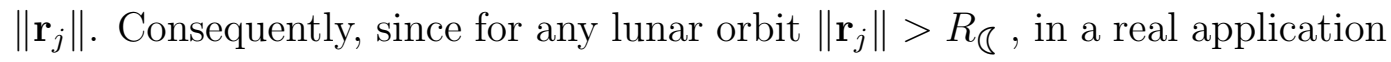
$\left\|\mathbf{G}_{j k}^{\prime}\right\|$, and thus $b_{f_{i}}$, will never achieve values higher then those computed with 


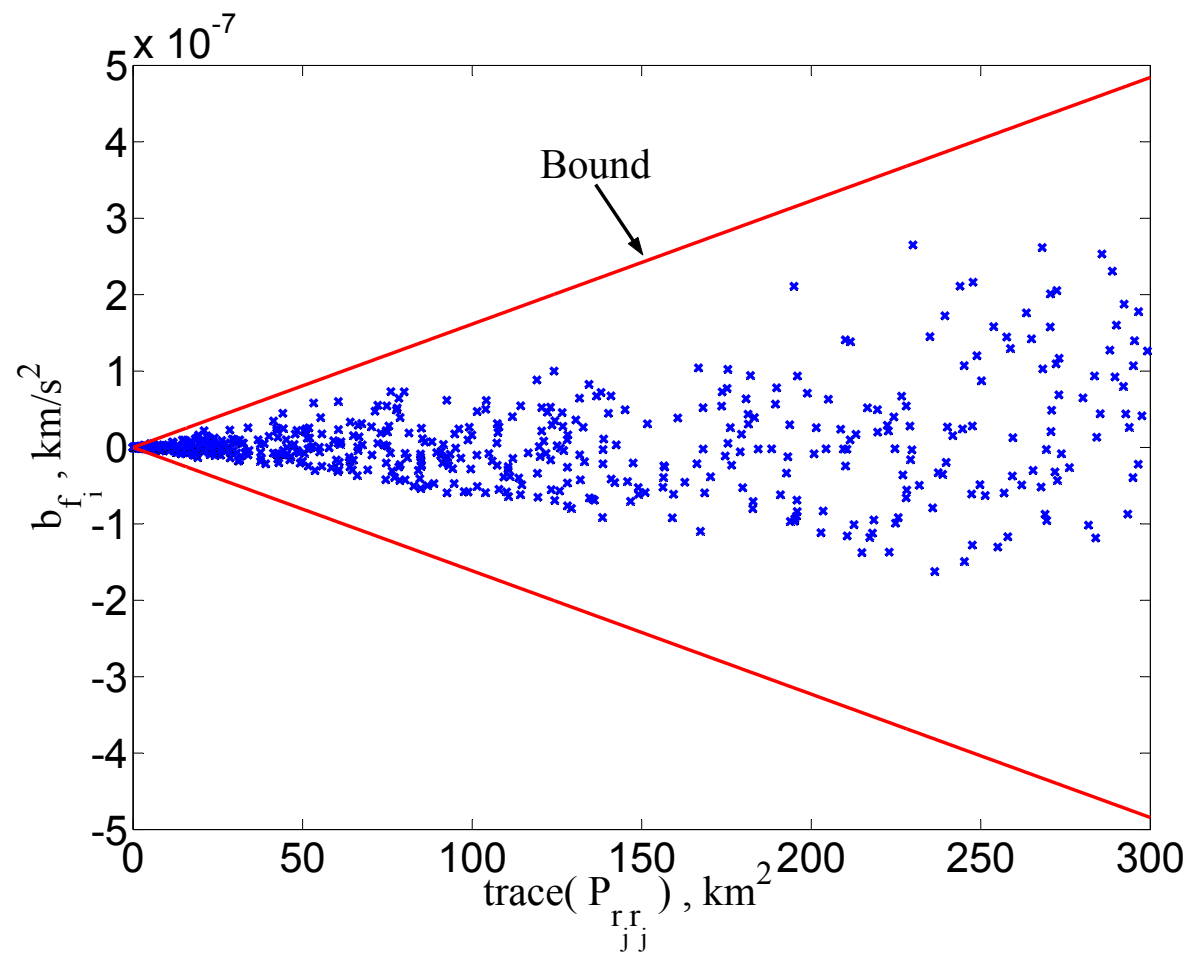

Figure 5.3: Evaluation of second-order term $b_{f_{i}}$ for $\left\|\mathbf{r}_{j}\right\|=R_{\zeta}$ and random $\mathbf{P}_{\mathbf{r}_{j} \mathbf{r}_{j}}$ with the corresponding bounds of Eq. (5.11).

$\left\|\mathbf{r}_{j}\right\|=R_{\zeta}$. Thus, as illustrated by the solid lines in Figure 5.3, $\left\|b_{f_{i}}\right\|$ is in fact conservatively bounded by Eq. (5.11).

Similarly, to verify the bounds on $b_{h_{k}}$ and $B_{h_{k k}}$, Eqs. (5.9) and (5.10) were evaluated for various random values of $\mathbf{P}_{p o s}^{-}$and $\boldsymbol{\rho}_{i j}$, for a set $\left\|\boldsymbol{\rho}_{i j}\right\|=\rho_{i j}$. This time the random values of $\boldsymbol{\rho}_{i j}$ were constrained using multiple values of $\rho_{i j}$ to illustrate the sensitivity of $b_{h_{k}}$ and $B_{h_{k k}}$ to the range measurement magnitude. In particular it was desirable to compare inertial range measure- 

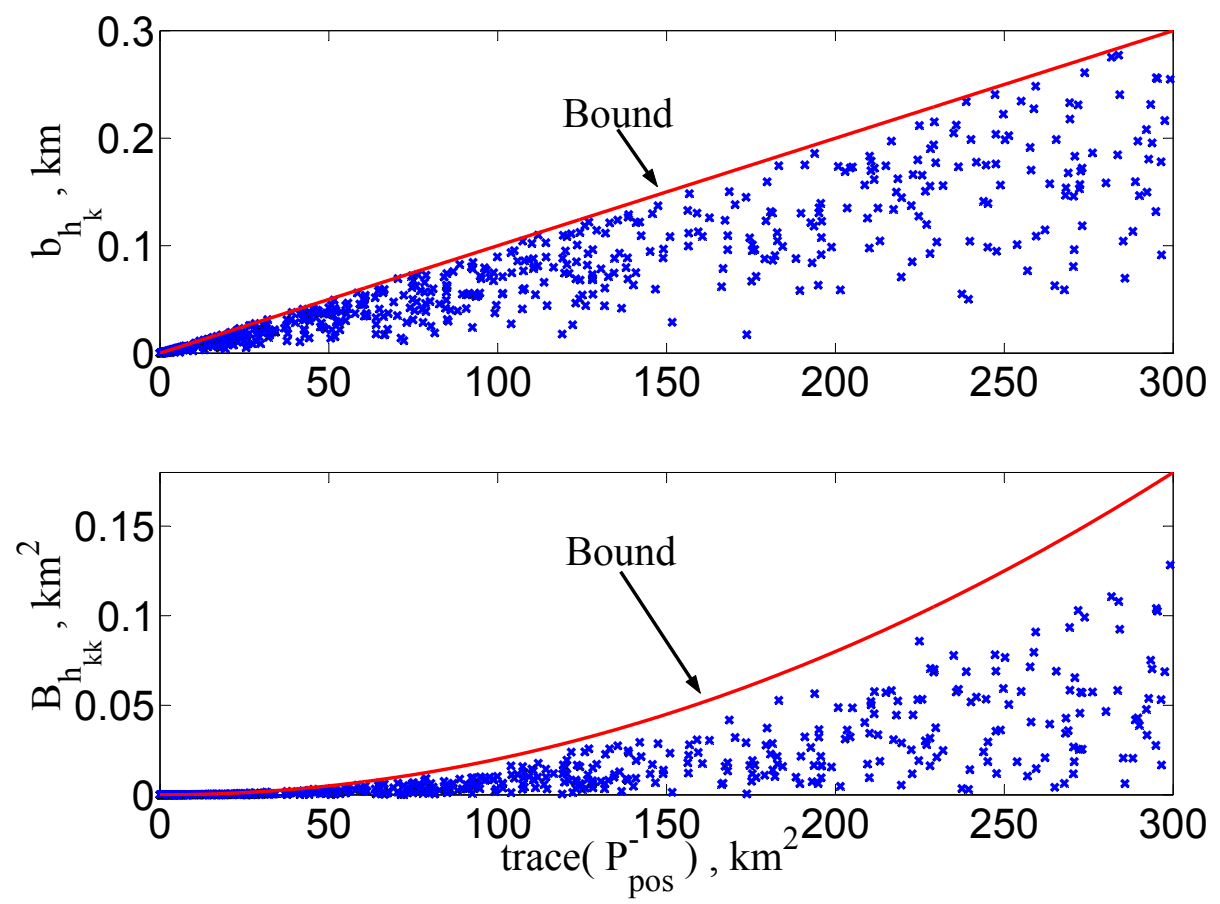

Figure 5.4: Evaluation of second-order terms $b_{h_{k}}$ and $B_{h_{k k}}$ for $\rho_{i j}=500 \mathrm{~km}$ and random $\mathbf{P}_{\text {pos }}^{-}$with the corresponding upper bounds of Eqs. (5.12) and (5.13).

ment magnitudes with relative range measurement magnitudes. In general, as seen in Eq. (5.15), $\left\|\Theta_{i j}^{\prime}\right\|$ is maximized by minimizing $\rho_{i j}$. Thus, in order to demonstrate the maximum values obtainable for $b_{h_{k}}$ and $B_{h_{k k}}$ given an inertial range measurement, it is necessary to consider a constraint value that is on order with the smallest possible inertial range measurement. Although, as mentioned earlier, the smallest inertial range measurements considered in this scenario are on the order of $0.645 \times 10^{5} \mathrm{~km}$ (for $L_{2}$ ), suppose instead a lunar 

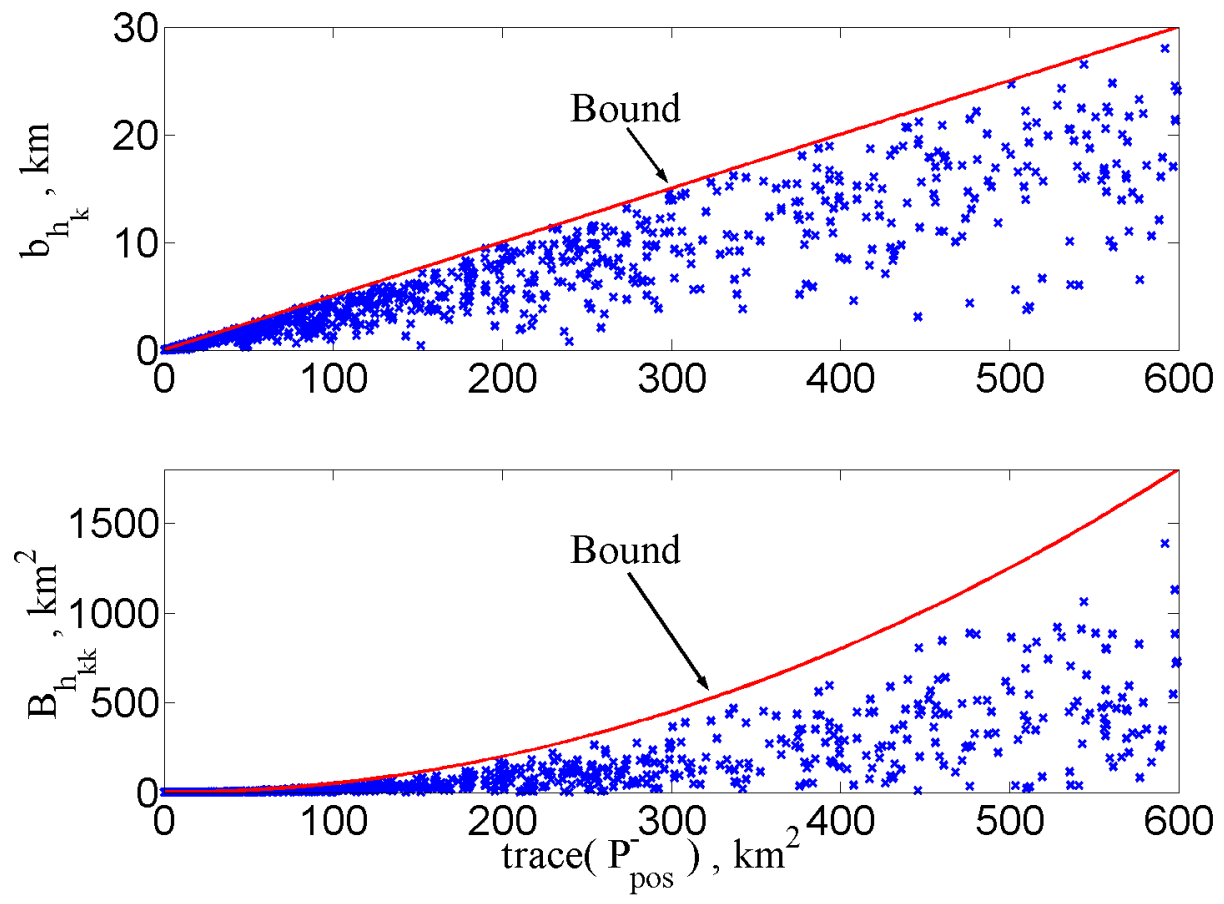

Figure 5.5: Evaluation of second-order terms $b_{h_{k}}$ and $B_{h_{k k}}$ for $\rho_{i j}=10 \mathrm{~km}$ and random $\mathbf{P}_{\text {pos }}^{-}$with the corresponding upper bounds of Eqs. (5.12) and (5.13).

surface beacon was available such that the smallest inertial range measurement was on the order of hundreds of kilometers. Due to this consideration a constraint value of $\rho_{i j}=500 \mathrm{~km}$ was chosen for the inertial range measurement magnitude.

Relative range measurements on the other hand, are not necessarily limited in magnitude. Depending on the mission, formation baselines between spacecraft can range from the order of 100's of meters to 100's of kilometers. 
Consequently, a constraint value equivalent to the most common relative range magnitude (between coplanar spacecraft) was chosen to be $\rho_{i j}=10 \mathrm{~km}$. Using these values, the solid lines in Figures 5.4 and 5.5 illustrate that $b_{h_{k}}$ and $B_{h_{k k}}$ are in fact bounded by Eqs. (5.12) and (5.13). Notice that even for the smallest possible inertial range measurement, the second-order terms for the relative range measurement are two orders of magnitude larger. Finally, as implied later, the trace values in Figure 5.5 are intentionally twice that of Figure 5.4 due to the corresponding definition of $\mathbf{P}_{\text {pos }}^{-}$in Eq. (5.4).

While Figures 5.3 to 5.5 demonstrate the range of possible values for the second-order terms, these values must be put into context to determine their significance. Thus, recalling Eq. (3.22) it is necessary to examine the order of $b_{f_{i}}$ relative to $f_{i}$. First, as is true in this case, suppose that initially $\mathbf{P}_{\mathbf{r}_{i} \mathbf{r}_{i}} \approx \mathbf{P}_{\mathbf{r}_{j} \mathbf{r}_{j}} \approx \sigma_{\mathbf{r}}^{2} \mathbf{I}$ and $\mathbf{P}_{\mathbf{r}_{i} \mathbf{r}_{j}} \approx \mathbf{P}_{\mathbf{r}_{j} \mathbf{r}_{i}} \approx \mathbf{0}$. Using these assumptions the "worst case" order of $\left|f_{i}\right|$ and $\left|b_{f_{i}}\right|$ can be approximated as,

$$
\begin{aligned}
& \left|f_{i}\right|=\left|g_{j_{k}}\right|=\frac{\mu}{\left\|\mathbf{r}_{j}\right\|^{3}}\left|r_{j_{k}}\right| \sim \frac{\mu}{\left\|\mathbf{r}_{j}\right\|^{2}} \\
& \left|b_{f_{i}}\right| \leq \frac{3 \mu}{\left\|\mathbf{r}_{j}\right\|^{4}} \operatorname{trace}\left(\mathbf{P}_{\mathbf{r}_{j} \mathbf{r}_{j}}\right) \sim \frac{9 \mu \sigma_{\mathbf{r}}^{2}}{\left\|\mathbf{r}_{j}\right\|^{4}}
\end{aligned}
$$

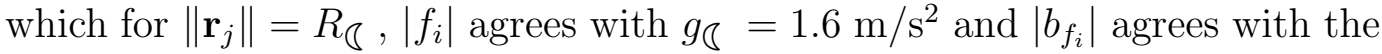
bounds in Figure 5.3. Furthermore, since $\left\|\mathbf{r}_{j}\right\|>R_{\overparen{\zeta}}$, even for a severe level of covariance $\left(\sigma_{\mathbf{r}} \sim 10 \mathrm{~km}\right)\left|b_{f_{i}}\right|$ will typically be at least four orders of magnitude smaller than $f_{i}$ and consequently negligible. This fact has been further demonstrated by successfully implementing the GSF in Case 3.1 without the second-order state estimate propagation term (i.e. $\left.\mathbf{b}_{f}=\mathbf{0}\right)$. This implies that 
the same GSF could easily be applied to higher fidelity gravity models. As shown in [28], these results similarly apply to Earth orbiting missions.

Likewise, recalling Eqs. (3.28) and (3.33) it is necessary to examine the order of $b_{h_{k}}$ relative to $\left|\epsilon_{k}^{e k f}\right|$ and $B_{h_{k k}}$ relative to $W_{k k}^{e k f}$ (see Eq. (5.5)). Based on the previous covariance assumptions, $\mathbf{P}_{\text {pos }}^{-}=\mathbf{P}_{\mathbf{r}_{j} \mathbf{r}_{j}} \approx \sigma_{\mathbf{r}}^{2} \mathbf{I}$ for inertial measurements $\left(1 \leq k \leq m_{\text {in }}\right)$ and $\mathbf{P}_{\text {pos }}^{-}=\mathbf{P}_{\mathbf{r}_{i} \mathbf{r}_{j}}^{r e l} \approx 2 \sigma_{\mathbf{r}}^{2} \mathbf{I}$ for relative measurements $\left(k>m_{i n}\right)$. Using these assumptions the "worst case" order of $\left|\epsilon_{k}^{e k f}\right|$ and $b_{h_{k}}$ can be approximated as,

$$
\begin{aligned}
\left|\epsilon_{k}^{e k f}\right| \sim \sqrt{W_{k k}^{e k f}} & \sim \begin{cases}\sigma_{\mathbf{r}} & 1 \leq k \leq m_{i n} \\
\sqrt{2} \sigma_{\mathbf{r}} & k>m_{\text {in }}\end{cases} \\
b_{h_{k}} \leq \frac{1}{2 \rho_{i j}} \operatorname{trace}\left(\mathbf{P}_{\text {pos }}^{-}\right) & \sim \begin{cases}3 \sigma_{\mathbf{r}}^{2} /\left(2 \rho_{i j}\right) & 1 \leq k \leq m_{i n} \\
3 \sigma_{\mathbf{r}}^{2} / \rho_{i j} & k>m_{i n}\end{cases}
\end{aligned}
$$

while the "worst case" order of $W_{k k}^{e k f}$ (for $R_{k k}=\sigma_{\rho}^{2} \ll \sigma_{\mathbf{r}}^{2}$ ) and $B_{h_{k k}}$ are approximated as,

$$
\begin{aligned}
W_{k k}^{e k f} & \sim \begin{cases}\sigma_{\mathbf{r}}^{2} & 1 \leq k \leq m_{i n} \\
2 \sigma_{\mathbf{r}}^{2} & k>m_{i n} \\
9 \sigma_{\mathbf{r}}^{4} /\left(2 \rho_{i j}^{2}\right) & 1 \leq k \leq m_{i n} \\
18 \sigma_{\mathbf{r}}^{4} / \rho_{i j}^{2} & k>m_{i n}\end{cases}
\end{aligned}
$$

all of which agree with the appropriate bounds in Figure 5.4 and 5.5.

As alluded to earlier, the significance of the second-order terms is now directly related to the magnitude of the range measurement, regardless of if it is an inertial or relative measurement. In fact, as discussed in [28], in situations where the formation can be grossly distorted such that the baselines between spacecraft are similar in size to the inertial measurements, the EKF 
will converge for a severe level of covariance without the implementation of any robustness enhancement schemes. Similarly, even if it is supposed that for inertial range measurements $\rho_{i j}$ is on the order of 1000's of kilometers (instead of the actual order of $10^{5} \mathrm{~km}$ ), even for a severe level of covariance $b_{h_{k}}$ would typically be at least three orders of magnitude smaller than $\left|\epsilon_{k}^{e k f}\right|$ and $B_{h_{k k}}$ would typically be at least five orders of magnitude smaller than $W_{k k}^{e k f}$; consequently both are negligible. Conversely, since for relative range measurements $\rho_{i j}$ is on the order of 10's of kilometers, for a severe level of covariance $b_{h_{k}}$ and $B_{h_{k k}}$ will typically be of about the same order of magnitude or larger than $\left|\epsilon_{k}^{e k f}\right|$ and $W_{k k}^{e k f}$, respectively, and consequently quite significant.

Thus, the GSF has demonstrated the ability to prevent divergence even if the second-order terms are added only to the relative measurement components in the update equations. However, while doing so can decrease computation costs, this must be done cautiously so as to avoid neglecting any significant off-diagonal terms $B_{h_{j k}}$ that are correlated with the relative measurements.

Conversely, even if it is not practical to implement the GSF, the bounds developed in Eqs. (5.12) and (5.13) can still be used to quantify the overall significance of the quadratic nonlinearities by determining at what covariance level the second-order terms become insignificant enough to allow the introductions of small magnitude range measurements. While the covariance levels denoted "moderate" and "severe" are defined for this case in Table 5.1, in general these two concepts are intended to indicate soft measures of covariance levels such that fusing relative measurements result in filter convergence for the 
former and filter divergence for the latter. The exact region that defines these levels is problem dependent, or more precisely, a function of the magnitude of the range measurement. For example, if instead $\rho_{i j}=0.1 \mathrm{~km}$ is considered, the covariance level definitions in Table 5.1 no longer apply. Moreover, secondorder terms would no longer be adequate to prevent filter divergence for Case 3.1 , as this combination of covariance and range magnitude will likely cause cubic nonlinearities to become significant.

Consequently, instead consider the more aptly defined "critical" covariance, which is defined as the covariance at which point $b_{h_{k}} \approx\left|\epsilon_{k}^{e k f}\right| .^{3}$ Thus, for covariance levels near or larger than the critical covariance the quadratic nonlinearities are significant. Equating $b_{h_{k}}$ and $\left|\epsilon_{k}^{e k f}\right|$ for the relative measurement case $^{4}\left(k>m_{\text {in }}\right)$ of Eq. (5.17) and solving for the $\sigma_{\mathbf{r}}$, the critical covariance can then be defined as the square of

$$
\sigma_{\mathbf{r}}^{c r i t}=\frac{\sqrt{2}}{3} \rho_{i j}
$$

Therefore, in Case 3.1 when $\rho_{i j}=10 \mathrm{~km}$, the critical value of $\sigma_{\mathbf{r}}$ is $4.7 \mathrm{~km}$, which as would be expected, is roughly midway between the moderate and severe levels defined in Table 5.1. Also, this critical covariance results in a

\footnotetext{
${ }^{3}$ The $b_{h_{k}} \approx\left|\epsilon_{k}^{e k f}\right|$ definition was chosen instead of $B_{h_{k k}} \approx W_{k k}^{e k f}$ because as implied in the discussion following Eq. (5.18), as well as in Maybeck [40], the $b_{h_{k}}$ terms are typically much more significant. However, using this alternate definition the resulting critical value would be $\sqrt{2}$ times smaller than that of Eq. (5.19).

${ }^{4}$ The relative measurement case was used because not only is it more likely to cause filter complications, but also because it results in a more conservative value: using the inertial measurement case $\left(1 \leq k \leq m_{i n}\right)$, the resulting critical value would be $\sqrt{2}$ times larger than that of Eq. (5.19).
} 
$\operatorname{trace}\left(\mathbf{P}_{\text {pos }}^{-}\right) \approx 133 \mathrm{~km}^{2}$, which as seen in Figure 5.5, corresponds to a maximum value for $b_{h_{k}}$ that is equal to the expected value for the residual, $\left|\epsilon_{k}^{e k f}\right| \approx 6.7$ $\mathrm{km}$, at that particular covariance level.

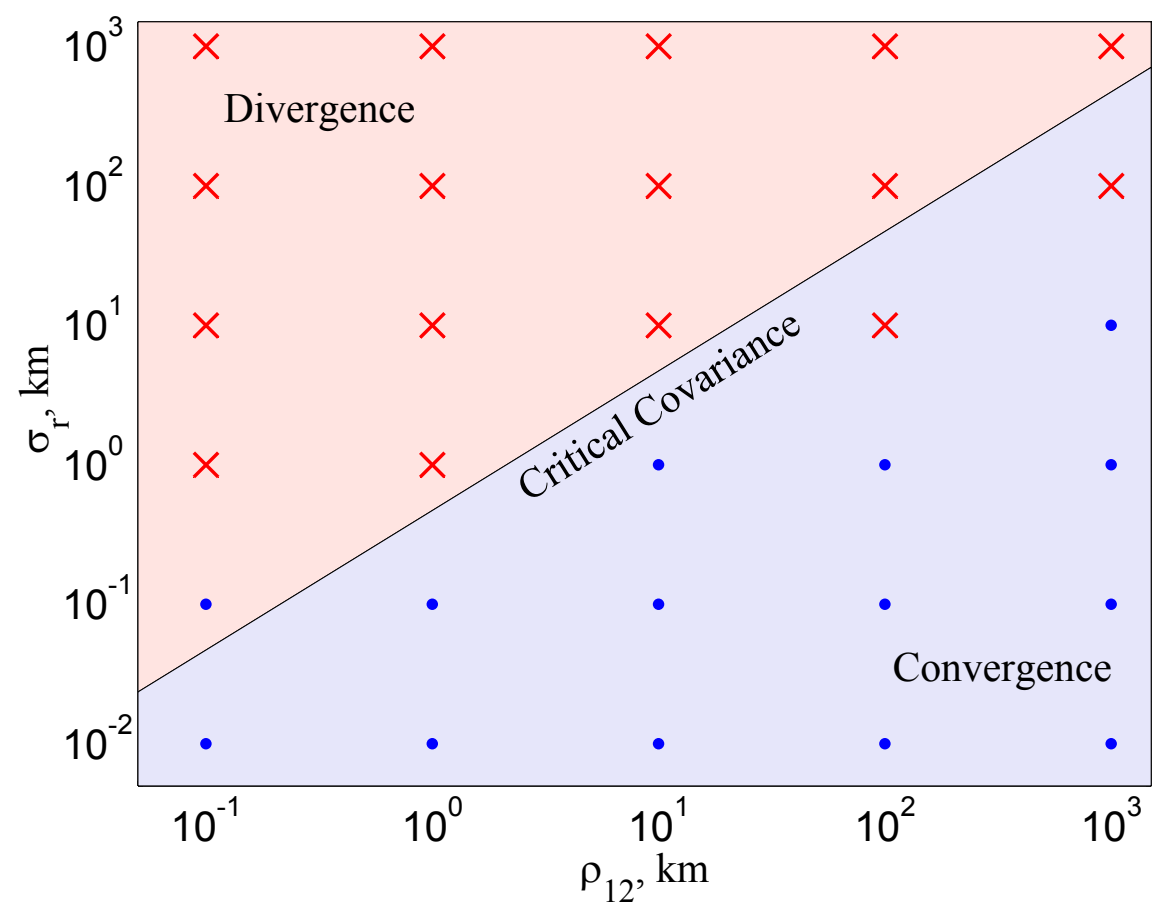

Figure 5.6: Navigation performance for various instances of Case 1.1 with the corresponding critical covariance defined by $\sigma_{\mathbf{r}}^{\text {crit }}$.

To further illustrate this concept the two-spacecraft formation of Case 1.1 was reexamined for various combinations of position covariance levels $\sigma_{\mathbf{r}}^{2}$ (with $\sigma_{\mathbf{v}} \sim 10^{-4} \sigma_{\mathbf{r}}$ ) and relative range magnitudes $\rho_{12}$ (obtained by altering the formation spacing). In Figure 5.6, the blue “•” markers denote instances in which the filter converged, while the red " $x$ " markers denote instances in 
which the filter diverged. As expected, instances in which the covariance is comparable to the critical value are subject to potential fusion complications. Thus, this analysis results in the rule of thumb that range measurements of magnitude $\rho_{i j}$ must be processed with extreme caution when in the presence of position covariance levels on the order of $\sigma_{\mathbf{r}}^{\text {crit }}$.

\subsection{Robustness Enhancement Simulation Results}

Several schemes have been examined to increase filter robustness when fusing inertial and relative range measurements. Table 5.5 summarizes the performance of the following schemes at a final time of $t_{f}=t_{o p}$ : (0) No Scheme for Case 3.0, (I-C) Adaptive Measurement Noise Scheme for Case 3.1, (I-D) Selective Measurement Scheme for Case 3.1, and (II) Second-Order Method for Case 3.1.

Table 5.5: Robustness Enhancement Scheme Performance Comparison

\begin{tabular}{ccccccc}
\hline \hline Scheme & $\begin{array}{c}\bar{P}_{x x}^{1 / 2}\left(t_{f}\right) \\
(\mathrm{m})\end{array}$ & $\begin{array}{c}\bar{P}_{y y}^{1 / 2}\left(t_{f}\right) \\
(\mathrm{m})\end{array}$ & $\begin{array}{c}\bar{P}_{z z}^{1 / 2}\left(t_{f}\right) \\
(\mathrm{m})\end{array}$ & $\begin{array}{c}\bar{P}_{\dot{x} \dot{x}}^{1 / 2}\left(t_{f}\right) \\
(\mathrm{mm} / \mathrm{s})\end{array}$ & $\begin{array}{c}\bar{P}_{\dot{y} \dot{y}}^{1 / 2}\left(t_{f}\right) \\
(\mathrm{mm} / \mathrm{s})\end{array}$ & $\begin{array}{c}\bar{P}_{\dot{z} \dot{z}}^{1 / 2}\left(t_{f}\right) \\
(\mathrm{mm} / \mathrm{s})\end{array}$ \\
\hline 0 & 0.532 & 1.164 & 1.463 & 0.473 & 1.230 & 0.980 \\
I-C & 0.390 & 0.843 & 0.702 & 0.372 & 0.568 & 0.709 \\
I-D & 0.393 & 0.871 & 0.736 & 0.393 & 0.600 & 0.744 \\
II & 0.365 & 0.804 & 0.682 & 0.353 & 0.555 & 0.672 \\
\hline \hline
\end{tabular}

The over bar on the state error covariance $P$ indicates that the values shown are the average values of all four spacecraft. This was done since the state error covariance history of each spacecraft is nearly identical. By successfully fusing the relative measurements using one of the aforementioned 
robustness enhancements schemes, the filter achieves essentially the same increase in navigation as described in Table 4.2 for Case 2. Thus, the navigation state error plots for Case 3.1, Scheme I or II, have not been presented here as they look very similar to what was seen in Figures 4.13 and 4.14. This result is to be expected though since after the covariance level is quickly reduced Case 3 is essentially the same as Case 2. One difference however can be found in

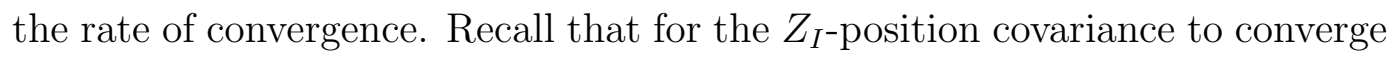
to less than the measurement noise of $5 \mathrm{~m}$, Case 2.1 required only 28 measurement updates. Now, for Case 3.1, Schemes I-C and II required 51 measurement updates and Scheme I-D required 58 measurement updates (recall updates occur every minute). This decrease in convergence rate is because not only is the initial covariance larger, but more importantly, the relative measurements are essentially ignored during the first several updates. 


\section{Chapter 6}

\section{Conclusions}

\subsection{Research Summary}

One contribution of this research is the development of the relationship between observer-formation geometry and navigation performance in Chapter 4 . Section 4.2 begins by examining the relationship between observerformation geometry and system observability. It was demonstrated that certain geometries can lead to a reduction in observability and consequently a decrease in navigation accuracy. Additionally, an inverse analysis was considered to propose formation geometries that may result in an increase in navigation performance. Section 4.3 continued this development by examining the relationship between system observability and the expected navigation accuracy. In particular, the correlation between the state error covariance and the LTI observability, the Cramér-Rao lower bound, and the commonly used DOP metric was demonstrated. Of which, the latter two entailed the deriva-

tion of the explicit relationship (seen in Eq. (4.51)) between the observability Gramian and the Fisher information matrix used in the Cramér-Rao lower bound.

Chapter 5 considered the decrease in the robustness of the EKF re- 
sulting from fusing inertial and relative range measurements. In particular, Section 5.2 examined some common methods used to avoid fusion complications. In Section 5.3 second-order terms that are normally truncated were thoroughly examined and the exact cause of the inertial-relative measurement fusion complications was determined. It was found that although these terms are negligible for large inertial range measurements, they can become quite significant when using small relative range measurements. This is especially true considering that the smaller relative range measurements will typically be more accurate. More specifically however, it turns out that the magnitude of the range measurement poses the potential problem, not the measurement type, be it inertial or relative. Thus, any relatively small magnitude range measurement must be processed with extreme caution when in the presence of state error covariances on the order of the critical covariance as defined in Eq. (5.19).

\subsection{Supplementary Research}

Supplementary work for Section 4.2 included the use of inertial rangerate measurements $\left(\dot{\rho}_{i j}\right)$ and the addition of lunar gravity during the cislunar transfer trajectory (Case 0). Using the framework established in this section, range-rate measurements were found to produce similar geometric anomalies. For example, the corresponding set of rows of the LTI observability matrix is linearly dependent when the inertial range-rate vector is parallel to the inertial range vector. However, unlike what was seen in the range measurement 
case, with the addition of range-rate measurements the nonlinear observability matrix and LTI observability matrix become noticeably different. The addition of lunar gravity terms however, do not have this effect and they can easily be incorporated into Eq. (4.18), by appropriately altering $\mathbf{g}, \mathbf{G}$, and $\mathbf{C}_{k}$.

Additionally, an alternate benchmark mission was also considered. The alternate mission was based on the geocentric highly elliptic orbit (HEO), fourspacecraft formation flying mission presented in Carpenter, et al. [12]. The HEO mission is part of a set of three benchmark missions that were developed to provide focus to the distributed spacecraft system research community, while remaining generic enough to capture a wide range of mission features that will be of interest to NASA over the next couple of decades.

The nonlinear observability matrix was also examined for the HEO mission. However, in order to make the problem manageable, only two spacecraft were considered with a single measurement consisting of the relative range between them. To further reduce the dimension, the spacecraft were placed in coplanar orbits and the LVLH frame was used, centered at one of the spacecraft. The Hill-Clohessy-Wiltshire equations were then re-derived for the elliptical problem (no simplifying circular assumptions) to obtain the equations of motion for the relative dynamics between the spacecraft. Although the resulting nonlinear observability matrix is noticeably different than LTI observability matrix, the observability measures for both do exhibit the same major trends. Furthermore, these trends were discussed in relation to the line of sight angle, and its corresponding angular velocity and angular acceleration. 
Finally, as alluded to in Section 4.2.2, the HEO mission was also considered to illustrate the analysis of Chapter 5. The results, which were analogous, were presented at the 16th AAS/AIAA Spaceflight Mechanics Meeting [28].

\subsection{Future Research Considerations}

The consideration of alternate problem formulations may provide additional insight into the relationship between observer geometry, system observability, and navigation performance. One possible example of this is the analysis of the lunar mission cases using the Hill-Clohessy-Wiltshire equations in the LVLH frame. Such a formulation results in LTI dynamics such that $\dot{\mathbf{x}}=\mathbf{F x}$. Since the individual spacecraft dynamics can easily be uncoupled from the other spacecraft, for simplicity considering here the state dynamics for only one spacecraft results in,

$$
\mathbf{F}=\left[\begin{array}{c|c}
\mathbf{0} & \mathbf{I} \\
\hline \boldsymbol{\Omega}_{\mathbf{r}} & \boldsymbol{\Omega}_{\mathbf{v}}
\end{array}\right], \boldsymbol{\Omega}_{\mathbf{r}}=\left[\begin{array}{ccc}
0 & 0 & 0 \\
0 & -\omega_{*}^{2} & 0 \\
0 & 0 & 3 \omega_{*}^{2}
\end{array}\right], \boldsymbol{\Omega}_{\mathbf{v}}=\left[\begin{array}{ccc}
0 & 0 & 2 \omega_{*} \\
0 & 0 & 0 \\
-2 \omega_{*} & 0 & 0
\end{array}\right]
$$

where $\omega_{*}$ is the angular velocity of the reference point as defined in Eq. (2.4).

Then, since $\mathbf{F}$ is constant, the state transition matrix can be obtained via,

$$
\boldsymbol{\Phi}\left(t_{d}, t_{d-1}\right)=e^{\left(t_{d}-t_{d-1}\right) \mathbf{F}}=\mathscr{L}^{-1}\left[(s \mathbf{I}-\mathbf{F})^{-1}\right]
$$

where $\mathscr{L}^{-1}$ denotes the inverse Laplace transform and here $s$ is the complex variable of the Laplace transform [18]. Evaluating Eq. (6.2), the state transi- 
tion matrix can be computed analytically as,

$$
\boldsymbol{\Phi}=\left[\begin{array}{cccccc}
1 & 0 & 6 \theta_{*}-6 S_{\theta_{*}} & \frac{-3 \theta_{*}+4 S_{\theta_{*}}}{\omega_{*}} & 0 & \frac{2-2 C_{\theta_{*}}}{\omega_{*}} \\
0 & C_{\theta_{*}} & 0 & 0 & \frac{S_{\theta_{*}}}{\omega_{*}} & 0 \\
0 & 0 & 4-3 C_{\theta_{*}} & \frac{-2+2 C_{\theta_{*}}}{\omega_{*}} & 0 & \frac{S_{\theta_{*}}}{\omega_{*}} \\
0 & 0 & 6 \omega_{*}-6 \omega_{*} C_{\theta_{*}} & -3+4 C_{\theta_{*}} & 0 & 2 S_{\theta_{*}} \\
0 & -\omega_{*} S_{\theta_{*}} & 0 & 0 & C_{\theta_{*}} & 0 \\
0 & 0 & 3 \omega_{*} S_{\theta_{*}} & -2 S_{\theta_{*}} & 0 & C_{\theta_{*}}
\end{array}\right]
$$

where the following have been defined for compactness: $\mathbf{\Phi}=\boldsymbol{\Phi}\left(t_{d}, t_{d-1}\right), \theta_{*}:=$ $\omega_{*}\left(t_{d}-t_{d-1}\right)$ (in radians), $S_{\theta_{*}}:=\sin \theta_{*}$, and $C_{\theta_{*}}:=\cos \theta_{*}$. Thus, using Eq. (6.3) to compute the observability Gramian analytically, it may be possible to infer additional information about the observability due the geometry and dynamics of the formation.

Another consideration involves the hypothetical situation in which the inertial frame is the most convenient choice of coordinate systems, yet the driving requirement of the navigation performance is the accuracy of the relative state. In the derivation of the discrete Kalman filter, the optimum choice for the Kalman gain $\mathbf{K}$ (seen in Eq. (3.32)) is obtained by minimizing the performance index $J\left(t_{d}\right)=\operatorname{trace}\left(\mathbf{P}\left(t_{d}^{+}\right)\right.$), which (for an unbiased estimate) is essentially equivalent to minimizing the inertial state error. Since this does not necessarily imply that the relative state errors are minimized, it may be of interest to rederive the Kalman gain equation using a more appropriate performance index. For example, if underlying goal is to minimize the relative position errors between Spacecraft $i$ and Spacecraft $j$, the performance index 
$J\left(t_{d}\right)=\operatorname{trace}\left(\mathbf{P}_{\mathbf{r}_{i} \mathbf{r}_{j}}^{r e l}\left(t_{d}^{+}\right)\right)$where $\mathbf{P}_{\mathbf{r}_{i} \mathbf{r}_{j}}^{r e l}$ is defined in Eq. (5.3), may offer superior navigation performance.

These are just two examples of how the navigation observability analysis could be expanded. In general the type of analysis presented in this dissertation is quite open-ended and may require ad hoc methods for definitive answers. Nonetheless this work has provided basic methodologies and rules-of-thumb to aid in the navigation design process for orbital applications in formation flying. 


\section{Bibliography}

[1] P. A. M. Abusali, B. D. Tapley, and B. E. Schutz. Autonomous Navigation of Global Positioning System Satellites Using Cross-Link Measurements. Journal of Guidance, Control, and Dynamics, 21(2):321-327, March-April 1998.

[2] National Aeronautics and Space Administration. http://liftoff.msfc. nasa.gov/academy/rocket_sci/shuttle/coord/in_lv.gif.

[3] Roger R. Bate, Donald D. Mueller, and Jerry E. White. Fundamentals of Astrodynamics. Dover Publications, Inc., New York, NY, 1971.

[4] Robert H. Bishop, P. Daniel Burkhart, and Sam Thurman. Observability During Planetary Approach Navigation. Proceedings of the AAS/AIAA Spaceflight Mechanics Meeting, February 1993.

[5] Robert H. Bishop and Nassib Nabaa. Development of a Practical Multitarget Multisensor Tracking System. Technical report, Oerlikon-Contraves AG, Zrich, Switzerland, March 1997.

[6] Charles D. Brown. Spacecraft Mission Design. American Institute of Aeronautics and Astronautics, Inc., Reston, VA, 1998. 
[7] Robert Grover Brown and Patrick Y. C. Hwang. Introduction to Random Signals and Applied Kalman Filtering. John Wiley and Sons, Inc., New York, NY, 3rd edition, 1997.

[8] D. S. Burnett, B. L. Barraclough, R. Bennett, M. Neugebauer, L. P. Oldham, C. N. Sasaki, D. Sevilla, N. Smith, E. Stansbery, D. Sweetnam, and R. C. Wiens. The Genesis Discovery Mission: Return of Solar Matter to Earth. Space Science Reviews, 105(3-4):509-534, 2003.

[9] Franz D. Busse, Jonathan P. How, and James Simpson. Demonstration of Adaptive Extended Kalman Filter Low-Earth-Orbit Formation Estimation Using CDGPS. Journal of The Institute of Navigation, 50(2):79-93, Summer 2003.

[10] J. P. Le Cadre and C. Jauffret. Discrete-Time Observability and Estimability Analysis for Bearings-Only Target Motion Analysis. IEEE Transactions on Aerospace and Electronic Systems, 33(1):178-201, January 1997.

[11] J. Russell Carpenter and Kyle T. Alfriend. Navigation Accuracy Guidelines for Orbital Formation Flying. Proceedings of the AIAA Guidance, Navigation, and Control Conference, August 2003.

[12] J. Russell Carpenter, Jesse A. Leitner, David C. Folta, and Richard D. Burns. Benchmark Problems for Space Mission Formation Flying. Proceedings of the AIAA Guidance, Navigation, and Control Conference, August 2003. 
[13] Chi-Tsong Chen. Linear System Theory and Design. Oxford University Press, New York, NY, 3rd edition, 1999.

[14] John L. Crassidis and John L. Junkins. Optimal Estimation of Dynamic Systems. CRC Press, New York, NY, 2004.

[15] R. H. Deaves. Covariance Bounds for Augmented State Kalman Filter Application. Electronic Letters, 35(23):2062-2063, November 1999.

[16] Bernard Delyon. A Note on Uniform Observability. IEEE Transactions on Automatic Control, 46(8):1326-1327, August 2001.

[17] Walter F. Denham and Samuel Pines. Sequential Estimation When Measurement Function Nonlinearity Is Comparable to Measurement Error. AIAA Journal, 4(6):1071-1076, June 1966.

[18] Richard C. Dorf and Robert H. Bishop. Modern Control Systems. Pearson Prentice Hall, Upper Saddle River, NJ, 10th edition, 2005.

[19] Arthur Gelb. Applied Optimal Estimation. MIT Press, Cambridge, MA, 1974 .

[20] Jr. George B. Thomas and Ross L. Finney. Calculus and Analytic Geometry. Addison Wesley, Reading, MA, 8th edition, 1994.

[21] Gene H. Golub and Charles F. Van Loan. Matrix Computations. The Johns Hopkins University Press, Baltimore, MD, 3rd edition, 1996. 
[22] E. W. Griffith and K. S. P. Kumar. On the Observability of Nonlinear Systems: I. Journal of Mathematical Analysis and Applications, 35(1):135-147, July 1971.

[23] Fredric M. Ham and R. Grover Brown. Observability, Eigenvalues, and Kalman Filtering. IEEE Transactions on Aerospace and Electronic Systems, AES-19(2):269-273, March 1983.

[24] Robert Hermann and Arthur J. Krener. Nonlinear Controllability and Observability. IEEE Transactions on Automatic Control, AC-22(5):728740, October 1977.

[25] Roger A. Horn and Charles R. Johnson. Matrix Analysis. Cambridge University Press, Cambridge, MA, 1985.

[26] D. G. Hull, J. L. Speyer, and D. B. Burris. Linear-Quadratic Guidance Law for Dual Control of Homing Missiles. Journal of Guidance, Control, and Dynamics, 13(1):137-144, January-February 1990.

[27] Paul J. Huxel and Robert H. Bishop. Navigation Algorithms for Formation Flying Missions. Proceedings of the 2nd International Symposium on Formation Flying Missions and Technologies, September 2004.

[28] Paul J. Huxel and Robert H. Bishop. Fusing Inertial and Relative Measurements for Inertial Navigation in the Presence of Large State Error Covariances. Proceedings of the 16th AAS/AIAA Spaceflight Mechanics Meeting, January 2006. 
[29] Alberto Isidori. Nonlinear Control Systems. Springer-Verlag, New York, NY, 3rd edition, 1995.

[30] Rudolph E. Kalman. A New Approach to Linear Filtering and Prediction Problems. Transactions of the ASMEJournal of Basic Engineering, 82(Series D):35-45, 1960.

[31] Rudolph E. Kalman. Contributions on the Theory of Optimal Control. Boletin de la Sociedad Matematica Mexicana, 5:102-119, 1960.

[32] Rudolph E. Kalman. On the General Theory of Control Systems. Proceedings of the 1st IFAC Congress, 1:481-492, 1960.

[33] David Kelbel, Taesul Lee, Anne Long, Russell Carpenter, and Cheryl Gramling. Relative Navigation Algorithms for the MMS Formation. NASA Flight Mechanics Symposium, October 2003.

[34] Arthur J. Krener and Wei Kang. Locally Convergent Nonlinear Observers. SIAM Journal on Control and Optimization, 42(1):155-177, 2003.

[35] David C. Lay. Linear Algebra and Its Applications. Addison Wesley, Boston, MA, 3rd edition, 2003.

[36] Don Lerro and Yaakov Bar-Shalom. Tracking With Debiased Consistent Converted Measurements Versus EKF. IEEE Transactions on Aerospace and Electronic Systems, 29(3):1015-1022, July 1993. 
[37] L. Lin, T. Kirubarajan, and Y. Bar-Shalom. 3-D Track Initiation in Clutter Using 2-D Radar Measurements. IEEE Transactions on Aerospace and Electronic Systems, 38(4):1434-1441, October 2002.

[38] Anne Long, Dominic Leung, David Kelbel, Mark Beckman, and Cheryl Gramling. Navigation Concepts for the James Webb Space Telescope. NASA Flight Mechanics Symposium, October 2005.

[39] Peter S. Maybeck. Stochastic Models, Estimation, and Control, volume 1. Academic Press, New York, NY, 1979.

[40] Peter S. Maybeck. Stochastic Models, Estimation, and Control, volume 2. Academic Press, New York, NY, 1982.

[41] Nassib Nabaa and Robert H. Bishop. Clustering Approach to the Multitarget Multisensor Tracking Problem. Proceedings of the SPIE - The International Society for Optical Engineering, 3136:226-237, July 1997.

[42] Yaakov Oshman and Pavel Davidson. Optimization of Observer Trajectories for Bearings-Only Target Localization. IEEE Transactions on Aerospace and Electronic Systems, 35(3):892-902, July 1999.

[43] M. Pachter and T. Q. Nguyen. An Efficient GPS Position Determination Algorithm. Journal of the Institute of Navigation, 50(2):131-141, Summer 2003. 
[44] Seong-Taek Park and Jang Gyu Lee. Design of a Practical Tracking Algorithm with Radar Measurements. IEEE Transactions on Aerospace and Electronic Systems, 34(4):1337-1344, October 1998.

[45] Andre C.M. Ran and Martine C.B. Reurings. A Fixed Point Theorem in Partially Ordered Sets and Some Applications to Matrix Equations. Proceedings of the American Mathematical Society, 132(5):14351443, September 2003.

[46] Obed Sands, Joseph Connolly, Bryan Welch, James Carpenter, Todd Ely, and Kevin Berry. Dilution of Precision-Based Lunar Navigation Assessment for Dynamic Position Fixing. Proceedings of the ION National Technical Meeting, January 2006.

[47] F. H. Schlee, C. J. Standish, and N. F. Toda. Divergence in the Kalman Filter. AIAA Journal, 5(6):1114-1120, June 1967.

[48] Alan M. Schneider. Observability of Relative Navigation Using RangeOnly Measurements. IEEE Transactions on Aerospace and Electronic Systems, AES-21(4):569-581, July 1985.

[49] Taek Lyul Song. Observability of Target Tracking with Range-Only Measurements. IEEE Journal of Oceanic Engineering, 24(3):383-387, July 1999.

[50] Victor Szebehely. Theory of Orbits (The Restricted Problem of Three Bodies). Academic Press, New York, NY, 1967. 
[51] James H. Taylor. The Cramér-Rao Estimation Error Lower Bound Computation for Deterministic Nonlinear Systems. IEEE Transactions on Automatic Control, AC-24(2):343-344, April 1979.

[52] N. F. Toda, F. H. Schlee, and P. Obsharsky. Region of Kalman Filter Convergence for Several Autonomous Navigation Modes. AIAA Journal, 7(4):622-627, April 1969.

[53] Harry L. Van Trees. Detection, Estimation, and Modulation Theory. John Wiley and Sons, Inc., New York, NY, 1968.

[54] David A. Vallado. Fundamentals of Astrodynamics and Applications. The McGraw-Hill Companies, Inc., New York, NY, 1997.

[55] William S. Widnall. Enlarging the Region of Convergence of Kalman Filters Employing Range Measurements. AIAA Journal, 11(3):283-287, March 1973.

[56] Jijie Zhu. Calculation of Geometric Dilution of Precision. IEEE Transactions on Aerospace and Electronic Systems, 28(3):893-5, July 1992. 


\section{Vita}

Paul John Huxel was born in Chicago, Illinois on November 20, 1977, the son of John E. Huxel and Jacqueline A. Huxel. He began his aerospace engineering studies in the fall of 1995 when he enrolled in The University of Colorado at Boulder. Paul graduated with his Bachelor of Science degree in May of 1999 and his Master of Science degree in May of 2000. The following fall he entered the graduate aerospace engineering program at The University of Texas at Austin. After two years with the university's Center for Space Research he began work with Dr. Bishop in the fall of 2002. Upon completion of his doctoral studies, Paul will begin work at Draper Laboratory in Cambridge, Massachusetts.

Permanent address: 2917 N. 73rd Avenue

Elmwood Park, Illinois 60707

This dissertation was typeset with $\mathrm{HT}_{\mathrm{E}} \mathrm{X}^{\dagger}$ by the author.

\footnotetext{
${ }^{\dagger} \mathrm{LT}_{\mathrm{E}} \mathrm{X}$ is a document preparation system developed by Leslie Lamport as a special version of Donald Knuth's $\mathrm{T}_{\mathrm{E}} \mathrm{X}$ Program.
} 